\title{
Taxonomic revision of the Neotropical spiders of the genus Idiops Perty, 1833 (Araneae, Idiopidae), with description of four new species
}

\author{
Rafael FONSECA-FERREIRA ${ }^{1, *}$, José Paulo Leite GUADANUCCI ${ }^{2}$, \\ Flávio Uemori YAMAMOTO ${ }^{3} \&$ Antonio Domingos BRESCOVIT ${ }^{4}$ \\ ${ }^{1}$ Pós-graduação em Ecologia, Evolução e Biodiversidade, Instituto de Biociências, \\ Universidade Estadual Paulista, Avenida 24A 1515, 13506-900 Rio Claro-SP, Brazil. \\ ${ }^{2}$ Departamento de Ecologia, Evolução e Biodiversidade, Instituto de Biociências, \\ Universidade Estadual Paulista, Avenida 24A 1515, 13506-900 Rio Claro-SP, Brazil. \\ ${ }^{3,4}$ Laboratório de Coleções Zoológicas, Instituto Butantan, Av. Vital Brasil, 1500, \\ Butantã, São Paulo, São Paulo, CEP 05503-900, Brazil. \\ "Corresponding author: rafaelbioferreira@gmail.com \\ 2Email: jose.guadanucci@unesp.br \\ ${ }^{3}$ Email: flayamamoto@gmail.com \\ [4Email: antonio.brescovit@butantan.gov.br

\footnotetext{
${ }^{1}$ urn:lsid:zoobank.org:author:8E23FE6D-3D20-4667-9F69-15B132D9537B

${ }^{2}$ urn:lsid:zoobank.org:author:D4955FF5-FE7F-4E68-AB2F-4A89593F9850

${ }^{3}$ urn:lsid:zoobank.org:author:86719D83-2F02-4122-8CCE-D0932A3206D0
} \\ ${ }^{4}$ urn:1sid:zoobank.org:author:D5B81D79-AFAE-47B1-8A6E-DAB448A24BCC
}

\begin{abstract}
Neotropical species of the genus Idiops Perty, 1833 are reviewed, and four new species are described from Brazil: I. duocordibus Fonseca-Ferreira, Guadanucci \& Brescovit sp. nov., I. guri FonsecaFerreira, Guadanucci \& Brescovit sp. nov., I. mocambo Fonseca-Ferreira, Guadanucci \& Brescovit sp. nov. and I. sertania Fonseca-Ferreira, Guadanucci \& Brescovit sp. nov. The majority of species are redescribed based on the examination of the types and extensive material. Males of I. petiti (Guérin, 1838), I. rastratus (Pickard-Cambrige, 1889), I. rohdei Karsch, 1886 and I. nilopolensis Mello-Leitão, 1923, and females of I. fuscus Perty, 1833 and I. pirassununguensis Fukami \& Lucas, 2005, hitherto unknown, are described for the first time. Idiops nilopolensis, considered a nomen dubium, is revalidated. Idiops fulvipes Simon, 1889 is synonymized with I. argus Simon, 1889, and I. santaremius (Pickard-Cambrige, 1896) is synonymized with I. petiti. Neotypes are designated for Idiops fuscus, I. nilopolensis and I. siolii (Bücherl, 1953). Idiops bonapartei Hasselt, 1888 is considered species inquirendae, since the type is an immature female. Finally, an updated distribution map of Neotropical species is included. The genus now has 24 species in the Neotropical region.
\end{abstract}

Keywords. Mygalomorphae, Idiopinae, Domiothelina, Arachnida, trapdoor spiders. 
Fonseca-Ferreira R., Guadanucci J.P.L., Yamamoto F.U. \& Brescovit A.D. 2021. Taxonomic revision of the Neotropical spiders of the genus Idiops Perty, 1833 (Araneae, Idiopidae), with description of four new species. European Journal of Taxonomy 780: 1-71. https://doi.org/10.5852/ejt.2021.780.1581

\section{Introduction}

The family Idiopidae Simon, 1889 comprises species popularly known as spiny trapdoor spiders. The spiders build and live in silk-lined burrows in the soil, which are closed with a camouflaged operculum, which functions as a trapdoor (Raven 1985; Dippenaar-Schoeman 2002; Fig. 1A-L). Idiopidae was erected by Raven (1985), who raised the tribe Idiopiae Simon, 1889, until then included in Ctenizidae Thorell, 1887, to the family level. Sustained by three apparently apomorphic features of the male palp (Raven 1985), the family monophyly was never questioned, being well supported by both morphological (Goloboff 1993) and molecular data (Hedin \& Bond 2006; Bond et al. 2012; Opatova et al. 2020). With an origin dating back to the Lower Cretaceous, about 133 million years ago (Opatova et. al. 2020), and Gondwanan distribution (World Spider Catalog 2021), the family Idiopidae is currently divided into three subfamilies: Arbanitinae Simon, 1903, with spiders restricted to Oceania, Genysinae Simon, 1903, occurring in India, Sri Lanka, Madagascar and South America, and Idiopinae Simon, 1889, from South America, Africa, the Middle East, and Southern and Southeast Asia (Raven 1985; World Spider Catalog 2021). The idiopids are currently the third most diverse mygalomorph family, comprising 434 species in 23 genera (World Spider Catalog 2021). Despite this potential, only genera belonging to the subfamily Arbanitinae have recently been systematically reviewed, which have also formed the basis of subsequent biogeographic studies (Rix et al. 2017a, 2017b; Wilson et al. 2018, 2020).

Representatives of the subfamily Idiopinae are characterized by having the anterior lateral eyes projected in front of the others, close to the anterior margin of the carapace, the cephalic area of the carapace arched, distal segments of the front legs with numerous lateral spines and the tibia of leg I of males with the tibial apophysis with one or two apical branches (Raven 1985). It currently has seven genera: Ctenolophus Purcell, 1904, Galeosoma Purcell, 1903, Gorgyrela Purcell, 1902, Segregara Tucker, 1917 and Titanidiops Simon, 1903, restricted to the African continent, Heligmomerus Simon, 1892 occurring in India, Sri Lanka and Africa, and Idiops Perty, 1833 occurring in South America, Africa, Syria, Israel, Yemen, India, Myanmar and Thailand (World Spider Catalog 2021).

Among the genera of Idiopidae, the most diverse is Idiops Perty, 1833 (Fig. 2A-F), which currently includes 99 valid species (World Spider Catalog 2021). Their body size ranges from 5 to $35 \mathrm{~mm}$ and species are mainly characterized by chelicera with two rows of teeth on the prolateral and retrolateral margins, a sternum with two pairs of sigilla (posterior pair absent) and the absence of tiny spines on the coxae (Raven 1985; Dippenaar-Schoeman 2002). In the cladistic analysis carried out by Raven (1985), Idiops is closely related to Ctenolophus and Galeosoma, grouped in a trichotomy and supported by the absence of the posterior pair of sigillae (Raven 1985). According to the molecular phylogeny proposed by Opatova et al. (2020), Idiops is recovered as the sister-group to four other genera of Idiopinae that were recovered as a monophyletic clade.

Four genera are considered junior synonyms of Idiops. Acanthodon Guérin, 1838, proposed based on the species A. petiti Guérin, 1838 from Brazil, was synonymized with Idiops by Pickard-Cambridge (1870). However, Simon (1892) revalidated Acanthodon, differentiating it from Idiops by its ocular formation and restricted the American species to the genera Pseudidiops Simon, 1889 and Idiops. Later, Simon (1903), when examining a female of Idiops germaini Simon, 1892, considered that the differences in the eyes between Idiops and Acanthodon would not be valid, since they are dependent on sex. Consequently, he synonymized these two genera again, but kept the Idiops genus only for American species. The problem of Acanthodon was only solved by Tucker (1917) and its consequent synonymy with Idiops. Dendricon 

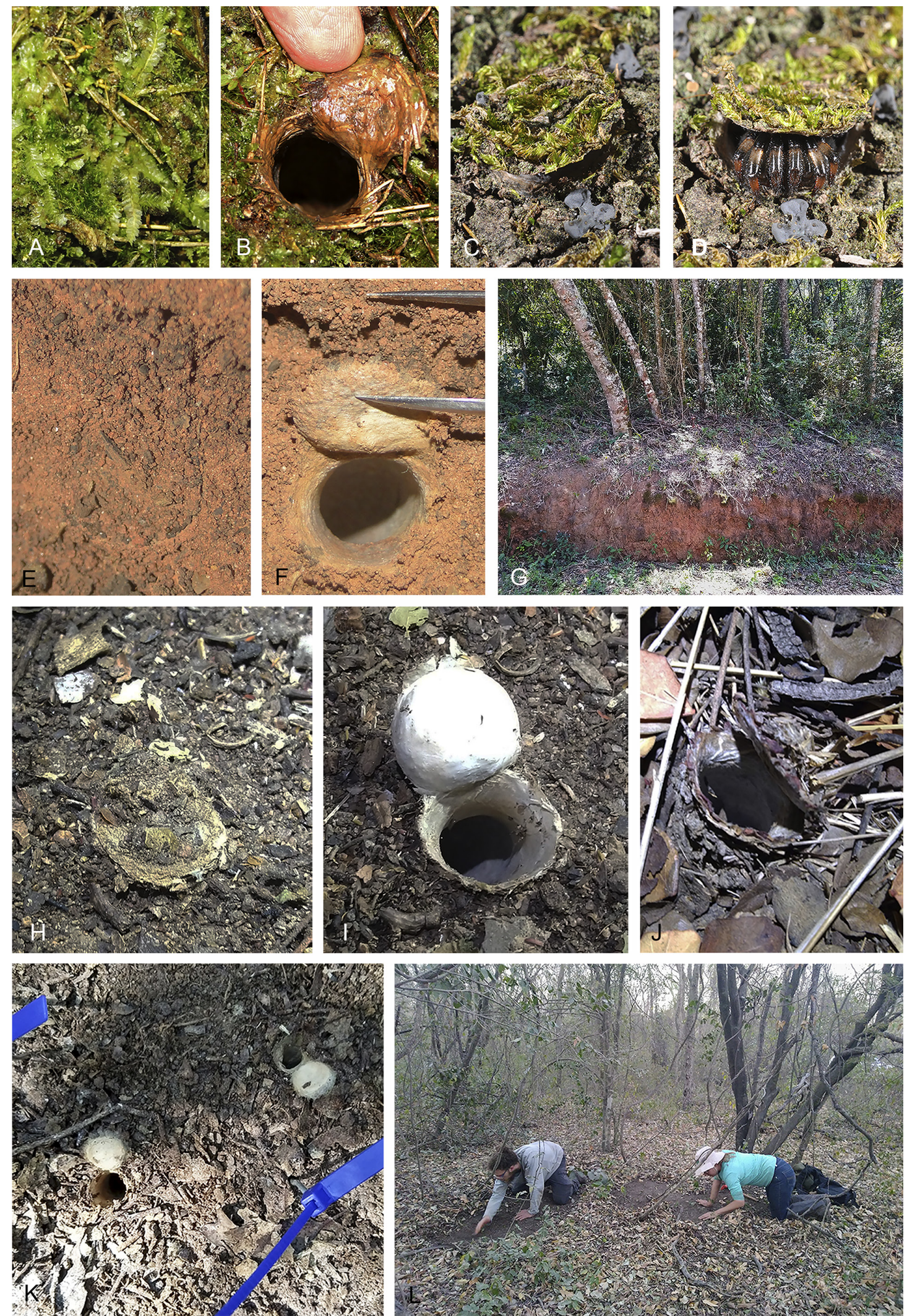

Fig. 1. Natural habitats and diversity of burrows and trapdoors of Idiops Perty, 1833 spp. A-G. Ravine habitats. H-L. Ground habitats. Images by Rafael P. Indicatti (A-B); by Arthur Anker and Pedro H. Martins (C-D); by Leonardo S. Carvalho (E-F); by Rafael Fonseca-Ferreira (G-L). 

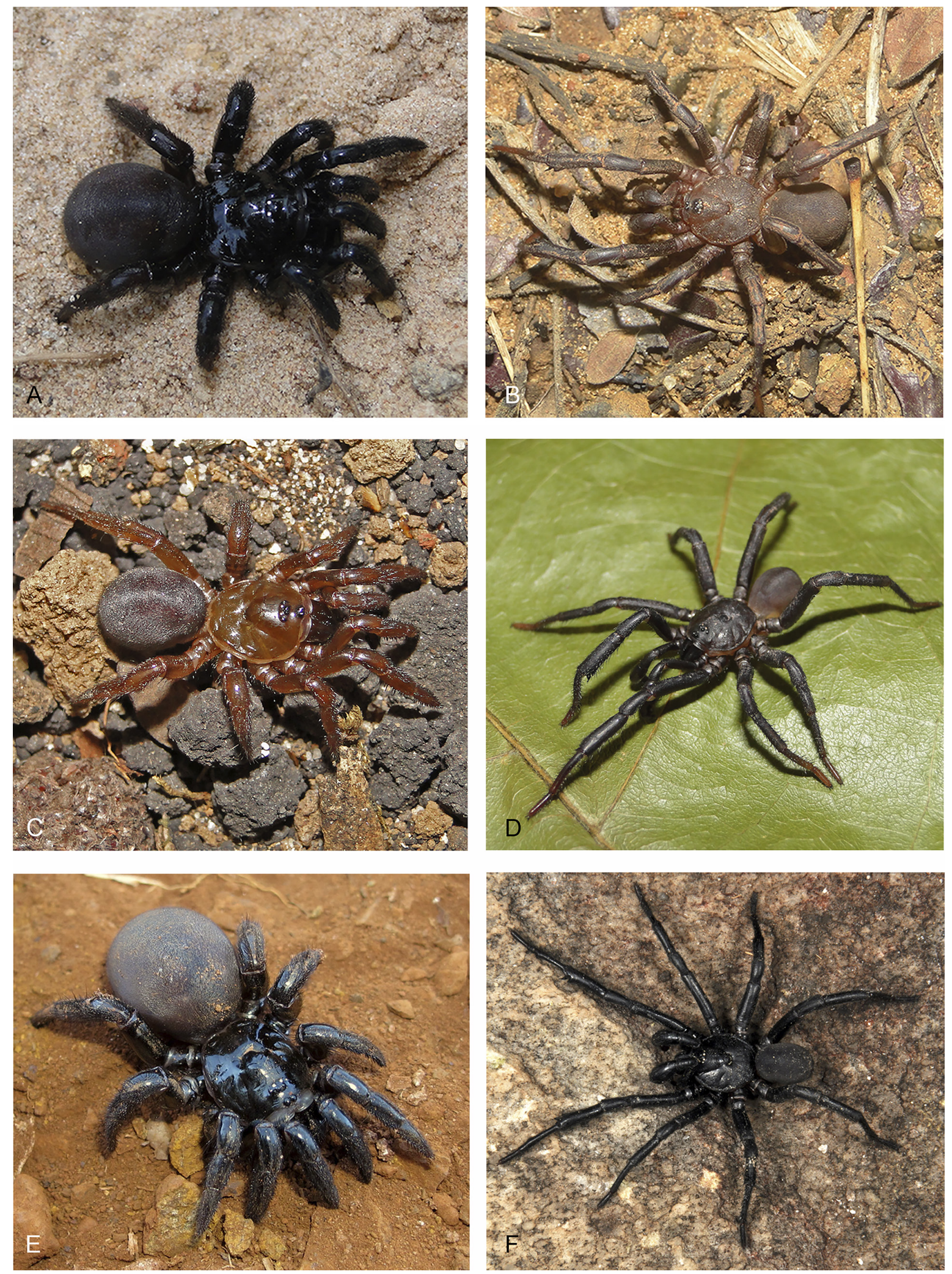

Fig. 2. Live habitus of Idiops Perty, 1833. A. I. fuscus Perty, 1833, ๆ. B. I. fuscus, Ô. C. I. camelus (Mello-Leitão, 1937), ․ D. I. camelus, Ô. E. I. pirassununguensis Fukami \& Lucas, 2005, ㅇ. F. I. pirassununguensis, Ô. Images by: Leonardo S. Carvalho (A-B); Rafael P. Indicatti (C-D); Pedro H. Martins (E-F). 
O. Pickard-Cambridge, 1889 was proposed based on D. rastratum O. Pickard-Cambridge, 1889, but was synonymized with Pseudidiops Simon, 1889 by Pocock (1895). Pseudidiops was proposed based on the species $P$. opifex Pocock 1899, from Colombia. This genus was synonymized by Raven (1985) who considered the characteristics presented by Simon insufficient to differentiate this genus. Juambeltzia Mello-Leitão, 1946 was proposed based on the species J. clara Mello-Leitão, 1946, from Argentina, and later synonymized by Schiapelli \& Gerschman de Pikelin (1971). Pachyidiops Simon, 1903 was established based on the species Idiops crassus Simon, 1884, from Burma and was synonymized by Raven (1985).

So far, no comprehensive review of the genus Idiops has been carried out, only propositions of species (Fonseca-Ferreira et al. 2017; Siliwal et al. 2020) or reviews limited to countries (Fukami \& Lucas 2005; Mirza et al. 2012; Ferretti et al. 2017). Here we present a taxonomic revision of the Neotropical species of Idiops and present an updated distribution map of Neotropical species, thus expanding the knowledge of the family Idiopidae.

\section{Material and methods}

\section{Repositories}

The examined specimens are deposited in the following taxonomic collections (curators indicated between parentheses):

BMNH $=$ The Natural History Museum, London, UK (J. Beccaloni)

CAD $=$ Coleção Aracnológica Diamantina, UNESP, Rio Claro, Brazil (J.P. Guadanucci)

CHNUFPI $=$ Coleção de História Natural, Universidade Federal do Piauí, Brazil (L.S. Carvalho)

FCE $\quad=$ Facultad de Ciencias, Universidad de la República, Montevideo, Uruguay (M. Simó)

IBNP $=$ Inventario Biológico Nacional de Paraguay, Asunción, Paraguay (J.A. Kochalka)

IBSP $=$ Instituto Butantan, São Paulo, Brazil (A.D. Brescovit)

INPA $=$ Instituto Nacional de Pesquisas da Amazônia, Manaus, Brazil (M.L. Oliveira)

$\mathrm{MCN}=$ Museu de Ciências Naturais, Sema, Porto Alegre, Brazil (R. Ott)

MCTP $=$ Museu de Ciências e Tecnologia, Pontifícia Universidade Católica, Porto Alegre, Brazil (R.A. Teixeira)

MHNM $=$ Museo Nacional de História Natural, Montevideo, Uruguay (A. Laborda)

$\mathrm{MNHN}=$ Muséum national d'histoire naturelle, Paris, France (C. Rollard)

MNRJ $=$ Museu Nacional, Universidade Federal do Rio de Janeiro, Rio de Janeiro, Brazil (A.B. Kury)

MPEG $=$ Museu Paraense Emilio Goeldi, Belém, Brazil (A.B. Bonaldo)

MZSP $=$ Museu de Zoologia, Universidade de São Paulo, São Paulo, Brazil (R. Pinto da Rocha)

UFMG $=$ Coleção de Invertebrados, Universidade Federal de Minas Gerais, Belo Horizonte, Brazil (A.J. Santos)

UFMT $=$ Coleção de Arachnida, Universidade Federal do Mato Grosso, Cuiabá, Brazil (A. Chagas Junior)

UFPB = Coleção de Arachnida, Universidade Federal da Paraíba, João Pessoa, Brazil (M.B. da Silva)

ZMB $=$ Zoologisches Museum Berlin, Berlin, Germany (J. Dunlop)

\section{Measurements}

Measurements were taken with the aid of a Leica M205C stereo microscope equipped with a Leica DFC295 digital camera. The measurements of the carapace, abdomen and legs were taken in dorsal view. For the diameter of the eyes, the largest values were considered. Leg measurements are given in the following order: total length (femur, patella, tibia, metatarsus, tarsus). All measurements are in millimeters. The coding and position of the leg spines follow the methodology proposed by Petrunkevitch 
(1925). Spermathecae were dissected and immersed in enzyme (Ultrazyme $\AA$ ) for 24 hours for soft tissue digestion to allow observation of the internal structures.

\section{Illustrations}

Photographs were taken with a Leica DFC295 digital camera, with focus stacking using Leica Application ver. 2.5.0. For scanning electron microscopy (SEM) micrographs, body parts were dehydrated in a series of graded ethanol washes $(80 \%$ to $100 \%)$, dried by critical point, mounted on metal stubs using adhesive copper tape and nail polish for fixation and covered with gold. SEM micrographs were taken with an FEI Quanta 250 scanning electron microscope at the Laboratório de Biologia Celular of Instituto Butantan, São Paulo, Brazil. The drawings were made with the aid of a Leica MZ12.5 stereo microscope equipped with a camera lucida. The final art of the drawings was made with graphite, on $180 \mathrm{~g}$ opaline paper. Illustrations were digitalized using the HP Scanjet G4050 scanner. All generated pictures were later edited in Photoshop CC 2018 software.

\section{Geographical data}

Each locality from museum databases was carefully checked (lat-long coordinates) to correct for imprecise georeferences in decimal degrees, based on the WGS 1984 datum. In the case of samples with inaccurate or incomplete distribution data, the specimens were georeferenced to the geographic center of the given locality. For species from Argentina, recently reviewed by Ferretti et al. 2017, the coordinates available in the material examined were included. Species distribution maps were made using the QGIS ver. 3.12.3 program. Subsequently, they were organized into plates using the Photoshop CC 2018 software.

$\begin{array}{ll}\text { Abbreviations } \\ \mathrm{ALE}=\text { anterior lateral eyes } \\ \mathrm{AME}=\text { anterior median eyes } \\ \mathrm{CL}=\text { carapace length } \\ \mathrm{CW} & =\text { carapace width } \\ \mathrm{d} & =\text { dorsal } \\ \mathrm{DET} & =\text { distal embolar tooth } \\ \mathrm{EL} & =\text { embolar lamella } \\ \mathrm{Fe} & =\text { femur } \\ \mathrm{K} & =\text { keel } \\ \mathrm{Mt} & =\text { metatarsus } \\ \mathrm{LL} & =\text { labium length } \\ \mathrm{LW} & =\text { labium width } \\ \mathrm{p} & =\text { prolateral } \\ \mathrm{Pa} & =\text { patella } \\ \mathrm{PLE} & =\text { posterior lateral eyes } \\ \mathrm{PME} & =\text { posterior median eyes } \\ \mathrm{PT} & =\text { promarginal teeth } \\ \mathrm{r} & =\text { retrolateral } \\ \mathrm{RT} & =\text { retromarginal teeth } \\ \mathrm{SL} & =\text { sternum length } \\ \mathrm{SW} & =\text { sternum width } \\ \mathrm{Ta} & =\text { tarsus } \\ \mathrm{TBL} & =\text { total body length } \\ \mathrm{Ti} & =\text { tibia } \\ \mathrm{V} & =\text { ventral } \\ & \end{array}$




\title{
Results
}

Taxonomy

\author{
Class Arachnida Cuvier, 1812 \\ Order Araneae Clerck, 1757 \\ Family Idiopidae Simon, 1889 \\ Subfamily Idiopinae Simon, 1889
}

Genus Idiops Perty, 1833

Idiops Perty, 1833: 197 (type species by monotypy: I. fuscus Perty, 1833).

Acanthodon Guérin, 1838: 163 (type species by monotypy: A. petitii Guérin, 1838). Synonymized by O. Pickard-Cambridge 1870: 107.

Dendricon O. Pickard-Cambridge, 1889: 250 (type species by monotypy: D. rastratum O. PickardCambridge, 1889). Synonymized by Pocock 1895: 223.

Pseudidiops Simon, 1889: 215 (type species by monotypy: P. opifex Simon, 1889). Synonymized by Raven 1985: 139.

Juambeltzia Mello-Leitão, 1946: 6 (type species by monotypy: J. clara Mello-Leitão, 1946). Synonymized by Schiapelli \& Gerschman de Pikelin 1971: 58.

Idiops - O. Pickard-Cambridge 1870: 101. — Simon 1892: 89. — Roewer 1953: 5. - Raven 1985: 139. - Dippenaar-Schoeman 2002: 68.

Acanthodon - Walckenaer 1837: 434. — Simon 1892: 90; 1903: 350. — Pocock 1897: 731.

\section{Type species}

Idiops fuscus Perty, 1833

\section{Composition}

Idiops includes 102 species, of which 24 occur in the Neotropical region, including the four new species herein described: I. fuscus Perty, 1833, the type species, I. argus Simon, 1889, I. bonapartei Hasselt, 1888, I. cambridgei Ausserer, 1875, I. camelus (Mello Leitão, 1937), I. carajas Fonseca-Ferreira, Zampaulo \& Guadanucci, 2017, I. clarus (Mello-Leitão, 1946), I. duocordibus sp. nov., I. germaini Simon, 1892, I. guri sp. nov., I. harti (Pocock, 1893), I. hirsutipedis Mello-Leitão, 1941, I. minguito Ferretti, 2017, I. mocambo sp. nov., I. nilopolensis Mello-Leitão, 1923, I. opifex (Simon, 1889), I. petiti (Guérin, 1838), I. piluso Ferretti, Nime \& Mattoni, 2017, I. pirassununguensis Fukami \& Lucas, 2005, I. sertania sp. nov., I. siolii (Bücherl, 1953), I. rastratus (O. Pickard-Cambridge, 1889), I. rohdei Karsch, 1886 and I. tolengo Ferretti, 2017.

\section{Distribution}

South America, Africa, Syria, Israel, Yemen, India, Myanmar and Thailand. Here we present the updated distribution of the Neotropical species of the genus (Figs 3-4).

\section{Diagnosis}

The species of Idiops differ from all other Idiopinae by having the coxae without spines and by the chelicerae with a row of large teeth along the retromargin and small teeth concentrated in the basal third of the promargin and arranged in a row or randomly (Fig. 5A-B; except I. harti (Pocock, 1893)). Idiops harti differs from all other species of the genus by having only one prolateral row of large teeth (Fig. 5C). 


\section{Description}

Small to medium sized spiders, with Neotropical species with total length ranging between $5.4 \mathrm{~mm}$ and $19.1 \mathrm{~mm}$. Brown color, with variation between yellowish brown and almost entirely black (Fig. 2A-F). Anterior lateral eyes close to clypeal edge (Fig. 6A). Chelicerae with a prolateral row of large teeth and a retrolateral row of smaller teeth (Fig. 6B), retrolateral teeth occupying basal third of chelicerae, arranged in a row (Fig. 5A) or randomly (Fig. 5B), except I. harti, with retrolateral teeth absent (Fig. 5C), and rastellum consisting of a distinct process with large spines (Fig. 6C). Legs with three tarsal claws, paired claws with teeth and third claw smooth (Fig. 6D). Adhesive scopula absent, pseudoscopula present in males (Fig. 6D). Sternum with two anterior pairs of small marginal sigillae. Abdomen oval, without
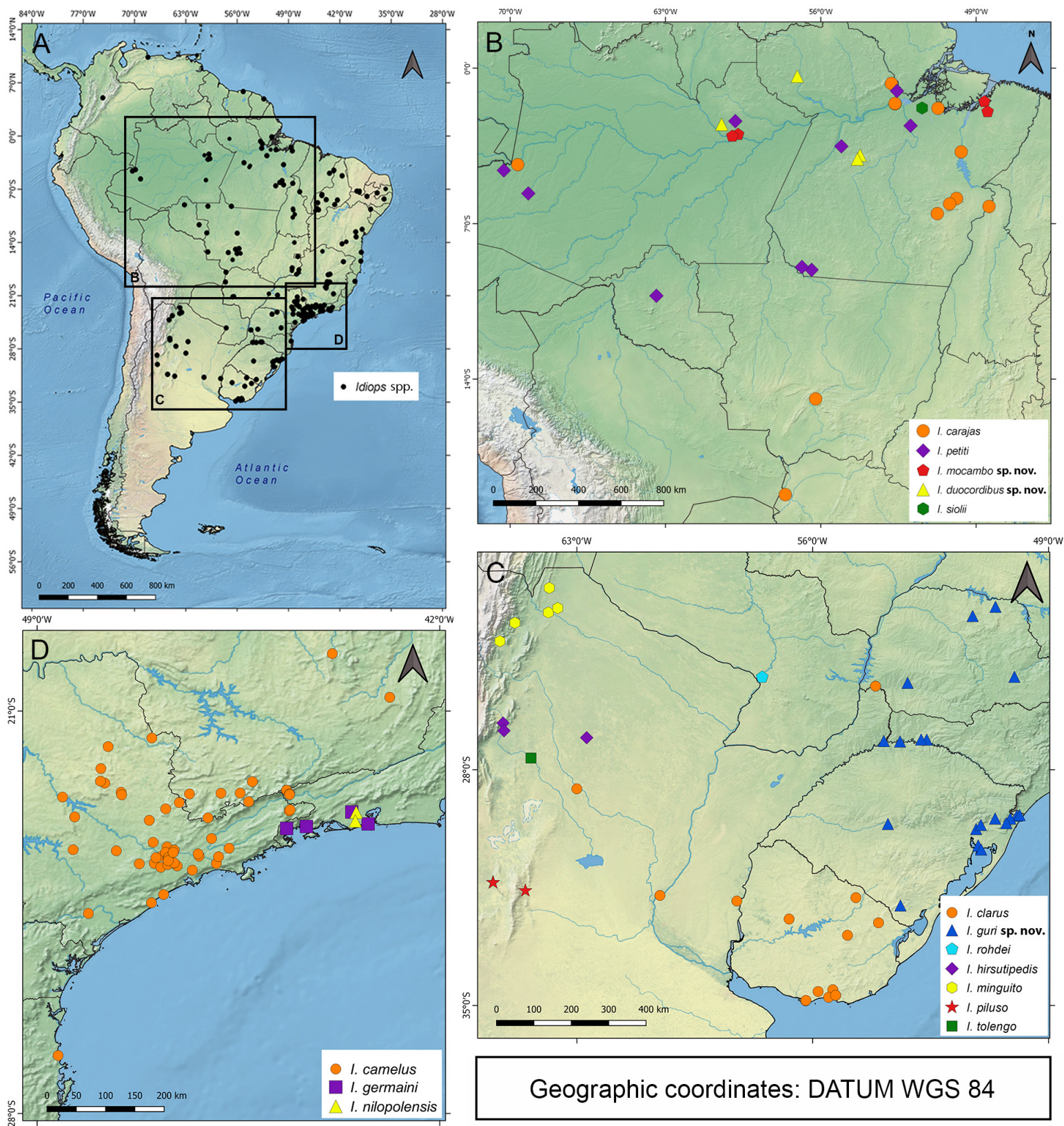

Fig. 3. Distribution of species of Idiops Perty, 1833 in the Neotropical region (part). 
ornaments or sclerotic spots (Fig. 2A-F). Four spinnerets; posterior lateral spinnerets trisegmented and with a domed apical segment, posterior median spinnerets with one short segment. Males with enlarged palpal tibia, with retrolateral depression bordered by robust spines (Fig. 6E-F). Copulatory bulb presents an embolus with median curvature, subapical torsion, apical expansion, usually in flattened shape (embolar lamella) (Fig. 7E-F) or pointed shape (distal embolar tooth) (Fig. 19E) (with the exception of I. petiti; Fig. 26E-F). Leg I with a double tibial apophysis, with apical branch wider than basal branch (Fig. 6G) (with the exception of I. germaini; Fig. 18H), with putative slit organ between the two branches (Fig. 6HI). Metatarsus I smooth (with the exception of I. duocordibus sp. nov., I. petiti and I. pirassununguensis, which have a subapical prolateral projection (Figs 17I, 26I, 27I)). Idiops females have cuspules on labium and maxillae. In I. duocordibus sp. nov., I. opifex and I. rastratus, cuspules occupy entire ventral region of maxillae (Figs 17K, 29K). Tibia III presents dorsal depression in I. fuscus, I. camelus, I. pirassununguensis and $I$. rohdei. Spermathecae with sclerotized basal area, divided into two outward-sloping ducts, each one connected to rounded terminal receptacles (Fig. 7L) (with the exception of I. duocordibus sp. nov., I. petiti and I. siolii; Figs 17L, 26L, 34C) that have receptacles with lobular expansions.

\section{Natural history}

Spiders of the genus Idiops live in tubular silk-lined burrows, which are closed by a hinged lid (operculum), which functions as a trapdoor (Fig. 1A-F, H-L). The burrows, which can be found on ravines (Fig. 1G)

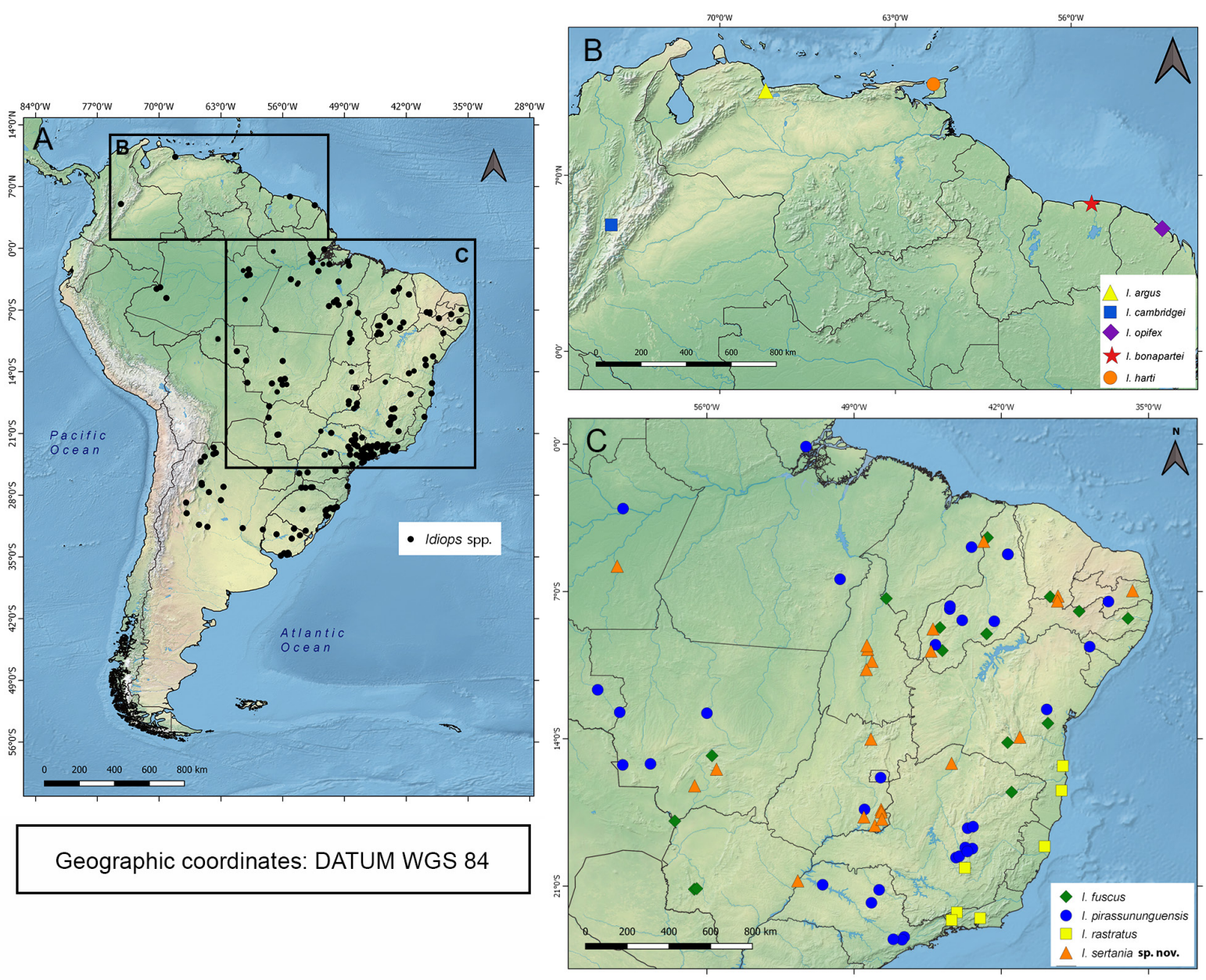

Fig. 4. Distribution of species of Idiops Perty, 1833 in the Neotropical region (part). 
and on the ground (Fig. 1L), are dug with the help of the rastellum and first legs (Coyle et al. 1992). The opercula are usually camouflaged with mosses (Fig. 1A-D), grains of sand and clay (Fig. 6E-F), and small leaves and pieces of dry grass (Fig. 6H-K) (Coyle et al. 1992; Dippenaar-Schoeman 2002). These burrows provide a suitable microclimate that protects the spiders from predators, parasites and microbial infections, and behave as effective expansions for ambushing prey (Dippenaar-Schoeman 2002; Pérez-Miles \& Perafán 2017). Idiops spiders live in a variety of habitats, ranging from dry environments with scattered vegetation and harder soils to more humid environments with dense vegetation and softer soils (Dippenaar-Schoeman 2002; Gupta et al. 2013; RFF pers. obs.). Some species can occasionally be found in termite mounds or in soil deposited near the base of trees (Gupta et al. 2015) and inside tree bark and rotten trunks (Pickard-Cambridge 1889: fig. 5; pers. obs.). Finally, some species can be found in degraded environments, often close to human dwelling and agricultural environments (Mirza \& Sanap 2012; Gupta et al. 2013). In Brazil, specimens of Idiops were found in urban vegetation fragments, located even in large cities, such as São Paulo (I. camelus), Rio de Janeiro (I. germaini) and Brasília (I. pirassununguensis) (RFF pers. obs.).

Females and juveniles spend most of their lives in the burrows, leaving only during events of capturing prey and disposing of undigested parts. Males, after reaching adulthood, leave their burrows and wander in search for females to copulate (Dippenaar-Schoeman 2002; Pérez-Miles \& Perafán 2017). Although less common, it is possible to find males living in individual burrows (Siliwal et al. 2020; RFF pers. obs.). It is common to find several nearby burrows, with spiders often belonging to the same population (DippenaarSchoeman 2002), with records reaching the density of 20 individuals per $\mathrm{m}^{2}$ for I. bombayensis Siliwal, Molur \& Biswas, 2005 (Mirza \& Sanap 2012). At night, the spiders position themselves near the opening, with the trapdoor slightly ajar and wait for the prey that, upon approaching and being noticed, is captured and taken inside (Coyle et al. 1992). If disturbed, the spider closes the operculum firmly, holding the trapdoor with its anterior legs, and as a last resort, goes to the bottom of the burrow (Dippenaar-Schoeman 2002; Pérez-Miles \& Perafán 2017; pers. obs.). In case there is an egg sac inside the burrow, the female positions itself on top of it to better protect its contents. The egg sac is usually oval and can contain up to 250 eggs held together by thick silk lining (as in I. joida Gupta, Das \& Siliwal, 2013) (Gupta et al. 2013). In cases of adverse environmental events, these spiders can abandon their burrows, migrate to apparently more stable environments and build new burrows (Dippenaar-Schoeman 2002; Pérez-Miles \& Perafán 2017).

A

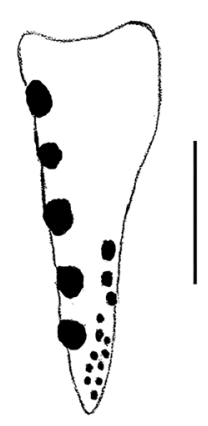

B

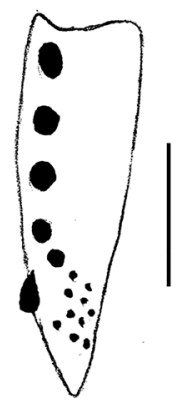

C

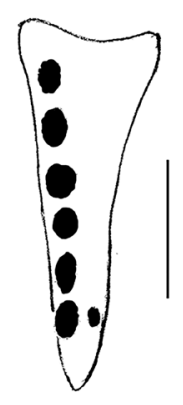

Fig. 5. Chelicera teeth arrangement. A. Smaller retrolateral teeth arranged in rows (I. camelus (MelloLeitão, 1937)). B. Smaller retrolateral teeth arranged randomly (I. germaini Simon, 1892). C. Smaller retrolateral teeth absent (I. harti (Pocock, 1893)). Scale bars $=1 \mathrm{~mm}$. 
Idiops fuscus Perty, 1833

Figs 2A-B, 4C, 6A, 7-8

Idiops fusca Perty, 1833: 197, pl. 39 fig. 5 (ふ).

Sphasus idiops - Walckenaer 1837: 379 (new name for Idiops aculeatus - lapsus). — Petrunkevitch 1911: 780.

Idiops aculeatus - Walckenaer 1837: 379 (lapsus). - Petrunkevitch 1911: 775.

Idiops fuscus - O. Pickard-Cambridge 1870: 103. — Simon 1892: 92, fig. 91.
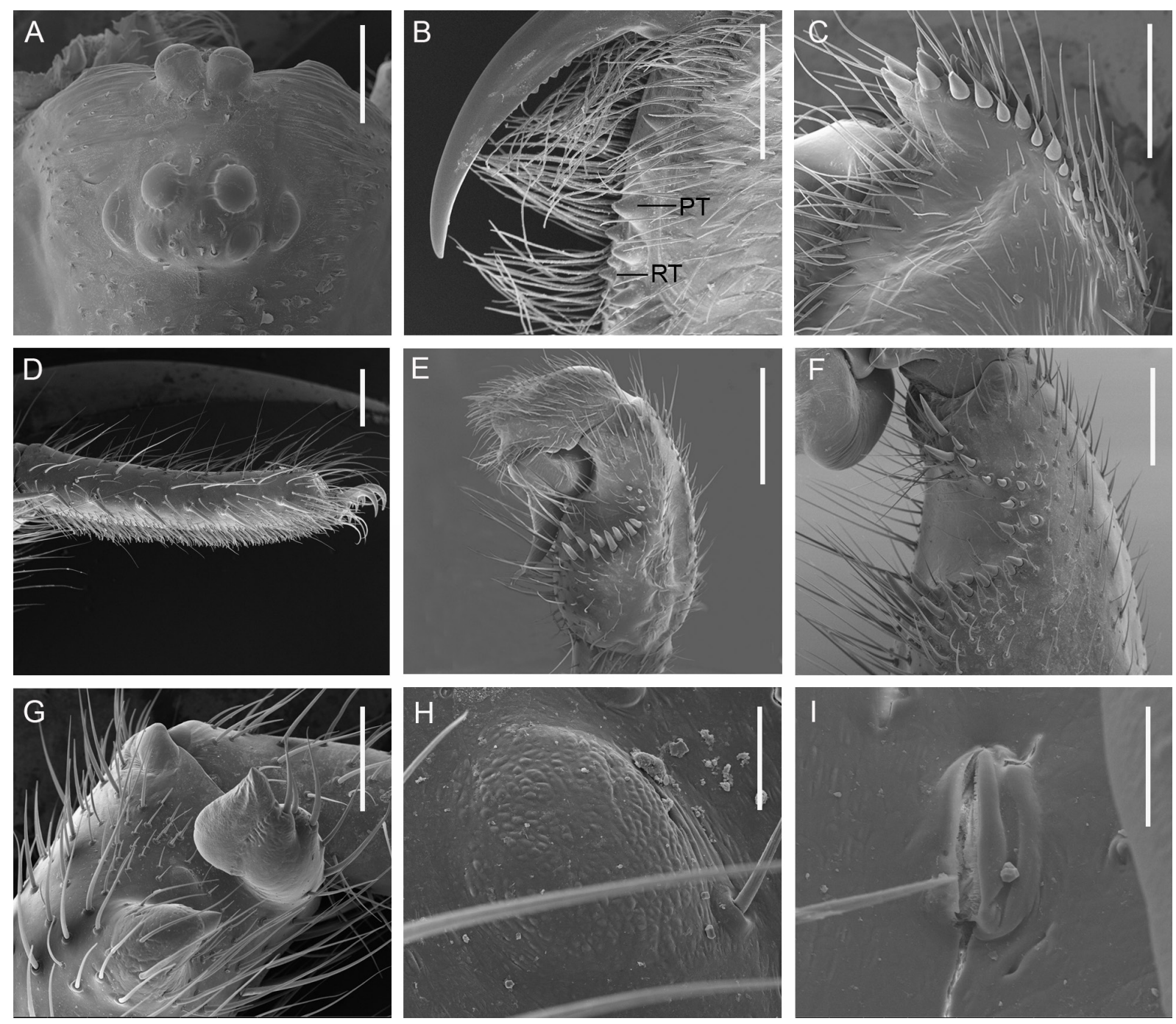

Fig. 6. Diagnostic characters of Idiops Perty, 1833 spp. A. Ocular disposition, with emphasis on the anterior lateral eyes apart from the others (I. fuscus Perty, 1833, CHNUFPI 0036). B. Rows of chelicera teeth (I. clarus (Mello-Leitão, 1946), FCE 0340). C. Rastellum (I. carajas Fonseca-Ferreira, Zampaulo \& Guadanucci, 2017, MPEG 00109). D. Tarsus showing the pseudoscopula and tarsal claws (I. camelus (Mello-Leitão, 1937), MZSP 28832). E. Palpal bulb (I. carajas). F. Retrolateral depression of palpal bulb (I. rastratus (O. Pickard-Cambridge, 1889), MZSP 24215). G. Tibial apophysis (I. pirassununguensis Fukami \& Lucas, 2005, MZSP 15651). H-I. Putative slit organ between the branches of the tibial apophysis (I. camelus). Abbreviations: $\mathrm{PT}=$ promarginal teeth; $\mathrm{RT}=$ retromarginal teeth. Scale bars: A-D, $\mathrm{F}-\mathrm{G}=0.5 \mathrm{~mm} ; \mathrm{E}=1 \mathrm{~mm} ; \mathrm{H}-\mathrm{I}=0.05 \mathrm{~mm}$. 


\section{Diagnosis}

Males of I. fuscus (Figs 7A-I) differ from other Neotropical species of the genus, except I. clarus and I. pirassununguensis, by having palpal tibia with a prominent projection at the base of the retrolateral depression (Fig. 7C). Differs from I. clarus and I. pirassununguensis by the strong curvature of the median portion of the embolus in dorsal view (also present in I. nilopolensis). Differs from I. nilopolensis by the distal portion of the embolus having a pronounced embolar lamella with differentiated dorsal and ventral edges (also present in I. rastratus) (Figs 7D-F, 8E-F). The females differ from other Neotropical species by the aspect of the spermathecae, composed of two ducts slightly curved outward, with a soft and nonsclerotized basal portion and a median and apical portion strongly sclerotized and by the receptacula with a much larger diameter than the duct width (Fig. 7L).

\section{Type material}

\section{Holotype}

BRAZIL - Piauí • MNHN. Should be in the MNHN, lost.

Neotype (here designated)

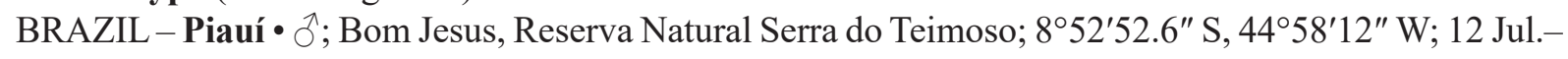
2 Aug. 2000; F. Curcioi and M. Pixo leg.; IBSP 11887.

Remark: Despite an intensive search, the type specimen of Idiops fuscus was not found in the MNHN, being considered lost. In accordance with the criteria of the ICZN Code (ICZN 1999), a neotype is designated here, because the type is lost and the original description is inadequate to stabilize the species. This neotype was based on a specimen collected near the type locality, in the state of Piauí, Brazil.

\section{Other material examined}

BRAZIL - Maranhão • 1 ' ; Carolina, Campus Hotel Rilton; 7¹9'58" S, 47²8'8" W; 10 Dec. 2005; UFMT. - Piauí • 1 q; São Raimundo Nonato; 90'54" S, 4241'56" W; 6 Dec. 1994; R. Bertani and

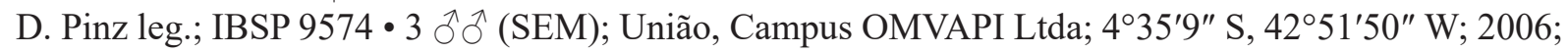

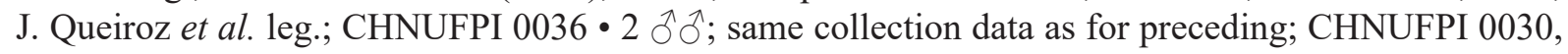

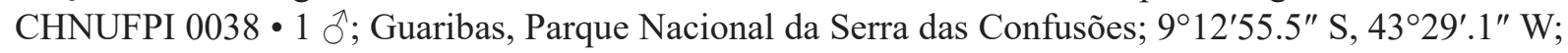
14 Dec. 2010; F.S. Silva leg.; CHNUFPI 4033 • 1 \} \text { ; Guaribas, Parque Nacional da Serra das Confusões; } 9¹3'12.3" S, 43²9'26.7" W; 9-15 Dec. 2010; L.S. Carvalho leg.; CHNUFPI 4034 • 1 ơ; Guaribas, Parque Nacional da Serra das Confusões; 9¹3'32.4" S, 43²7'46.9" W; 10 Dec. 2010; F.S. Silva leg.;

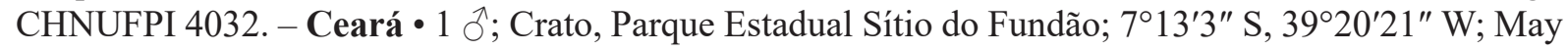

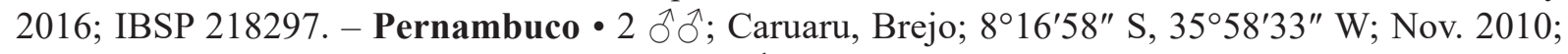

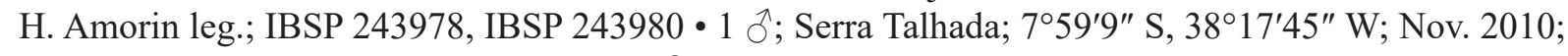
H. Amorin leg.; IBSP 243979. - Bahia • 1 क ; Santa Inês, BR420; 1315'48.7" S, 3947'2.8" W; 18 Jun. 2018; P.H. Martins and E.A. Araújo leg.; UFMG 24028 • 1 क ; Brumado, Magnesita; $14^{\circ} 10^{\prime} 51.5^{\prime \prime}$ S, 4142'0.7" W; 24 Feb. 2017; P. Martins leg.; UFMG 24029. - Mato Grosso • 1 ¡ं; Chapada dos

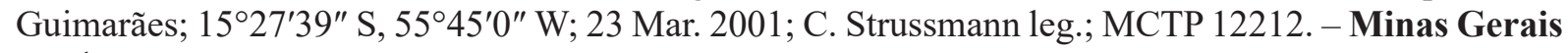

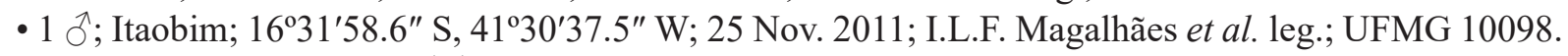

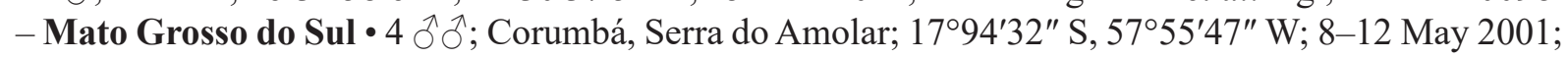
C. Strussmann leg.; MCTP 12193 • 1 q; Bonito; $21^{\circ} 7^{\prime} 15^{\prime \prime}$ S, 56²8'55" W; Oct. 2002; C.A. Rheims leg.;

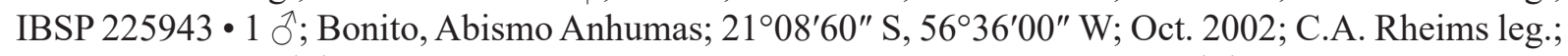
IBSP $243976 \bullet 2 \hat{\jmath}$; same collection data as for preceding; IBSP $243974 \cdot 3 \hat{\jmath}$; same collection data as for preceding; IBSP 243975.

\section{Description}

Male (neotype IBSP 11887)

Habitus. See Fig. 7A. 

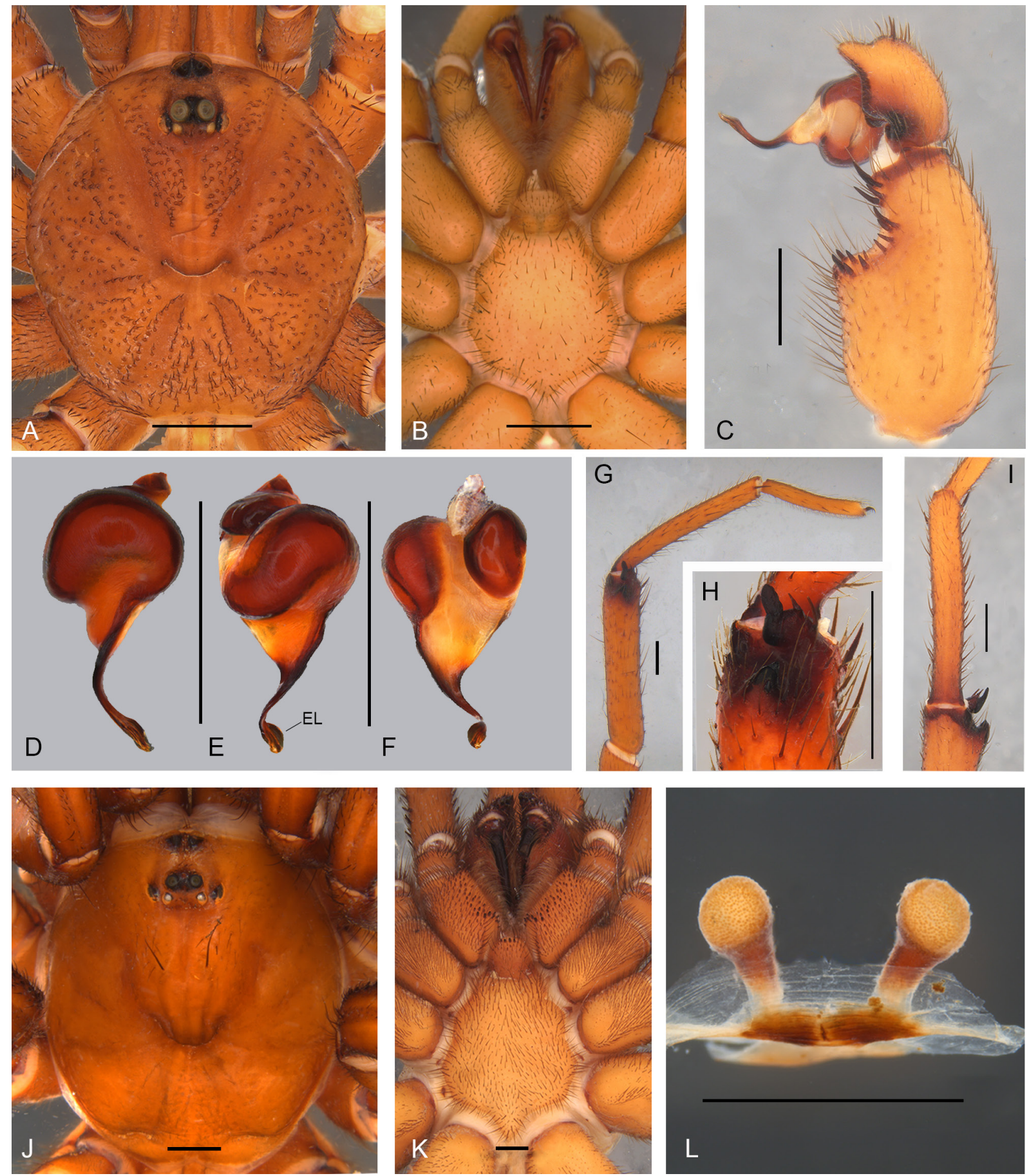

Fig. 7. Idiops fuscus Perty, 1833. A-I. $\widehat{\jmath}$, neotype (IBSP 11887). A. Prosoma (dorsal view). B. Prosoma (ventral view). C. Part of palp (retrolateral view). D-F. Palpal bulb. D. Dorsal view. E. Retrolateral view. F. Prolateral view. G. Tibia, metatarsus and tarsus I (prolateral view). H. Tibial apophysis (prolateral view). I. Tibial apophysis and metatarsus I (dorsal view). J-L. o (IBSP 9574). J. Prosoma (dorsal view). K. Prosoma (ventral view). L. Genitalia (dorsal view). Abbreviation: $E L=$ embolar lamella. Scale bars $=$ $1 \mathrm{~mm}$. 
Measurements. TBL 11.2, CL 5.3, CW 5, LL 0.7, LW 1, SL 3, SW 2.7.

COLOR. Brown carapace and legs, light brown sternum and coxae (Fig. 7A-B), grayish brown abdomen.

Prosoma. Carapace and ocular arrangement as shown in Fig. 7A. Eye distance AME-ALE 0.6. Eye diameters: AME 0.3, ALE 0.3, PME 0.2, PLE 0.3. Thoracic fovea procurved (Fig. 7A). Labium and sternum without cuspules (Fig. 7B). Basal segment of chelicerae with a prolateral row of 10 large teeth and a retrolateral row of 10 small teeth, grouped in basal half, rastellum with 10-12 spines, distal ones longer (Fig. 8A-B).

LEGS. Tibia, metatarsus and tarsus I as shown in Fig. 7G. Leg I with double tibial apophysis composed of a short, thickened basal branch with a small tapered spine and an apical branch composed of a base twice the size of the basal branch, a thickened apical spine followed by two smaller spines (Figs 7H-I, 8C). Pseudoscopula: tarsus I with apical half with weak coverage (Fig. 7G); tarsus II, III and IV fully covered.

PALP. Tibia with conspicuous retrolateral depression and spines distributed along margin (Fig. 7C); embolus with torsion in apical portion, followed by a pronounced lamella, with upper and lower edges with different sizes (Figs 7D-F, 8E-F).

PalP and leg measurements. Palp $=8.8(3,1.4,3,1.4), \mathrm{I}=22.1(6.6,3,4.7,5,2.8), \mathrm{II}=18.9(5.7,2.5$, $3.9,4.4,2.4), \mathrm{III}=16.6(4.5,2.3,2.8,4.6,2.4), \mathrm{IV}=22.7(5.9,2.7,5.2,6,2.9)$.

Spination. Palp: Ti r20, Ta d0-0-8. Leg I: Pa v0-0-4, Mt v0-12, Ta p0-1-1, r1-1-1. Leg II: Ti v0-1-1-2, Mt v1-2-1-3, Ta p1-1-2, r1-1-3. Leg III: Fe d1-2-2, Pa p3-2-4, r0 -0-4, Ti d0-1-0, v1-2-2, p1-1-4, r1-1-2, Mt v2-2-1-3, p1-1-1, r3-1-0, Ta p1-2-3, r0-1-1. Leg IV: Fe d1-1-2, Ti v1-1-3, Mt v1-1-1-3, Ta p1-1-4, r0-0-2.
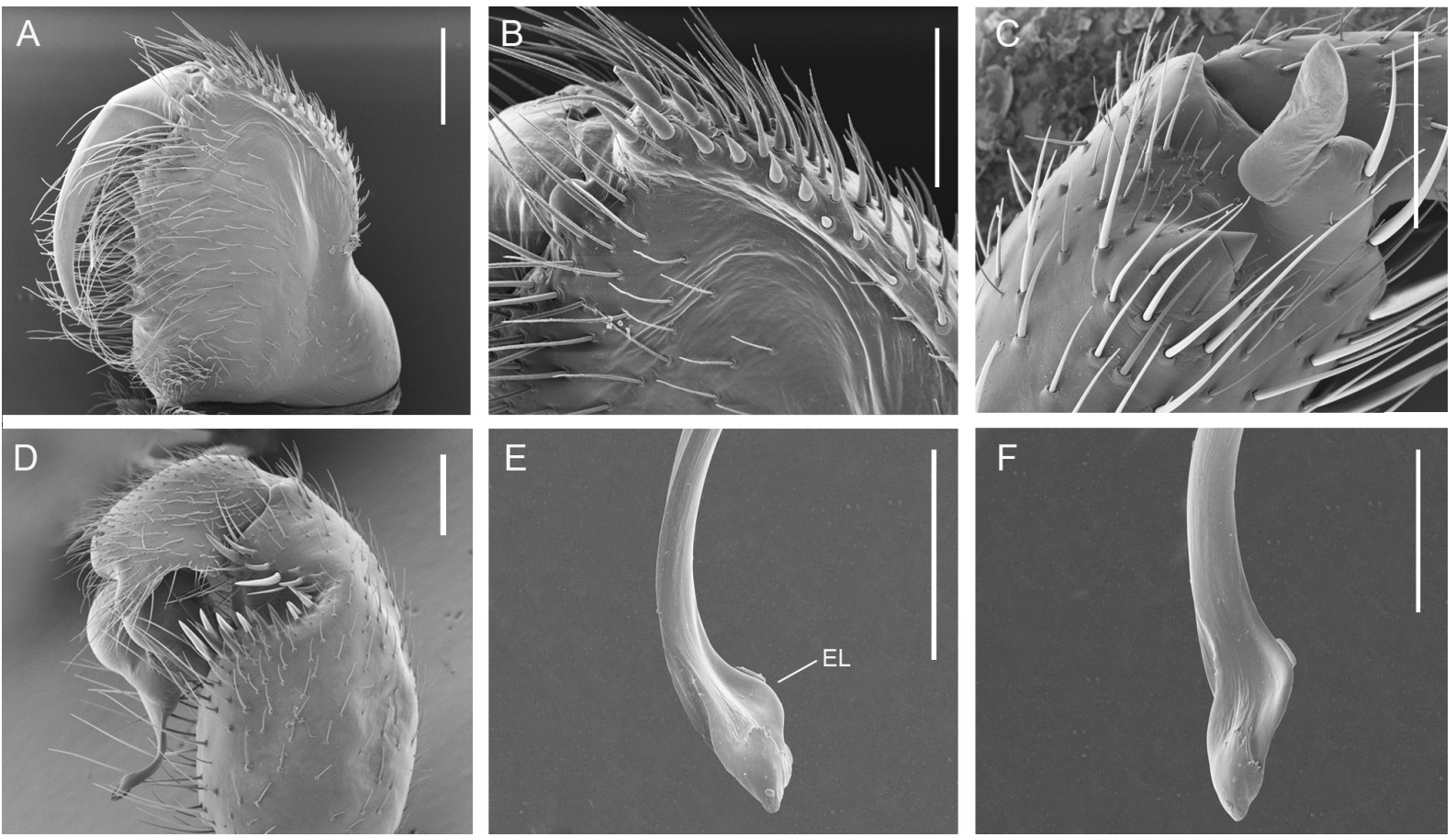

Fig. 8. Scanning electron micrographs of Idiops fuscus Perty, 1833, đ̊ (CHNUFPI 0036). A. Chelicera (retrolateral view). B. Rastellum (retrolateral view). C. Tibial apophysis (dorsal view). D. Part of the palp, showing retrolateral depression. E-F. Detail of the distal portion of the embolus. E. Retrolateral view. F. Prolateral view. Abbreviation: $\mathrm{EL}=$ embolar lamella. Scale bars: $\mathrm{A}, \mathrm{C}-\mathrm{D}=0.5 \mathrm{~mm} ; \mathrm{B}=0.3 \mathrm{~mm}$; $\mathrm{E}-\mathrm{F}=0.2 \mathrm{~mm}$. 
Female (IBSP 9574)

Habitus. See Fig. 7J.

MeAsurements. TBL 18.9, CL 8.2, CW 6.9, LL 1.2, LW 1.5, SL 4.7, SW 4.

CoLOR. Brown carapace and legs, light brown sternum and coxae (Fig. 7J-K), grayish brown abdomen.

Prosoma. Carapace and ocular arrangement as shown in Fig. 7J. Eye tubercle: 1.75 long, 1.83 wide. Distance AME-ALE 1. Eye diameters: AME 0.4, ALE 0.4, PME 0.3, PLE 0.5. Thoracic fovea procurved (Fig. 7J). Labium with 7 cuspules (Fig. 7K). Maxilla with 56 cuspules, distributed over anterior ventral half (Fig. 7K). Basal segment of chelicerae with a prolateral row of 10 large teeth and a retrolateral row of 10 small teeth, grouped in basal half. Robust rastellum, presenting 18-20 short and thick spines on a tubercle.

Palp and leg measurements. Palp $=12.3(4.2,2.7,2.8,2.6), \mathrm{I}=13.6(4.6,2.9,2.9,2.3,0.9), \mathrm{II}=12.95$ $(4.3,2.9,2.5,2.2,1), \mathrm{III}=13.8(3.7,3.1,2.3,2.9,1.85), \mathrm{IV}=19.4(5.4,3.8,4.1,4,2.1)$.

Sination. Palp: Fe p0-0-2, Pa p0-0-1, Ti p3-6-7, r3-5-12, Ta v0-0-7, p7-7-7, r11-9-7. Leg I: Ti p3-4-8, r3-6-12, Mt p9-6-5, r8-7-5, Ta v0-0-5, p4-3-2, r4-2-2. Leg II: Ti p3-3-5, r1-1-1, Mt p9-7-7, r3-3-2, Ta v0-0-3, p4-5-2, r3-1-0. Leg III: Pa p3-5-10, r0-0-2, Ti v0-0-2, p1-3-3, r0-2-6, Mt v1r-1r-2, p7-1-1, r7-2-1, Ta v0-3-8. Leg IV: Pa p17-0-0, Ti v1-1-1, Mt v1-1-1-3, Ta v0-3-8.

Spermathecae. Duct with apical half thicker than its basal half, oval receptacula (Fig. 7L).

\section{Distribution}

Brazil. Widely distributed, occurring in areas with phytophysiognomies in the Caatinga and Cerrado. Records for Central-West region (Mato Grosso, Mato Grosso do Sul), Northeast region (Bahia, Ceará, Maranhão, Paraíba, Pernambuco and Piauí) and the northern part of Minas Gerais (Fig. 4C).

Idiops argus Simon, 1889

Figs 4B, 9

Idiops argus Simon, 1889: 180, pl. 2 fig. 1.

Idiops fulvipes Simon, 1889: 181. Syn. nov.

Idiops argus - Dupérré \& Tapia 2021: 272, fig. 14a-d.

Idiops fulvipes - Dupérré \& Tapia 2021: 273, fig. 15a-d.

\section{Diagnosis}

Females of Idiops argus differ from the other Neotropical species by having the spermathecae with a unique morphology within the genus, with a long and spiral duct followed by an oval receptacle (Fig. 9).

\section{Type material}

Lectotype (here designated)

VENEZUELA - Carabobo • O; Puerto Cabello, San Esteban; 1889; E. Simon leg.; MNHN 4169.

\section{Paralectotypes}

VENEZUELA - Carabobo • 1 , , 2 juvs; same collection data as for lectotype; MNHN 4169a. 


\section{Holotype of I. fulvipes Simon, 1889}

VENEZUELA - Miranda • juv.; La Silla de Caracas, Parque Nacional El Ávila; $10^{\circ} 32^{\prime} 0^{\prime \prime}$ N, $66^{\circ} 52^{\prime} 0^{\prime \prime}$ W; 1889; MNHN.

Remark: The type specimen of Idiops fulvipes was examined and recognized as a juvenile of I. argus. Although the spermathecae were presented by Dupérré \& Tapia (2021: fig. 15c-d), we detected that it is a pre-adult female of I. fulvipes.

\section{Description}

\section{Male}

Unknown.

\section{Female (MNHN 4169)}

Measurements. TBL 13.7, CL 6.7, CW 5.6, LL 0.9, LW 1.2, SL 3.9, SW 3.5.

CoLOR. Carapace, sternum and legs brownish. Abdomen ventrally brownish and dorsally gray.

Prosoma. Eye tubercle: 1.9 long; 1.7 wide. AME-ALE distance 1.2. Eye diameters: AME 0.3, ALE 0.5, PME 0.3, PLE 0.4. Thoracic fovea procurved. Labium with six cuspules. Maxilla with 54 cuspules, distributed over anterior ventral half. Basal segment of chelicerae with a prolateral row of 8 large teeth and 6 retrolateral small teeth, grouped in basal half.

PalP And leg measurements. Palp $=11.4(3.9,2.4,2.4,2.4), \mathrm{I}=12.48(4.04,2.80,2.64,2.04,0.96)$, $\mathrm{II}=11.1(3.68,2.52,2.16,1.84,0.92), \mathrm{III}=11.2(3.2,2.6,1.8,2,1.6), \mathrm{IV}=15.9(4.3,3.1,3.5,3.2,1.8)$.

SPInATion. Palp: Fe p0-0-1, Pa p0-0-1, r0-0-1, Ti p8-7-13, r7-7-12, Ta v0-0-3, p13-12-9, r13-8-9. Leg I: Ti p6-8-10, r6-7-15, Mt p12-10-7, r11-9-8, Ta v0-1-6, p4-4-2, r5-4-2. Leg II: Ti p3-5-8, r1-1-1, Mt p1311-6, r1-2-2, Ta v0-2-4, p5-4-3, r4-2-1. Leg III: Pa p6-7-12, r0-0-3, Ti v2-2-2, p2-3-6, r1-2-4, Mt v2-2-2, p5-3-2, r2-2-1, Ta v0-4-11. Leg IV: Pa p29-3-0, Ti v1-1-2, Ta v4-5-10.

SpermatheCAe. Long spiral ducts and bean-shaped receptacula (Fig 9).

\section{Distribution}

Venezuela. Carobobo and Miranda (Fig. 4B).

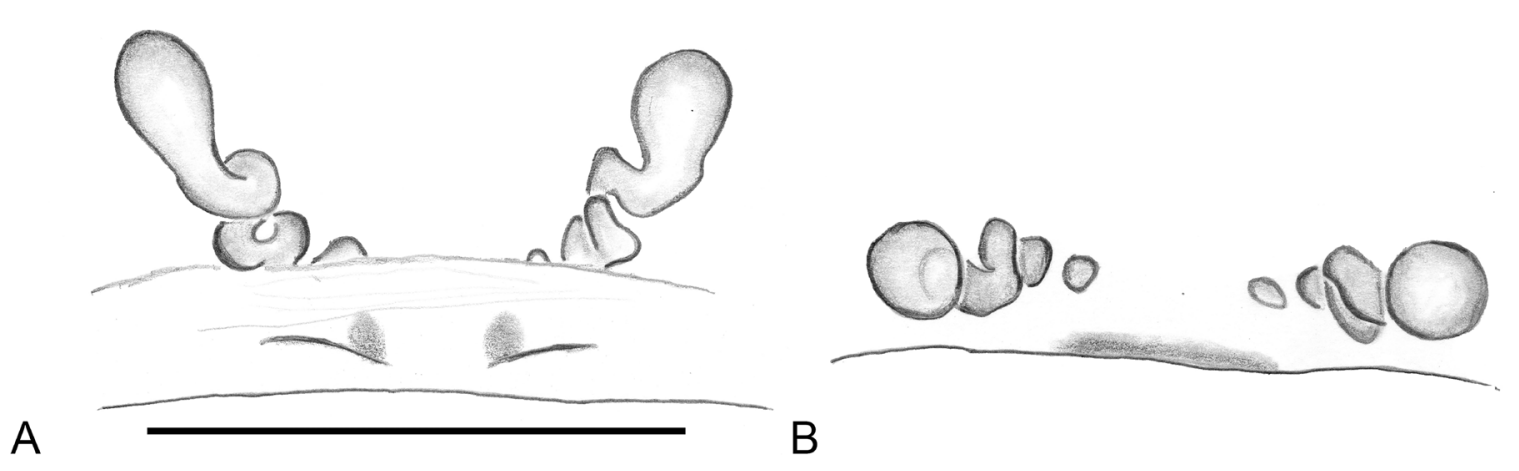

Fig. 9. Idiops argus Simon, 1889, +, genitalia, lectotype (MNHN 4169). A. Dorsal view. B. Frontal view. Scale bars $=1 \mathrm{~mm}$. 
Idiops cambridgei Ausserer, 1875

Figs 4B, 10

Idiops cambridgei Ausserer, 1875: 145.

Idiops cambridgei - Raven 1985: 158.

Pseudidiops cambridgei - Pocock 1895: 223.

\section{Diagnosis}

The female of Idiops cambridgei differs from that of the other Neotropical species by the spermathecae having ducts with strong outward curvature in the transition between duct and receptacle, and small receptacula, with diameter slightly larger than the width of the duct (Fig. 10).

\section{Type material}

\section{Holotype}

COLOMBIA • +; Bogotá; 1889; BMNH 1890.7.1.321.

\section{Description}

Male

Unknown.

Female (holotype BMNH 1890.7.1.321)

MeASurements. TBL 12.9, CL 7, CW 5.4, LL 0.9, LW 1.2, SL 3, SW 3.

CoLor. Carapace and legs brown. Sternum, coxae and abdomen brownish.

Prosoma. Eye tubercle: 1.4 long; 1.3 wide. AME-ALE distance 0.5. Eye diameters: AME 0.3, ALE 0.3, PME 0.2, PLE 0.3. Thoracic fovea procurved. Labium with 16 cuspules. Maxilla with 114 cuspules, distributed throughout ventral area. Basal segment of chelicerae with a prolateral row of 6 large teeth and 3 retrolateral small teeth, grouped in basal half.

PalP And Leg Measurements. Palp $=8.3(2.9,1.8,1.8,1.8), \mathrm{I}=9.9(3.4,2.4,1.9,1.3,0.9), \mathrm{II}=8.7(2.9$, $2,1.7,1.3,0.8), \mathrm{III}=8.4(2.4,1.9,1.5,1.6,1), \mathrm{IV}=11.2(3.3,2.3,2.4,2.1,1.1)$.

Spination. Palp: Fe p0-0-3ap, r0-0-3ap, Pa p0-0-1, Ti p5-5-7, r2-4-7, Ta p7-6-6, r8-9-8. Leg I: Fe r0-0-3, Ti p5-5-5, r4-4-8, Mt p7-6-7, r3-3-2, Ta p3-2-1, r2-1-1. Leg II: Ti p2-4-3, r3-3-1, Mt p10-6-4, r4-3-1, Ta

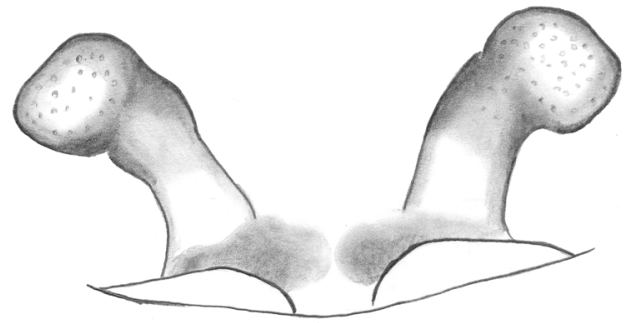

A

Fig. 10. Idiops cambridgei Ausserer, 1875, $q$, genitalia, holotype (BMNH 1890.7.1.321). A. Dorsal view. B. Frontal view. Scale bars $=1 \mathrm{~mm}$.

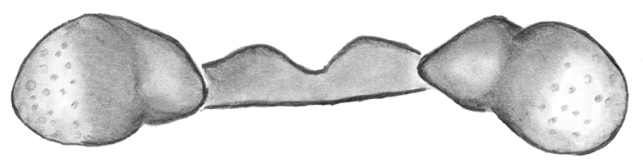

B 
p3-2-2, r2-0-0. Leg III: Pa p5-9-25, r0-1-1, Ti p5-13-19, r4-4-6, Mt v0-1-2, p8-5-5, r5-0-1, Ta v0-0-4. Leg IV: Pa p31-31-23, Ti v0-0-2, Mt v1-2-3ap, Ta v0-3-3.

Spermathecae. Spherical receptacula. Duct with basal translucent area and median area sclerotized (Fig. 10).

\section{Distribution}

Colombia. Known only from the type locality (Fig. 4B).

Idiops camelus (Mello-Leitão, 1937)

Figs 2C-D, 5A, 6D, H-I, 11-12

Pseudidiops camelus Mello-Leitão, 1937: 1, figs 1-2.

Idiops montealegrensis Soares, 1944: 156, figs 2-4. Synonymized by Fukami \& Lucas 2005: 6, figs 6-7.

Idiops camelus - Raven 1985: 158. — Fukami \& Lucas 2005: 6, figs 6-7.

Idiops montealegrensis - Bücherl 1957: 383, figs 2-2a.

\section{Diagnosis}

Males of Idiops camelus (Fig. 11A-I, 12) differ from other Neotropical species by having the apical half of the embolus tapering (Fig. 11D-F), with the presence of a distal embolar tooth (also present in I. clarus and I. germaini) close to the opening of the sperm duct (Fig. 12E-F). Differs from I. clarus and I. germaini by having the apical portion of the embolus more expanded (Fig. 12E-F). Females (Fig. 11 J-L) have spermathecae with spherical receptacles slightly wider than the ducts, with a subtle constriction between duct and receptacle (Fig. 11L).

\section{Type material}

Holotype of Pseudidiops camelus Mello-Leitão, 1937

BRAZIL - São Paulo・ • ; Corumbataí; IBSP 3429.

Holotype of Idiops montealegrensis Soares, 1944

BRAZIL - São Paulo • ${ }^{\lambda}$; Amparo, Monte Alegre, Fazenda Santa Maria; Nov. 1942; F. Lane leg.; MZSP 326.

\section{Other material examined}

BRAZIL - Minas Gerais • 1 q; Lima Duarte, Parque Estadual de Ibitipoca; $21^{\circ} 43^{\prime} 0^{\prime \prime}$ S, $43^{\circ} 54^{\prime} 0^{\prime \prime}$ W; 21 Oct. 1995; R. Baptista leg.; MNRJ • 1 q; Monte Verde, trail south of Monte Verde; 22 $51^{\prime} 27^{\prime \prime}$ S,

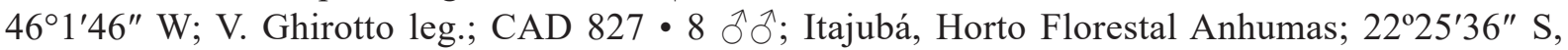

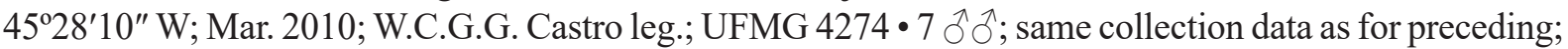
UFMG $4270 \bullet 7$ ô; same collection data as for preceding; UFMG $4272 \cdot 7 \hat{\jmath}$; ; same collection data as for preceding; UFMG $4275 \bullet 8 \hat{\jmath} \hat{\jmath}$; same collection data as for preceding; UFMG $4273 \bullet 8 \hat{\jmath}$; same collection data as for preceding; UFMG $4271 \cdot 2 \delta^{\top} \delta^{\top}$; Cristina, Parque Ecológico Municipal; $22^{\circ} 12^{\prime} \mathrm{S}$, $45^{\circ} 11^{\prime}$ W; Sep. 2009; C.G.G. Castro leg.; UFMG 4232 • 1 ``; Nova Lima, RPPN Mata Samuel de Paula; $20^{\circ} 00^{\prime} \mathrm{S}$, $43^{\circ} 52^{\prime} \mathrm{W}$; 18 Oct. 2006; J.P.P.P. Barbosa et al. leg.; UFMG 2530 • 1 क; Bueno Brandão, $1.5 \mathrm{~km}$

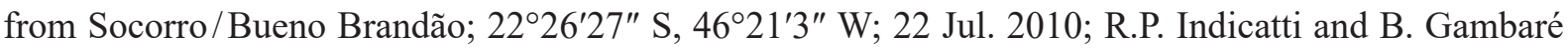

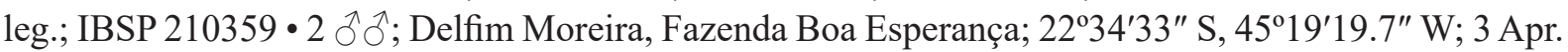
2014; A. Nogueira et al. leg.; IBSP 210175 • 1 q; Viçosa, Campus UFV, Recanto de Cigarras; 2045'37" S, 42 52'4" W; 6 Mar. 2013; E.S. Barreto, A.A. Zaccaro and G.M. Diniz leg.; IBSP 221797. - Rio de Janeiro

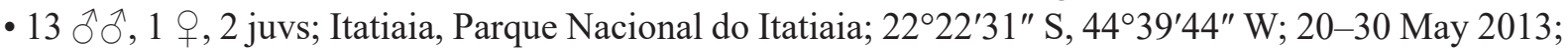


R.P. Indicatti and F.U. Yamamoto leg.; IBSP 225155 • 1 \%; Itatiaia, Parque Nacional do Itatiaia;

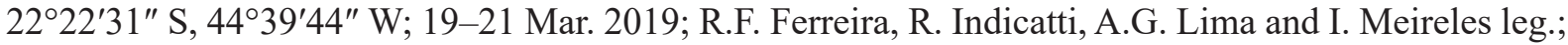
CAD 289 • 1 गे; Itatiaia, Parque Nacional do Itatiaia, Cachoeira Poranga; $22^{\circ} 26^{\prime} 25.7^{\prime \prime} \mathrm{S}, 44^{\circ} 36^{\prime} 40^{\prime \prime} \mathrm{W}$; 17 Apr. 2014; R. Pinto da Rocha, A. Benedeti, D. Chiviri and J. Cabra leg.; IBSP 210439 • 2 ふ̋ ô; Itatiaia,

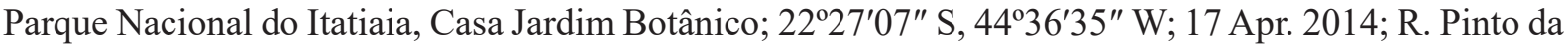
Rocha, A. Benedeti, D. Chiviri and J. Cabra leg.; IBSP 210440. - Sao Paulo • 2 $9+$; São José do Barreiro,

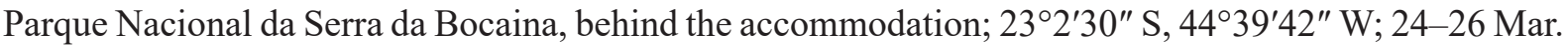
2019; R.F. Ferreira, A. Galleti, I. Meirelles and R. Indicatti leg.; CAD 830 • 1 đ’; São José do Barreiro,

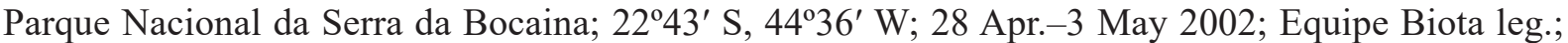

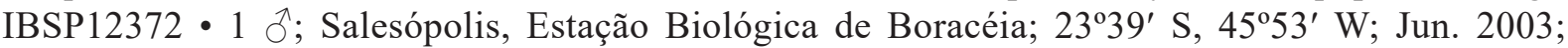
J.P.L. Guadanucci leg.; MZSP 27668• 1 Ō; Salesópolis, Estação Biológica de Boracéia; MZSP 21159 • 2 ổ; Salesópolis, Estação Biológica de Boracéia; 11 May. 2003; J.P.L. Guadanucci leg.; MZSP 22005 - 8 ठో ठే; Salesópolis, Estação Biológica de Boracéia; 7 Mar. 2003; J.P.L. Guadanucci leg.; MZSP 23714 - 1 P; Salesópolis, Estação Biológica de Boracéia; Dec. 1949; I. Travassos leg.; MZSP 11326 • 1 đే; Salesópolis, Estação Biológica de Boracéia; Mar. 2006; M.U. Prado et al. leg.; IBSP 14547 • 1 \%; MogiSalesópolis Road, Km 91; 25 Jun. 2002; R. Martins et al. leg.; IBSP 13111 • 1 đ;; Amparo, Fazenda Santa

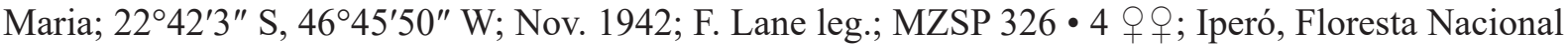
de Ipanema; $23^{\circ} 25^{\prime} 49^{\prime \prime} \mathrm{S}, 47^{\circ} 37^{\prime 2} 22^{\prime \prime} \mathrm{W}$; 10-14 Oct. 2019; J.P.L. Guadanucci, R.P. Indicatti and A. Galleti-

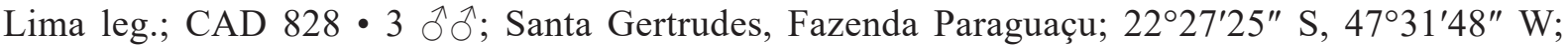
J.H.B. Medeiros leg.; IBSP 243977 • 1 o ; Barra Bonita; 22²9'42" S, 48³3'28" W; 6 Nov. 1979;

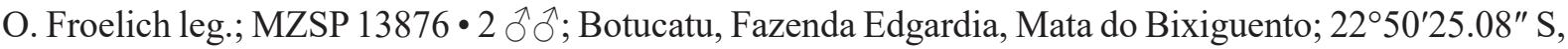

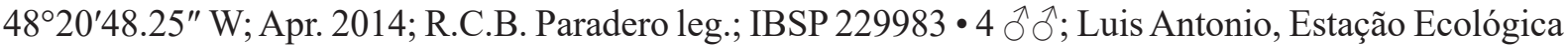

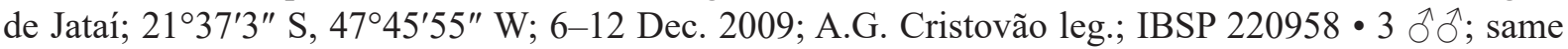
collection data as for preceding; IBSP $220944 \bullet 1$; same collection data as for preceding; IBSP 220949 - 3 సో ठ; Luis Antonio, Estação Ecológica de Jataí; 3-7 Oct. 2009; A.G. Cristovão leg.; IBSP 220948 •

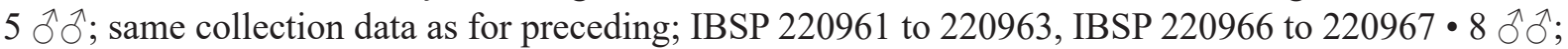
Luis Antonio, Estação Ecológica de Jataí; Jan. 2010; A.G. Cristovão leg.; IBSP 243964, IBSP 243966 to

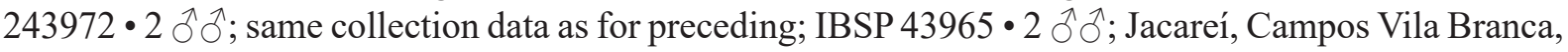

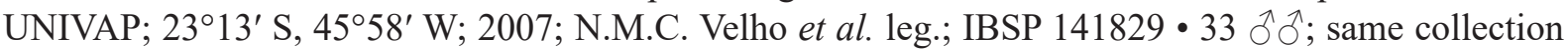
data as for preceding; IBSP 141786, IBSP 141788 to 141791, IBSP 141796, IBSP 141798 to 141800 , IBSP 141804 to 141810 , IBSP 141818 to 141821 , IBSP 141823 to 141828 , IBSP 141831 to 141832 ,

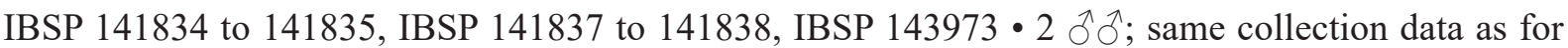

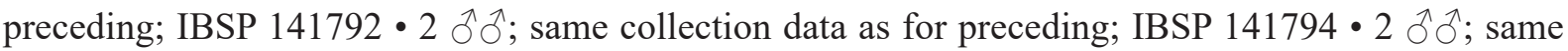

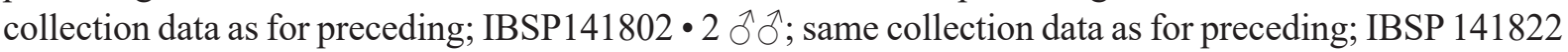

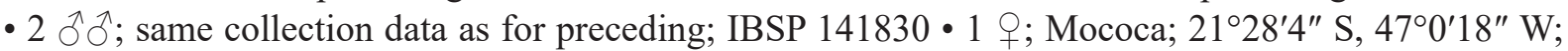
6 Mar. 1991; J.A. Azevedo leg.; IBSP 11834 • 1 o $^{\prime}$; Itapecerica da Serra; $23^{\circ} 43^{\prime} 1^{\prime \prime} \mathrm{S}, 46^{\circ} 50^{\prime} 56^{\prime \prime} \mathrm{W}$; IBSP

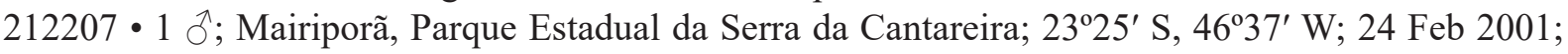

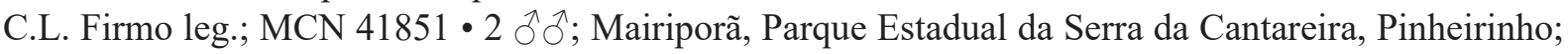

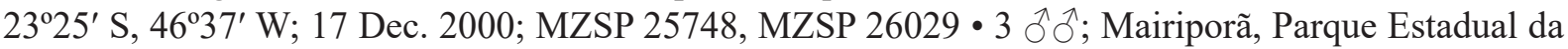
Serra da Cantareira, Pinheirinho; 24 Feb. 2001; MZSP 26030 to $26032 \cdot 2$ o $\sigma^{\wedge}$; Mairiporã, Parque Estadual da Serra da Cantareira, Pinheirinho; 27 Apr. 2001; MZSP 26035 to 26036 • 1 đ̊; Mairiporã, Parque Estadual da Serra da Cantareira, Pinheirinho; 1 Jul. 2001; MZSP 26033 • 1 đ; Mairiporã, Parque Estadual da Serra da Cantareira, Pinheirinho; 27 May 2001; MZSP 26034 • 1 ô; $^{\prime}$ Paraibuna, Sitio Amici; $23^{\circ} 23^{\prime} 9^{\prime \prime}$ S, 4539'43" W; 13 Jul. 2003; M.B. da Silva leg.; MZSP 21820 • 1 o ; Jaú; 22¹7'45" S, 48³3'28" W; 21 Dec. 2011; A.M. Giroti leg.; IBSP 210069 • 1 ơ; Paraibuna, Sitio Amici; 2008; A.M. Giroti leg.; IBSP

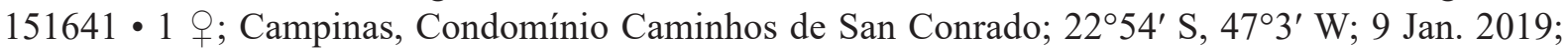
V. Ghirotto leg.; CAD 831 • 1 क; Campinas; 24 Aug. 1974; O. Froelich leg.; MZSP $13880 \bullet 1$ đ̊; Jundiaí, Parque Estadual Serra do Japi; $23^{\circ} 17^{\prime}$ S, 46 59' W; 13 Nov. 2007; J. Sobjac leg.; UFMG 6475 • 1 §’;

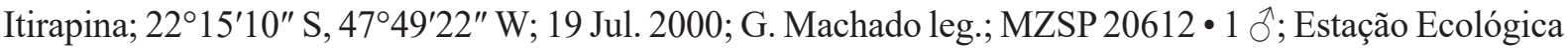

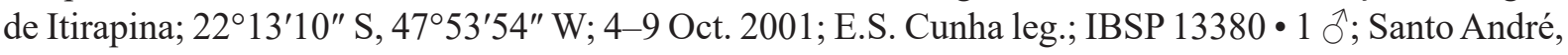




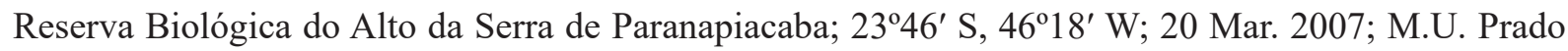
et al. leg.; IBSP 14566 • 1 \%; Santo André, Reserva Biológica do Alto da Serra de Paranapiacaba; 17 Nov. 2006; M.U. Prado et al. leg.; IBSP 14567 • 1 क ; São Carlos; $22^{\circ} 0^{\prime} 0^{\prime \prime}$ S, 4753'27" W; 9 Jun. 1946; F. Salto

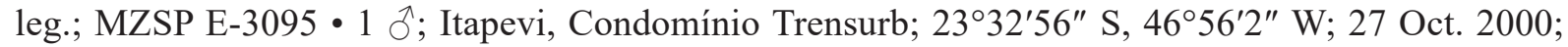
V. Onofrio and D.M.B.B. Batesti leg.; IBSP $123524 \bullet 1$ \%; Itapevi, Condomínio Trensurb; 2000; V. Onofrio and D.M.B.B. Batesti leg.; IBSP 123525 • 1 क; Mogi das Cruzes; $23^{\circ} 31^{\prime} 26,27^{\prime \prime}$ S, 46 $6^{\circ} 11^{\prime} 0,685^{\prime \prime}$ W; 30 Oct.

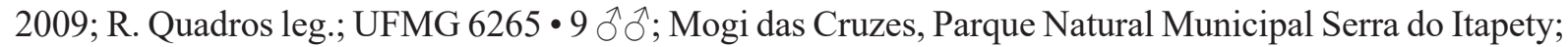
$23^{\circ} 29^{\prime}$ S, $46^{\circ} 12^{\prime}$ W; 13 Nov.-19 Dec. 2003; Equipe Biota leg.; IBSP 13391, IBSP 13393, IBSP 13395 to 13396, IBSP 13398 to 13399,13401 to $13403 \cdot 1$; ; same locality as for preceding; 5 Jun. 2003; IBSP

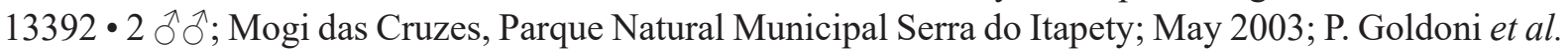
leg.; IBSP 10189 to 10190 • 1 ; ; Itapevi, Parque Natural Municipal Serra do Itapety; Feb.-Mar. 2004; P. Goldoni et al. leg.; IBSP $11781 \cdot 2$ ồ; Itapevi, Parque Natural Municipal Serra do Itapety; 2014-2015;

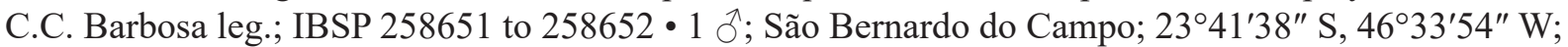
17 Apr. 2009; Pref. Mun. SB Campo leg.; IBSP 151640 • 2 q 9 ; Socorro, Gruta do Anjo; 22 $35^{\prime 2} 27^{\prime \prime}$ S, 46³1'44" W; 19-26 Dec. 2010; R.P. Indicatti and B. Gambaré leg.; IBSP 210350 to 210351 • 1 q; São

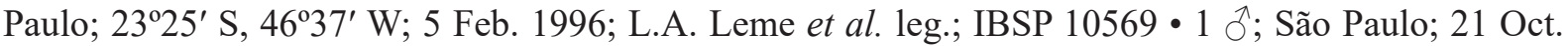
2004; R.M. Nori leg.; IBSP $11040 \bullet 1$ đ; São Paulo; 20 Oct. 2002; D.P.C. Souza leg.; IBSP $9961 \bullet 1$ đ; São Paulo; Oct. 1971; A. Guarnieri leg.; IBSP 2113 • 1 ô; São Paulo; 3 Oct. 1991; N. Cochrane leg.; IBSP 8256 • 1 क; São Paulo; 1937; A. Franci leg.; IBSP 3723 • 1 q; São Paulo, Serra da Cantareira; 27 Aug. 1999; F.L. da Silva leg.; IBSP 9579 • 1 ô; São Paulo, Parque Estadual da Serra da Cantareira;

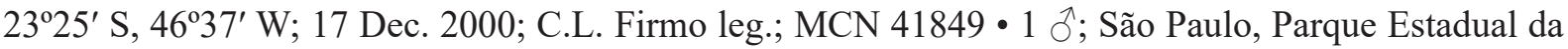
Serra da Cantareira; 27 Apr. 2001; A.D. Brescovit leg.; MCN 41850 • 11 ổ ô; São Paulo, Parque Estadual da Serra da Cantareira; 3 Mar.-5 Jul. 2000; S. Favorito et al. leg.; MZSP 21316 • 2 ô ô; same collection

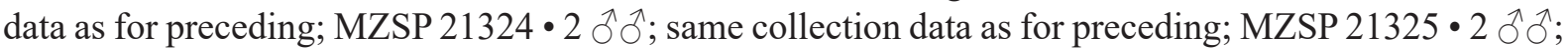
same collection data as for preceding; MZSP 21314 3 त; same collection data as for preceding; MZSP $21322 \cdot 6 \widehat{\partial}$; same collection data as for preceding; MZSP 21323・2 $\widehat{\partial}$; same collection data as for

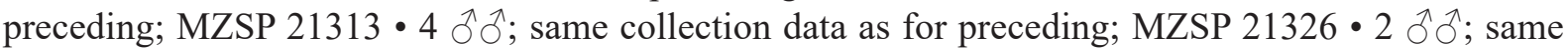
collection data as for preceding; MZSP • 2 ठ $\sigma^{\lambda}$; same collection data as for preceding; MZSP $21309 \bullet$

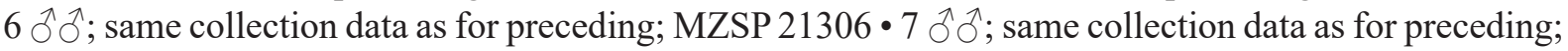

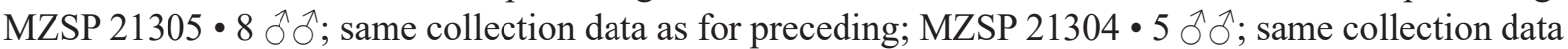
as for preceding; MZSP 21320 • 18 $\hat{\jmath}$; same collection data as for preceding; MZSP 21307, MZSP 21315, MZSP 21329, MZSP 21333, MZSP 21335 to 21336, MZSP 21338 to 21342, MZSP 21345 to 21346, MZSP 21348 to $21352 \cdot 2$ 우; same collection data as for preceding; MZSP 21311, MZSP 21330

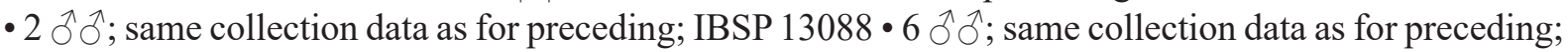
IBSP 13082, IBSP 13084, IBSP 13087, IBSP 13089, IBSP 13094 to 13095, IBSP 13095 • 1 đ ; São Paulo, Parque Estadual da Serra da Cantareira; 29 Apr. 2001; R. Pinto da Rocha leg.; MCN 41848 • 1 q; São Paulo, Parque Estadual da Serra da Cantareira; 18 Aug. 1999; F.L. da Silva leg.; IBSP 7913 • 1 đ̇; São Paulo, Parque Estadual da Serra da Cantareira; 24 Jan. 2001; F.L. da Silva leg.; IBSP 130688 • 1 ô; same locality as for preceding; 27 Apr. 2001; F.L. da Silva leg.; IBSP $130689 \bullet 1$ đ’; São Paulo, Parque Estadual da Serra da Cantareira; 1 Aug. 2001; F.L. da Silva leg.; IBSP $130690 \bullet 1$ đ̧; same locality as for preceding;

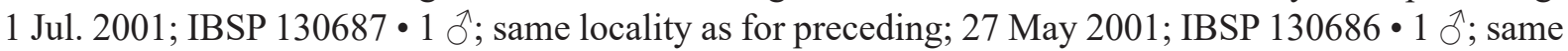
collection data as for preceding; IBSP $130691 \bullet 9 \widehat{\jmath}$; ; São Paulo, Parque Estadual da Serra da Cantareira; 17 Jul. 2000-1 Aug. 2001; C.L. Firmo leg.; MZSP 24046, MZSP 26027, MZSP 26037, MZSP 26039 to 26040, MZSP 26042, MZSP 26044 to 26046 2 ô ${ }^{\text {; }}$; same collection data as for preceding; MZSP 26041,

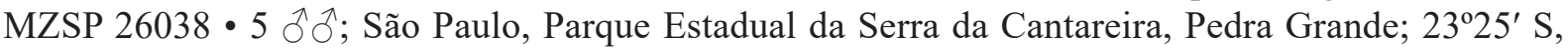
46³7' W; 17 Dec. 2000-1 Jul. 2001; C.L. Firmo leg.; MZSP 26023 to 26026, MZSP 26028 • 1 đ̊; São Paulo, Parque Estadual da Serra da Cantareira, Pedra Grande; 25 Aug.-3 Sep. 2000; C.C. Aires et al. leg.; MZSP 21328 • 1 क ; São Paulo, Parque Estadual do Jaraguá; 2327'34.3" S, 4646'2.8" W; Apr. 2015; R.P. Indicatti and J.P.L. Guadanucci leg.; CAD 598 • 1 क; same locality as for preceding; Mar. 2015; R.P. Indicatti and J.P.L. Guadanucci leg.; CAD 605 • 1 \&; São Paulo, Parque Estadual do Jaraguá; 
18 Jul. 2010-31 Aug. 2012; R.P. Indicatti leg.; IBSP $171840 \bullet 6$ ô ; same collection data as for preceding; IBSP 171802, IBSP 171842 to 171843 , IBSP 171845 to $171847 \cdot 1$ ô; São Paulo, Jardim Botânico de São Paulo; 2338'30.7" S, 46³7'14.2" W; 20 Oct. 1950; Kulmann leg.; MZSP 7800 • 1 q; São Paulo, Ipiranga; $23^{\circ} 35^{\prime} 07.8^{\prime \prime} \mathrm{S}, 46^{\circ} 35^{\prime} 56.4^{\prime \prime} \mathrm{W}$; 5 Jan. 1998; MZSP 16060 • 1 \%; São Paulo, Santo Amaro;

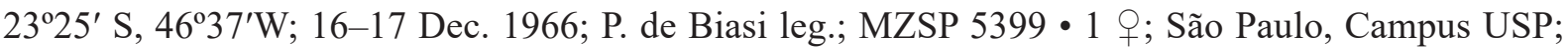
2333'34" S, 46²3'26" W; 10 Sep. 1975; O. Froelich leg.; MZSP 13886 • 1 đ̊; São Paulo, Mata do Viveiro; 1980; MZSP 19530 • 1 ग̃; São Paulo, Instituto de Pesquisas Energéticas e Nucleares; 12 Aug.

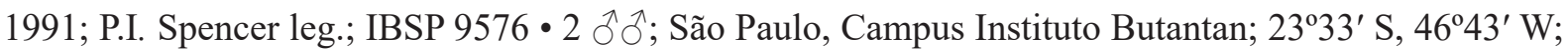
12-13 Nov. 2012; Equipe IBSP leg.; IBSP 210144 • 1 ठ̊; São Paulo, Campus Instituto Butantan; 30 Jul. 2009; F.P. Santos leg.; IBSP 210130 • 1 ठ̊; São Paulo, Campus Instituto Butantan; IBSP 9592 • 1 ठ̊; São Paulo, Campus Instituto Butantan; 20 Oct. 1988; A. Hoge leg.; IBSP 9563 • 1 đ; São Paulo, Campus Instituto Butantan; 7 Aug. 2003; D. Candiani and C.S. Fukushima leg.; IBSP 9835 • 1 §̊; São Paulo,

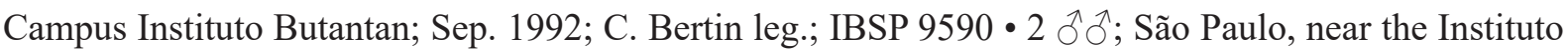

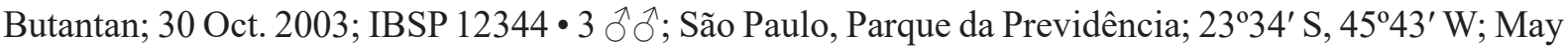
2000-Feb. 2001; D. Candiani leg.; IBSP 14398, IBSP 14742 to 14743 • 2 우; São Paulo, Parque Ilha dos Eucaliptos, Represa Guarapiranga; 2340'17" S, 4643'39" W; 7-13 Apr. 2005; I. Cizauskas leg.;

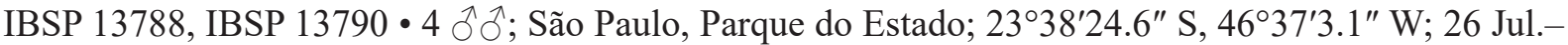

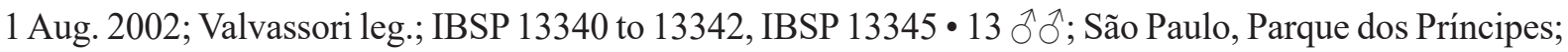

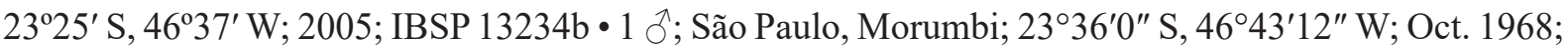

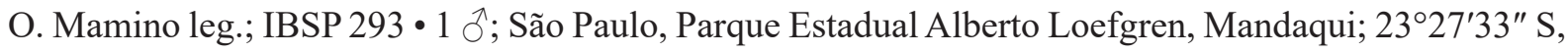
46³8'2" W; May 1973; IBSP 102438 • 1 q; highway Raposo Tavares, Km 21; 21 Jul. 1977; P. Alicke

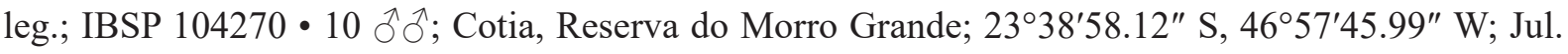

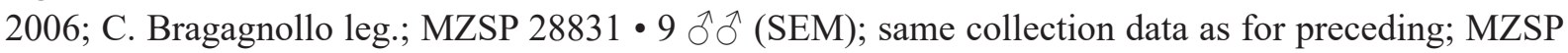

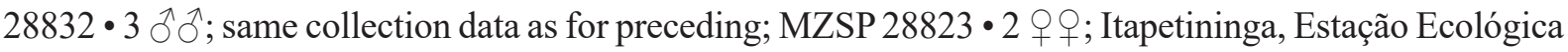
de Angatuba; 2324'57" S, 48²1'39" W; 11-16 Nov. 2002; C. Firmo et al. leg.; IBSP 258658 to 258659

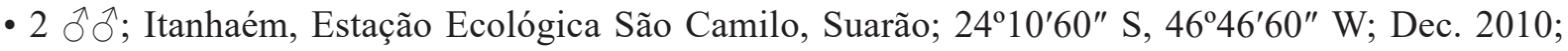
G. Aparecida and M. Pessoa leg.; IBSP 224494 to 224495 • 1 đ̊; Peruíbe, Estação Ecológica da Juréia

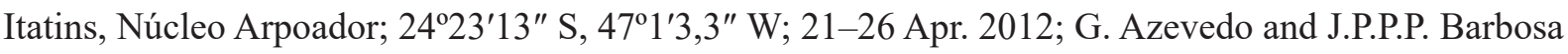

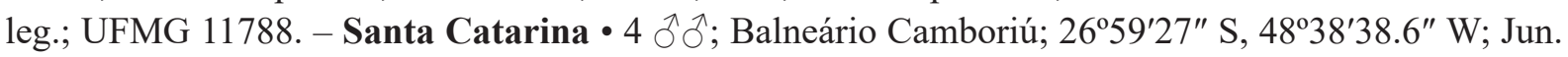
2009-Jun. 2010; M. Zucatelli leg.; IBSP 245677 to 245680.

\section{Description}

Male (MZSP 26023)

Habitus. See Fig. 11A.

Measurements. TBL 12.8, CL 7, CW 5.9, LL 0.9, LW 1.2, SL 3.4, SW 4.

CoLor. Brownish carapace and legs, sternum and coxae yellow brown (Fig. 11A-B), abdomen brown.

Prosoma. Carapace and ocular arrangement as shown in Fig. 11A. Eye tubercle: 1.6 long; 1.5 wide. Distance AME-ALE 0.9. Eye diameters: AME 0.4, ALE 0.4, PME 0.4, PLE 0.5. Thoracic fovea procurved (Fig. 11A). Labium and sternum without cuspules (Fig. 11B). Basal segment of chelicerae with a prolateral row of 9 teeth, 7 large and 2 small, and 6 small retrolateral teeth (Fig. 12A). Salient rastellum, presenting 12-13 spines of the same size (Fig. 12A-B).

LeGs. Tibia, metatarsus and tarsus I as shown in Fig. 11G. Leg I with double tibial apophysis, with wider apical branch, thickened base and conical distal area (Figs 11H-I, 12C). Pseudoscopula: tarsus I-III covered, IV absent. 

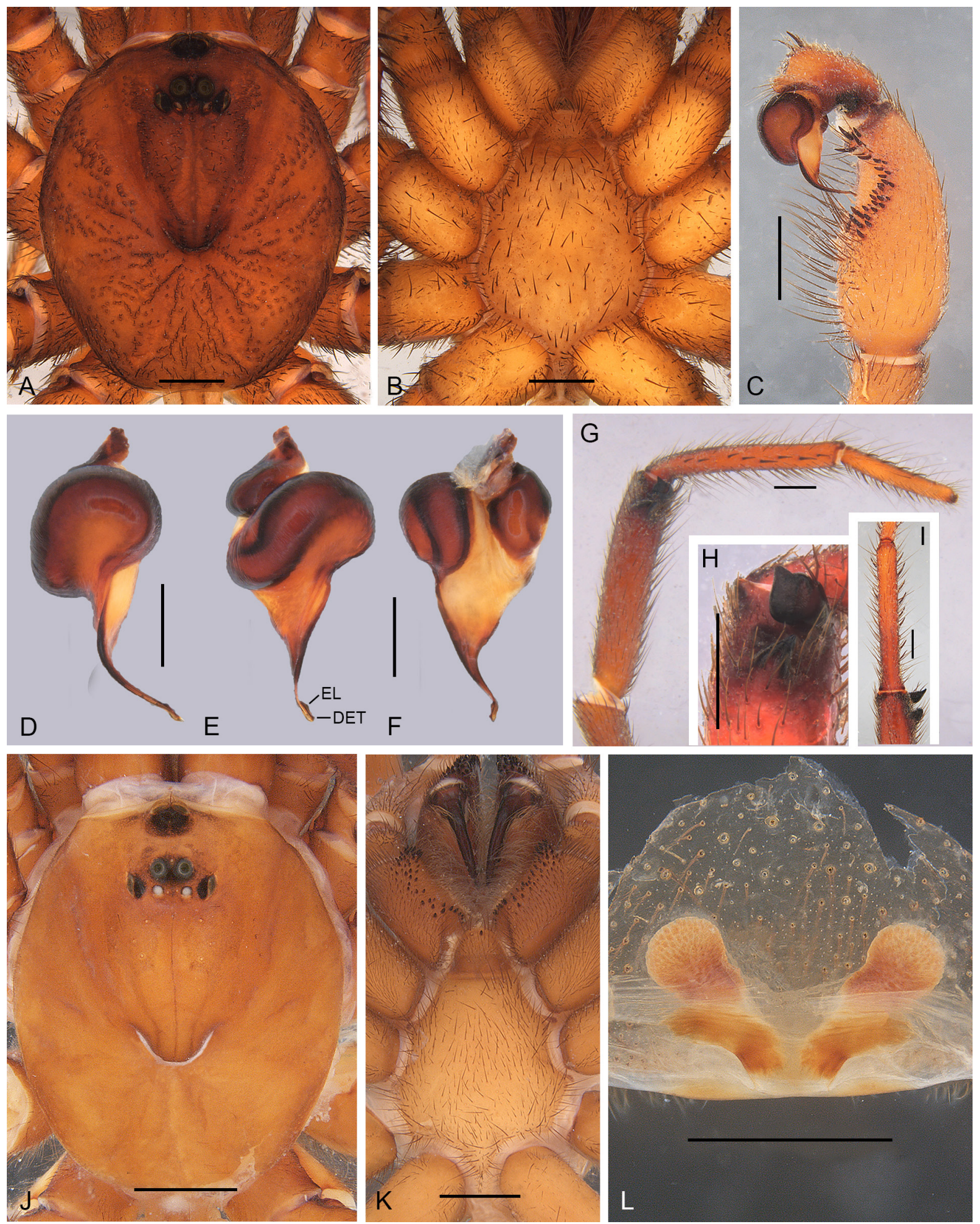

Fig. 11. Idiops camelus (Mello-Leitão, 1937). A-I. đ̂ (MZSP 26023). A. Prosoma (dorsal view). B. Prosoma (ventral view). C. Part of palp (retrolateral view). D-F. Palpal bulb. D. Dorsal view. E. Retrolateral view. F. Prolateral view. G. Tibia, metatarsus and tarsus I (prolateral view). H. Tibial apophysis (prolateral view). I. Tibial apophysis and metatarsus I (dorsal view). J-L. o (IBSP 9579). J. Prosoma (dorsal view). K. Prosoma (ventral view). L. Genitalia (dorsal view). Abbreviations: DET = distal embolar tooth; EL= embolar lamella. Scale bars: A-B, D-L $=1 \mathrm{~mm} ; \mathrm{C}=2 \mathrm{~mm}$. 
Palp. Tibia with conspicuous retrolateral depression, bordered by short spines, with the exception of the spines of the basal and apical ends, which are twice the size of the others (Fig. 11C); embolus with subapical torsion and presence of a small lamella at spermatic duct opening (Figs 11D-F, 12E-F).

PalP and leg measurements. Palp $=10.6(3.6,2,3.5,1.5), \mathrm{I}=19.7(5.9,2.8,4.4,4.3,2.3), \mathrm{II}=24$ (6.3, $2.9,5.4,6.1,3.3), \mathrm{III}=18.3(4.6,2.8,3.1,4.9,2.9), \mathrm{IV}=24.9(6.6,3,3,5.4,6.3,3.3)$.

Spination. Palp: Ti r3-3, Ta d-0-0-6. Leg I: Ti r0-1-2, Mt p5-3-2, r1-1-2, Ta p4-3-1, r3-2-2. Leg II: Ti v1-2-1-2, Mt p0-0-1, r1-1-3, Ta p0-1-2, r0-2-3. Leg III: Pa p4-5-7, r0-0- 3, Ti v0-2-0-4, p1-2-2, r0-1-3, Mt v2-3-2-3, p3-3-3, r3-1-3, Ta p1-2-5, r0-2-3. Leg IV: Pa p17-4-0, Ti v0-2-0-4, Mt v1-2-2-3, Ta v2-4-11.

\section{Female (IBSP 9579)}

Habitus. See Fig. 11J.

Measurements. TBL 18.4, CL 8.3, CW 8, LL 1.5, LW 1.8, SL 5.4, SW 4.9.

CoLOR. Brownish carapace and legs, sternum and coxae yellow brown (Fig. 11J-K), abdomen brown.

Prosoma. Carapace and ocular arrangement as shown in Fig. 11J. Eye tubercle: 2 long; 2 wide. Distance AME-ALE 1.3. Eye diameters: AME 0.4, ALE 0.6, PME 0.3, PLE 0.6. Thoracic fovea procurved (Fig. 11J). Labium with 2-5 cuspules (Fig. 11K). Maxilla with 48 cuspules, distributed over the anterior ventral half (Fig. 11K). Basal segment of chelicerae with a prolateral row of 6 large teeth and a retrolateral row with 4 small teeth.
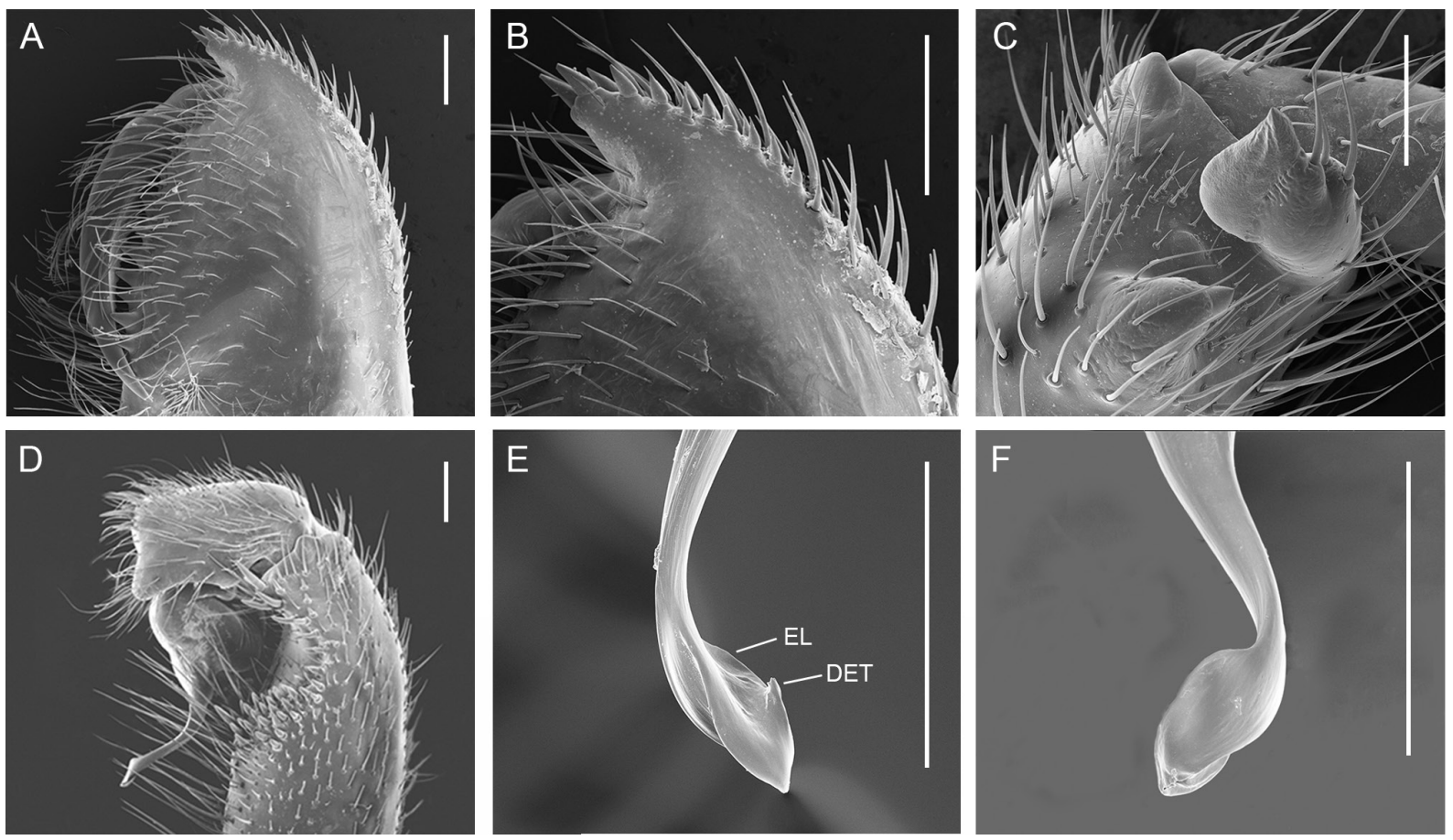

Fig. 12. Scanning electron micrographs of Idiops camelus (Mello Leitão, 1937), đ̊ (MZSP 28832). A. Chelicera (retrolateral view). B. Rastellum (retrolateral view). C. Tibial apophysis (dorsal view). D. Part of the palp, showing retrolateral depression. E-F. Detail of the distal portion of the embolus. E. Retrolateral view. F. Prolateral view. Abbreviations: DET = distal embolar tooth; EL = embolar lamella. Scale bars $=0.5 \mathrm{~mm}$. 
Palp and Leg measurements. Palp $=14.3(4.8,3.2,3.1,3.2), \mathrm{I}=16.7(5.4,3.7,3.6,2.8,1.2), \mathrm{II}=14.9$ $(4.9,3.4,3,2.3,1.3), \mathrm{III}=15.6(4.3,3.7,2.4,3.1,2.1), \mathrm{IV}=21.6(6.1,4.4,4.5,4.3,2.3)$.

Sination. Palp: Fe p0-0-1, Pa p0-0-1, Ti p9-6-12, Ta p14-11-10. Leg I: Ti p7-7-10, r8-7-13, Mt p11-9-7, r12-8-13, Ta v0-0-5, p6-5-3, r6-4-3. Leg II: Ti p4-4-7, r2-1-7, Mt p8-4-2, r12-8-10, Ta v0-0-9, p6-1-2, r6-5-3. Leg III: Pa p5-5-17, r0-1-4, Ti v1-1-1, p4-5-12, r1-3-10, Mt v1-2-2, p11-3-3, r8-2-1, Ta v0-1-4, p0-1-3, r0-0-1. Leg IV: Pa p65-7-0, Ti v2-1-3, Mt 1-3-2, p0-1-0, Ta v1 -5-11.

SPERMATHECAE. Receptacula with evident granules. Duct with sclerotized apical portion (Fig. 11L).

\section{Distribution.}

Brazil. Mainly distributed in Atlantic Forest phytophysiognomies, with records for Southeast region (center-south of Minas Gerais, Rio de Janeiro and São Paulo) and northeast of Santa Catarina (Fig. 3D).

Idiops carajas Fonseca-Ferreira, Zampaulo \& Guadanucci, 2017

Figs 3B, 6D-E, 13-14

Idiops carajas Fonseca-Ferreira, Zampaulo \& Guadanucci, 2017: 191, figs 29-38.

\section{Emended diagnosis}

The male of Idiops carajas (Figs 13A-I, 14) differs from the other Neotropical species, except I. petiti, by the palpal tibia having spines concentrated on the basal half of the retrolateral depression (Figs 13C, 14D), apophysis with a narrow rectangular apical branch (Figs 13G-H, 14C) and by the presence of a lateral lamella that extends along the median portion of the embolus (Figs 13E-F). Differs from I. petiti by having the subapical portion of the embolus thickened in dorsal view (Fig. 13D) and the arrow-shaped apical end in retrolateral view (Figs 13E-F, 14E). Females (Fig. 13J-L) are distinguished from congeners, except I. petiti, by having the spermathecae with a sclerotized trapezoidal base. Differs from I. petiti by the ducts having a thickened basal half and shorter apical half and by the rounded receptacles, separated from the ducts by a strong constriction (Fig. 13L).

\section{Type material}

\section{Holotype}

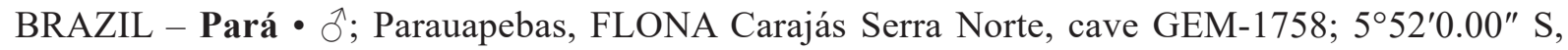
4952'60.00" W; 23 Nov. 2010; R.A. Zampaulo leg.; IBSP 166619.

\section{Paratype}

BRAZIL - Pará • 1 q; same collection data as for holotype; IBSP 166620.

\section{Other material examined}

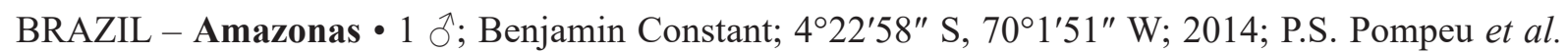
leg.; IBSP 243963. - Pará • 1 ô (SEM); Paraoapebas, Serra Norte, Serra de Carajás; 552' S, 4953’ W; 29 Mar.-6 Apr. 1989; N. Degallier leg.; MPEG 0109• 1 đ̊; Flona Carajás, Cave N3-033; 66'34.92" S, 50¹1'40.11" W ; 5-17 Mar. 2013; Equipe Carste leg.; IBSP 174029 • 1 đ̊; Melgaço, Igarapé do Laranjal; $1^{\circ} 48^{\prime 2} 21.44^{\prime \prime}$ S, 5043'0" W; 7 Apr. 1998; J.A.R. Bernardi and R.A.J. Rocha leg.; MPEG $0111 \bullet 1$ đ;; Almeirim, Rio Jari; 0041'25.93" S, 5249'9.21" W; 17-23 Aug. 2004; T. Gardner leg.; MPEG 7592

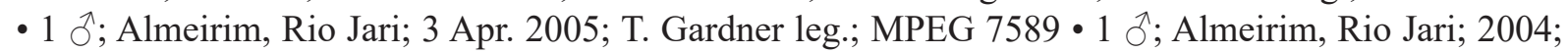
T.C.S. Avila Pires leg.; MPEG 7591 • 2 $\widehat{\jmath}$; Almeirim, Rio Jari; 22 Mar. 2005; T. Gardner \& M.A. Ribeiro Junior leg.; MPEG 7587, MPEG 7590 • 1 ð’; Tucuruí, Base IV; 346'4" S, 4940'22" W; 8-22 Feb. 1980; T. Gardner leg.; MPEG 0115. - Mato Grosso • $1 \partial^{\lambda}$; Chapada dos Guimarães; $15^{\circ} 27^{\prime} 39^{\prime \prime}$ S, $55^{\circ} 45^{\prime} 0^{\prime \prime}$ W; 

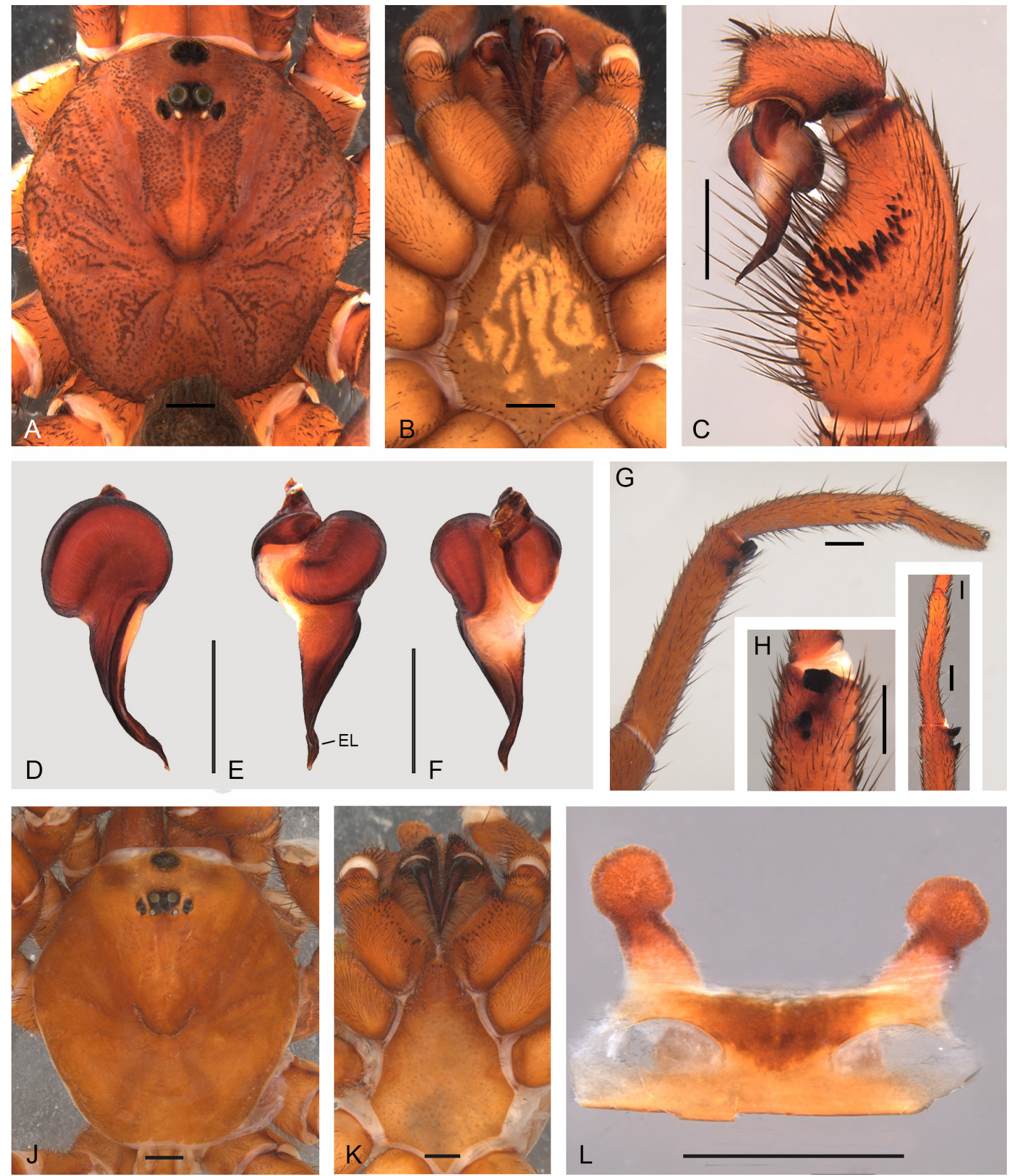

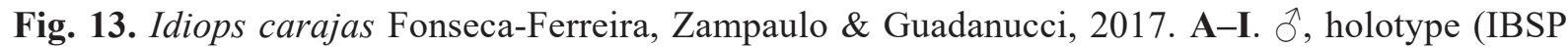
166619). A. Prosoma (dorsal view). B. Prosoma (ventral view). C. Part of palp (retrolateral view). D-F. Palpal bulb. D. Dorsal view. E. Retrolateral view. F. Prolateral view. G. Tibia, metatarsus and tarsus I (prolateral view). H. Tibial apophysis (prolateral view). I. Tibial apophysis and metatarsus I (dorsal view). J-L. +, paratype (IBSP 166620). J. Prosoma (dorsal view). K. Prosoma (ventral view). L. Genitalia (dorsal view). Abbreviation: $\mathrm{EL}=$ embolar lamella. Scale bars $=1 \mathrm{~mm}$. 


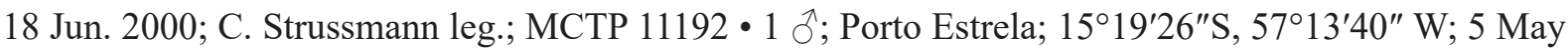
2019; J.R. Lema, D. Castro and M. Pessoa-Silva leg.; IBSP249141. - Mato Grosso do Sul • 1 đ; Corumbá, Morro Santa Cruz; 19¹2'07.6" S, 57²36'09.9" W; Jun. 2003; V.L. Ferreira leg.; MCTP 17591. Tocantins • $10^{\top}$; Ananás; 6 $13^{\prime} 34.70^{\prime \prime}$ S, 48²5'2.39" W; 21 Apr. 2009; W.U. Oliveira and M.D. Miranda leg.; UFMG 5749.

\section{Emended description}

Male and female recently described by Fonseca-Ferreira et al. (2017). New data on the male and female are included.

\section{Male (holotype IBSP 166619)}

Prosoma. Basal segment of chelicerae with a prolateral row of 9 teeth, 6 large and 3 small, and 5 small retrolateral teeth (Fig. 14A); salient rastellum, presenting 12-13 short, thick spines with larger distal ends (Fig. 14B).

\section{Female (paratype IBSP 166620)}

Prosoma. Basal segment of chelicerae with a prolateral row of 9 large teeth and 5 small retrolateral teeth; robust rastellum, presenting approximately 25 short and thick spines on a tubercle.

\section{Distribution}

Brazil. Distributed in the phytophysiognomies of Amazon and Cerrado, with records from the west of Central-west region (Mato Grosso, Mato Grosso do Sul) and from the North region (Amazonas, Pará and Tocantins) (Fig. 3B).
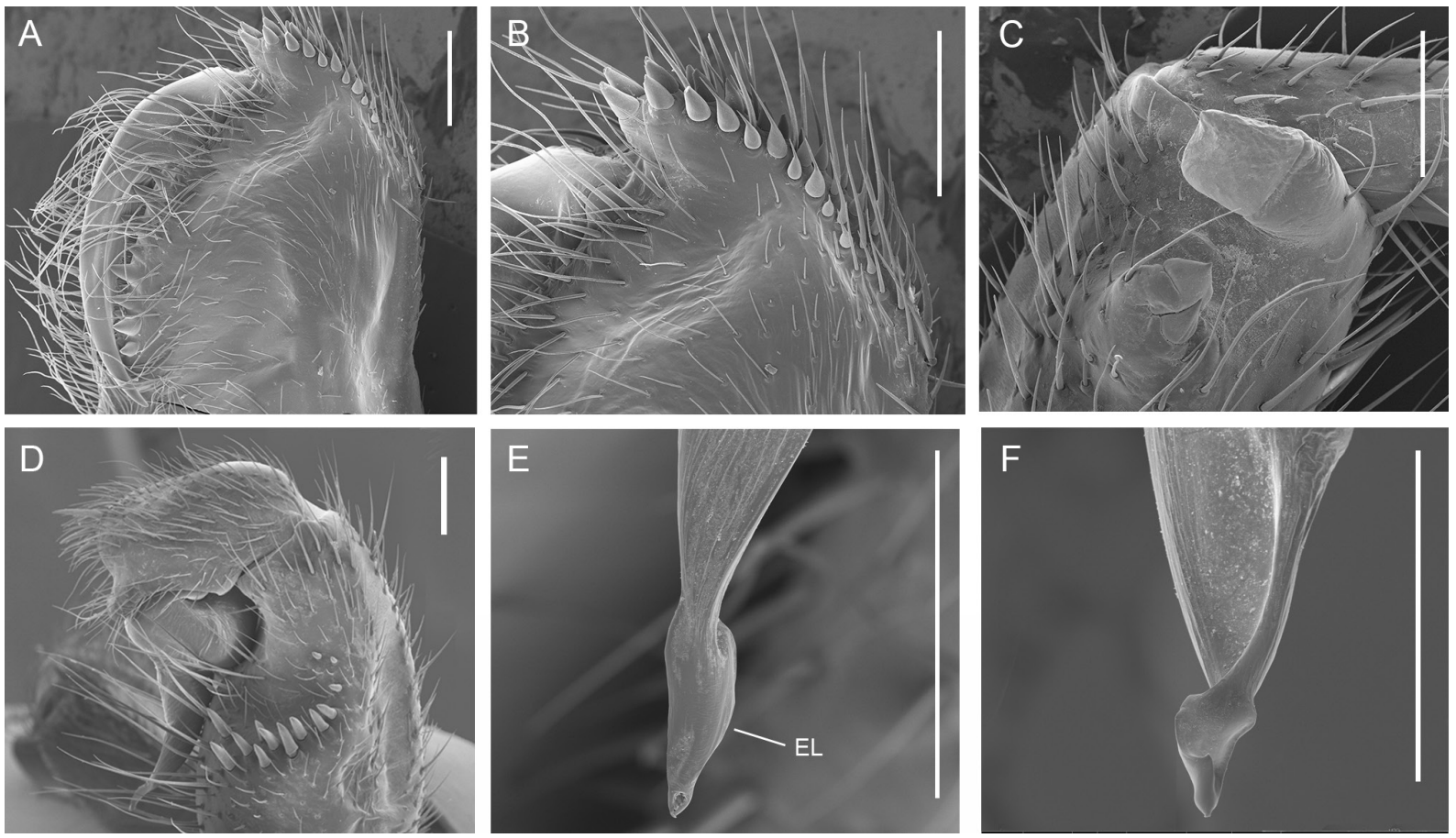

Fig. 14. Scanning electron micrographs of Idiops carajas Fonseca-Ferreira, Zampaulo \& Guadanucci, 2017, $\widehat{o}$ (MPEG 0109). A. Chelicera (retrolateral view). B. Rastellum (retrolateral view). C. Tibial apophysis (dorsal view). D. Part of the palp, showing retrolateral depression. E-F. Detail of the final portion of the embolus. E. Retrolateral view. F. Prolateral view. Abbreviation: EL = embolar lamella. Scale bars $=0.5 \mathrm{~mm}$. 
Idiops clarus (Mello-Leitão, 1946)

Figs 3C, 6B, 15-16

Juambeltzia clara Mello-Leitão, 1946: 6, figs 6-9.

Juambeltzia clara - Bücherl 1957: 383, figs 3-3a.

Idiops clarus - Schiapelli \& Gerschman de Pikelin 1971: 58, figs 1-7. — Ferretti et al. 2017: 977, figs $1 \mathrm{a}-\mathrm{f}, 2 \mathrm{a}-\mathrm{c}, 3 \mathrm{~d}$.

\section{Emended diagnosis}

Males (Fig. 15A-H) and females (Fig. 15I-K) of Idiops clarus differ from the other Neotropical species by having the posterior lateral eyes separated from the median eye group (Fig. 15A, I) and by the rounded sternum (Fig. 15B). Males also differ from others by having the palpal tibia short and thick, with a projection at the base of the retrolateral depression (also present in I. fuscus and I. pirassunsunguensis) (Fig. 15C) and by the presence of a distal embolar tooth close to the opening of the sperm duct (also present in I. germaini) (Fig. 16E). Females differ by the aspect of the spermathecae with the extensive basal area divided by a non-sclerotized portion and by ducts distant from each other and a sclerotized area in the transition between duct and receptacle (Fig. 15K).

\section{Type material}

\section{Holotype}

URUGUAY - Florida • ${ }^{\top}$; San Gabriel; A. Juambeltz leg.; MHNM. The type specimen was not found in the MHNM, probably lost.

\section{Other material examined}

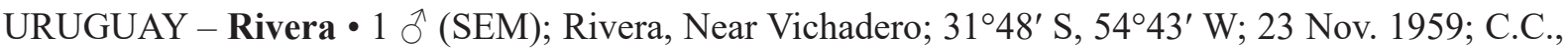

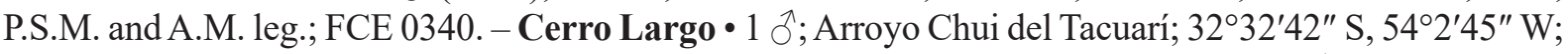
15 Nov. 1972; A.S.P., C.C., L.Z.C. and N.E.L. leg.; MNHN 295. - Río Negro • 1 đ’; Tres Arboles; $32^{\circ} 26^{\prime} \mathrm{S}, 56^{\circ} 42^{\prime} \mathrm{W}$; 1 Apr. 2009; Stora Enso/Trampa PC; FCE $1075 \bullet 1$ O’; Tres Arboles; 1 Apr. 2009; Stora Enso/Trampa PB2; FCE 1085 • 2 đ̊ ô;; Tres Arboles; 1 Mar. 2009; Stora Enso/Trampa PC; FCE 1115

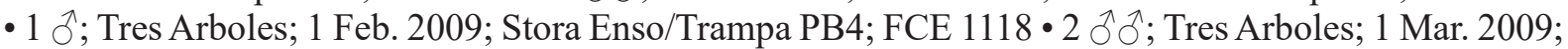
Stora Enso/Trampa ECD; FCE 1152 • 1 đ; Tres Arboles; 1 Mar. 2009; Stora Enso/Trampa PB; FCE 1161

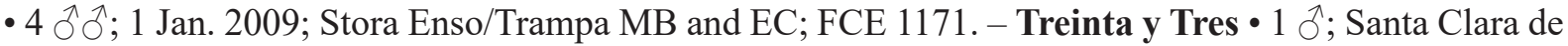
Olimar; 32 $2^{\circ} 5^{\prime}$ S, 54 58 ${ }^{\prime}$ W; 12 Jan. 1960; L.C. Zolessi and A. Spiritoso leg.; FCE 0337. - Lavalleja •

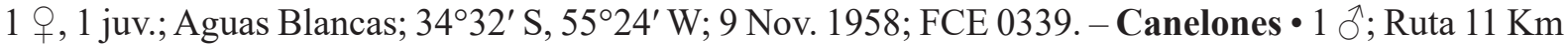

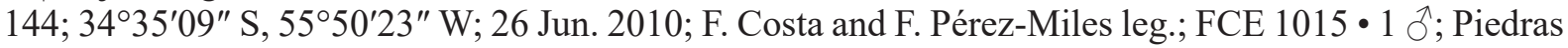

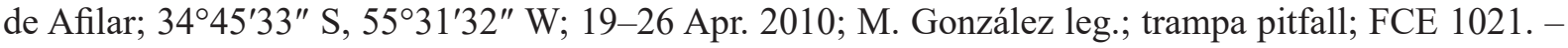

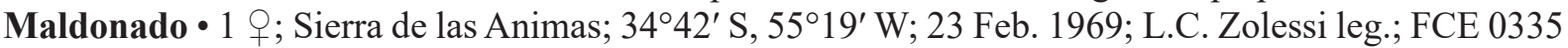

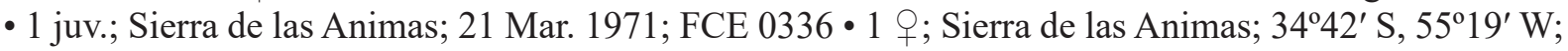
21 Mar 1971; FCE 0338 • 2 ㅇ 9 , 2 juvs; Sierra de las Animas; 2 Nov. 1969; L.C. Zolessi leg.; FCE 0342 - 1 क; Sierra de las Animas; 11 Feb. 1970; L.C. de Zolessi leg.; MNHN 373 • 1 đ̊; Sierra de las Animas; 21 Mar. 1971; S. Etcheverrigaray leg.; MNHN 718. - Montevideo • 1 đ̊̄; Prado, Liceo Bauzá; 3451' S, 56 ${ }^{\circ} 12^{\prime}$ W; 19 Apr. 2002; A. Mignone leg.; FCE 0341.

The specimens registered for Argentina and reviewed by Ferretti et al. 2017 were not examined here. Despite this, they were included in the species distribution map.

\section{Description}

Male (FCE 0341)

Habitus. See Fig. 15A.

Measurements. TBL 11, CL 5.7, CW 4.8, LL 0.6, LW 1.2, SL 3, SW 2.9. 
CoLor. Carapace, legs and sternum light brown (Fig. 15A-B), abdomen gray.

Prosoma. Carapace and ocular arrangement as shown in Fig. 15A. Eye tubercle: 1.4 long; 1.6 wide. Distance AME-ALE 0.8. Eye diameters: AME 0.3, ALE 0.4, PME 0.3, PLE 0.3. Thoracic fovea procurved. Labium and sternum without cuspules (Fig. 15B). Basal segment of chelicerae with a prolateral row of
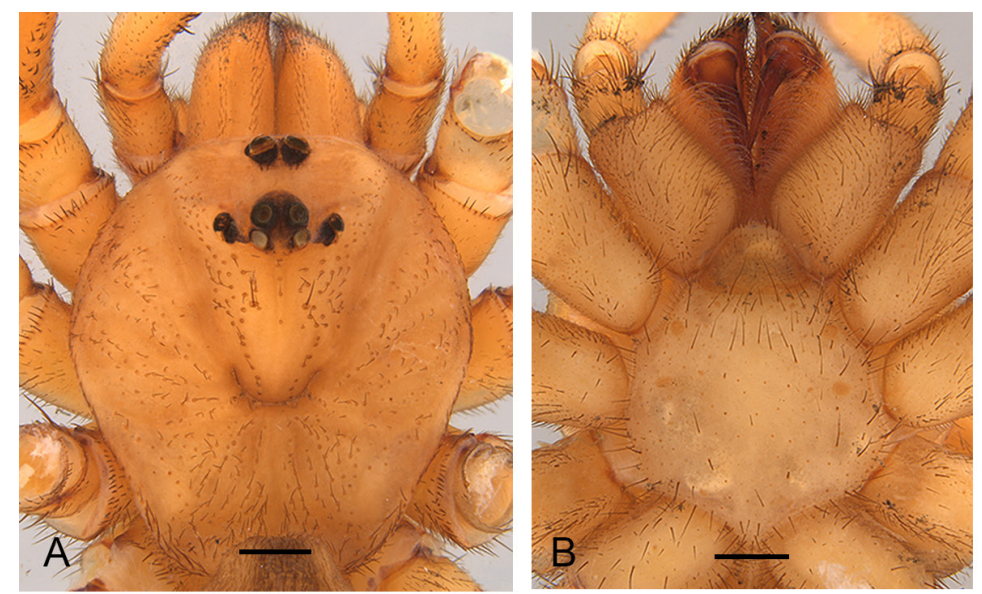
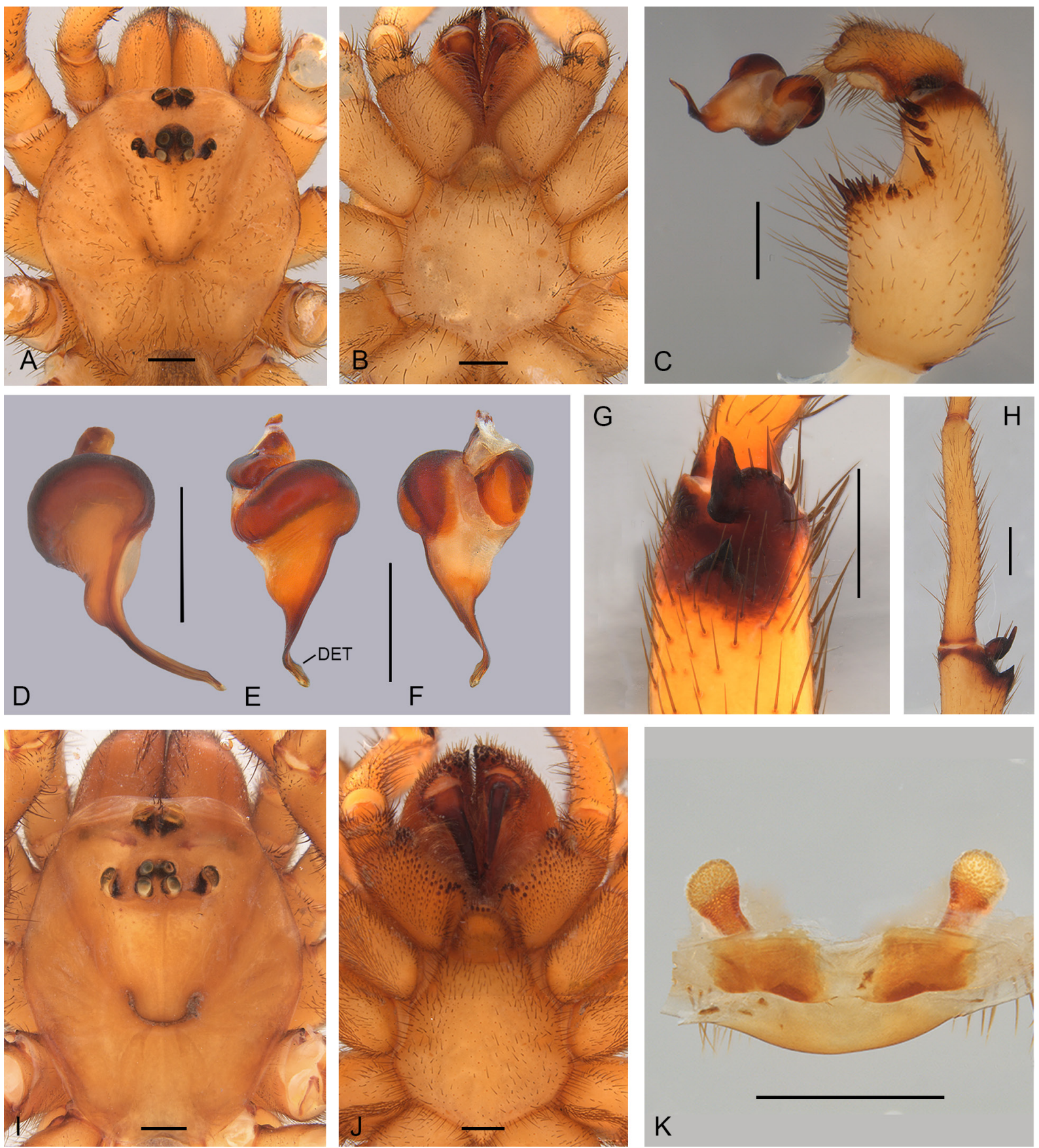

Fig. 15. Idiops clarus (Mello-Leitão, 1946). A-H. ô (FCE 0341). A. Prosoma (dorsal view). B. Prosoma (ventral view). C. Part of palp (retrolateral view). D-F. Palpal bulb. D. Dorsal view. E. Retrolateral view. F. Prolateral view. G. Tibial apophysis (prolateral view). H. Tibial apophysis and metatarsus I (dorsal view). I-K. ㅇ, (FCE 0338). I. Prosoma (dorsal view). J. Prosoma (ventral view). K. Genitalia (dorsal view). Abbreviation: $\mathrm{DET}=$ distal embolar tooth. Scale bars $=1 \mathrm{~mm}$. 
5 large teeth and retrolateral row with 9 small teeth (Fig 15A), rastellum with 8 spines, distal spines are longer (Fig. 16B).

LEGS. Tibia and metatarsus I as shown in Fig. 15H. Leg I with an apical branch of tibial apophysis with a short and triangular basal spine, and an apical branch with a conical spine twice the size of basal spine. Pseudoscopula: tarsus I divided into rows of thick hair; tarsus II-IV totally covered.

PALP. Tibia with conspicuous retrolateral depression, with projection on basal portion and larger spines concentrated on basal and apical portions (Figs 15G, 16C); embolus with subapical torsion and presence of a small embolar tooth close to spermatic duct opening (Figs 15D-F, 16E-F).

Palp and leg measurements. Palp $=9.3(3,1.6,3.1,1.6), \mathrm{I}=21.3(5.9,3.2,4.3,5,2.9), \mathrm{II}=19.7$ (5, $2.5,3.4,4.2,2.6), \mathrm{III}=14.9(3.5,2.3,2.3,4,2.8), \mathrm{IV}=21.2(5.4,2.9,4.5,5.4,3)$.

Sination. Palp: Ti r24, Ta d0-0-4. Leg I: Fe d1-1-2, Ti v1r-1r-2r, Mt 1r-2r-1ap, Ta r2-1-0. Leg II: Fe d1-1-2, Ti v0-2r-1r-1, Mt v1r-1r-1r-2, Ta p0-0-1, r2-2-0. Leg III: Fe d1-1-1, Pa d1-1-0, p3-5-8, r0-0-2, Ti v0-1 r-0-2, p1-1-4, r1-1-2, Mt v3-4-0-4, p3-2-2, r0-1-2, Ta p0-4-8, r0-4-8. Leg IV: Fe d1-1-2, Pa p16-3-0, Ti v1-2-2, Mt v1-2 -1-3, Ta p2-3-6, r0-0-2.

Female (FCE 0338)

Habitus. See Fig. 15I.

Measurements. TBL 17.3, CL 7.5, CW 6.3, LL 1.2, LW 1.6, SL 4.4, SW 4.3.

CoLoR. Carapace, legs and sternum light brown (Fig. 15I-J), abdomen gray.
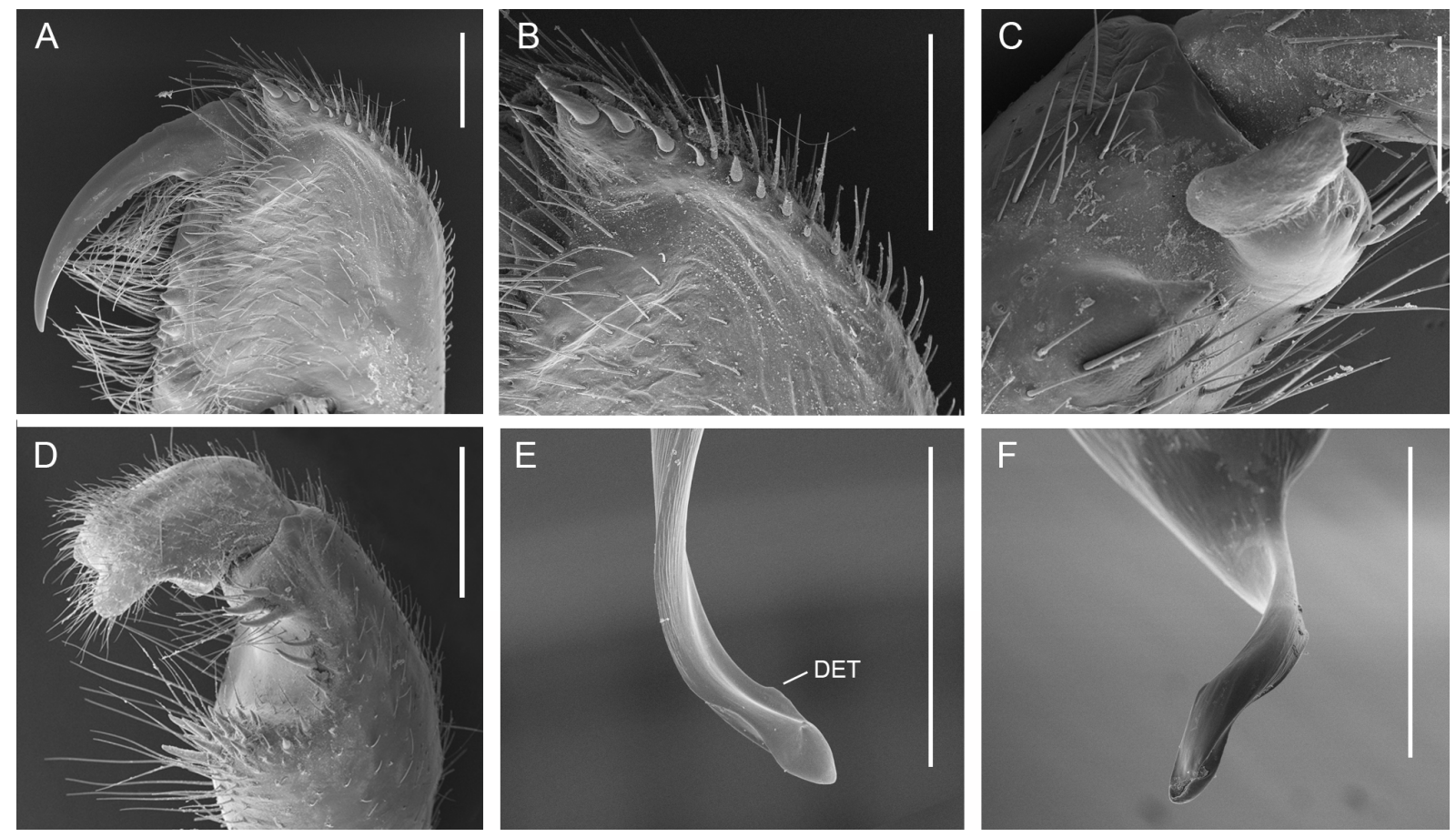

Fig. 16. Scanning electron micrographs of Idiops clarus (Mello-Leitão, 1946), $\widehat{\jmath}$ (FCE 0340). A. Chelicera (retrolateral view). B. Rastellum (retrolateral view). C. Tibial apophysis (dorsal view). D. Part of the palp, showing retrolateral depression. E-F. Detail of the final portion of the embolus. E. Retrolateral view. F. Prolateral view. Abbreviation: $\mathrm{DET}=$ distal embolar tooth. Scale bars $=0.5 \mathrm{~mm}$. 
Prosoma. Carapace and ocular arrangement as shown in Fig. 15I. Eye tubercle: 2.2 long; 2.7 wide. Distance AME-ALE 1. Eye diameters: AME 0.4, ALE 0.6, PME 0.5, PLE 0.7. Thoracic fovea procurved. Labium with 4-6 cuspules (Fig. 15J). Maxilla with 83 cuspules, distributed over the anterior ventral half (Fig. 15J). Basal segment of chelicerae with a prolateral row of 5 large teeth and a retrolateral row with 7 small teeth, grouped at basal half.

PalP And leg measurements. Palp = $12.2(4.1,2.6,2.7,2.8), \mathrm{I}=13.3(4.2,3,2.9,2.2,1)$, II $=11.1$ (3.4, $2.7,2.3,2,1), \mathrm{III}=12(3.3,2.6,1.7,2.4,2), \mathrm{IV}=15.8(4.1,3.4,3.3,3.2,1.8)$.

Sination. Palp: Fe p0-0-2, Pa p0-0-1, Ti p7-8-11, r3-10-13, Ta v0-0-6, p12-10-8, r12-10-12. Leg I: Ti p5-5-8, r6-8-12, Mt p11-8-10, r9-8-7, Ta v0-6-7, p4-5-2, r5-4-3. Leg II: Ti p2-7-6, r2-4-1, Mt p10-9-7, r4-5-3, Ta v0-1-7, p4-5-3, r4-2-1. Leg III: Pa p5-5-16, r0-0-2, Ti p5-7-10, r3-7-10, Mt v0-0-2, p12-7-6, r7-5-4, Ta v0-6-22. Leg IV: Pa p30-5-0, Ti v0-1-1-2, Mt v0-2-5-3, Ta v1-9-14.

Spermathecae. Duct with basal portion shorter than apical portion and non-sclerotized spherical receptacles with evident granules (Fig. 15K).

\section{Distribution}

Uruguay and Argentina (Fig. 3C).

Idiops duocordibus Fonseca-Ferreira, Guadanucci \& Brescovit sp. nov. urn:lsid:zoobank.org:act:D3387D49-8B8B-42C0-ADB9-88D5A8A78B62

Figs $3 \mathrm{~B}, 17 \mathrm{~A}-\mathrm{L}$

\section{Diagnosis}

Males and females of Idiops duocordibus sp. nov. differ from the other Neotropical species by the light brown coxae and trochanters, contrasting with the brown body (Fig. 17A-L). The male resembles that of I. rohdei by the short metatarsus I with prolateral curvature and with a slight prolateral projection on the apical half (Fig. 17I), but differs by the tibial apophysis with a triangular apical branch twice the size of the basal branch (Fig. 17G-H) and by the weakly curved embolus (Fig. 17D). The female (Fig. 17J-L) differs from those of the other species by having the spermathecae with bilobed receptacles in the shape of a heart (Fig. 17L).

\section{Etymology}

The specific epithet refers to the heart-shaped receptacles of the female genitalia.

\section{Type material}

\section{Holotype}

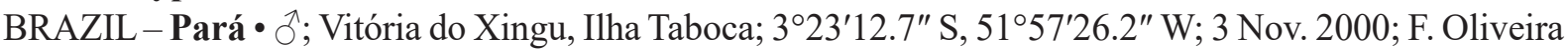
leg.; MPEG 0124.

\section{Paratypes}

BRAZIL - Pará • $1 \partial^{\lambda}$; Almeirim, Reserva de Itapeguara; $0^{\circ} 32^{\prime} 04.9^{\prime \prime}$ S, 5248'14.8" W; Dec. 2001; MPEG 0122 • 1 q; Arapari, Rio Tocantins, left bank; 452'23.6" S, 49³1'39.5” W; 14 Mar. 1984; W.L. Overal leg.; MPEG 0123.

\section{Other material examined}

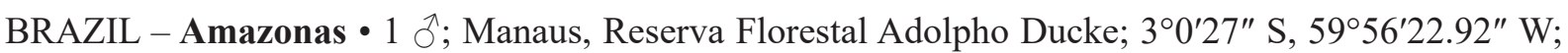
9 Sep. 1991; H. Höfer and T. Gasnier leg.; INPA 4592 • 1 đ̊; Manaus, Reserva Florestal Adolpho Ducke; 
14-24 Jul. 1991; A.D. Brescovit leg.; MCN 21485. - Pará • 2 ठో đ̊; Vitória do Xingu, Ilha Taboca; 3²3'12.7" S, 5157'26.2" W; 23 Nov. 2000; F. Oliveira leg.; MPEG 0112, MPEG 0114.

\section{Description}

Male (holotype MPEG 0124)

Habitus. See Fig. 17A.

Measurements. TBL 10, CL 4.6, CW 4, LL 0.5, LW 0.7, SL 2.4, SW 2.2.

CoLOR. Carapace brown, light brown coxae and trochanter (Fig. 17A-B). Abdomen dark gray.

Prosoma. Carapace and ocular arrangement as shown in Fig. 17A. Eye tubercle: 0.68 long; 1.08 wide. AME-ALE distance 0.63. Eye diameters: AME 0.32, ALE 0.34, PME 0.13, PLE 0.22. Thoracic fovea procurved (Fig. 17A). Labium and sternum without cuspules (Fig. 17B). Basal segment of chelicerae with a prolateral row of 5 small teeth equally distributed, rastellum with 5 spines (Fig. 17B).

LEGs. Tibia, metatarsus and tarsus I as shown in Fig. 17G. Leg I with double tibial apophysis. Leg I tibial apophysis with apical branch twice the size of basal branch and with a conspicuous spine (Fig. 17G-I). Pseudoscopula: tarsus I weakly covered (Fig. 17G), tarsus II-IV totally covered.

Palp. Tibia with thickened median portion and with larger spines concentrated in basal portion of retrolateral depression (Fig. 17C), embolus elongated, tapered, and slightly curved, with torsion on apical portion (Fig. 17D-F), keel along embolus length extending to apical portion (Fig. 17D).

PalP AND Leg Measurements. Palp $=6.9(2.3,1.3,2.4,0.9), \mathrm{I}=13.4(4.3,1.9,3,2.9,1.3), \mathrm{II}=11.3(3.5$, $1.8,2.5,2.5,1), \mathrm{III}=10(2.7,1.5,1.9,2.6,1.3), \mathrm{IV}=13.6(3.9,1.9,3.5,3.1,1.2)$.

SPINATION. Palp: Ti r39, Ta d0-0-3. Leg I: Pa v0-1-2, r1-2-3, Ti v1-2-3, r1-2-6, Mt v1-4-7, p0-0-2, p0-1-1, r4-4-5, Ta v0-1-0, p0-2-3, p1-2-3, r4-4-3. Leg II: Pa v0-0-3, d0-0-1, Ti v2-3-7, d3-4-8, Mt v4-6-8, d5-6-7, p1-2-4, r0-2-3, Ta p1-3-3, r2-2-3. Leg III: Pa v0-0-2, Ti v1-2-3 r0-1-0, Mt v3-6-7, d0-0-2, p2-2-3, r1-2-4, Ta p3-4-4, r1-3-3. Leg IV: Pa v0-0-3, Ti v1-2-2, Mt v1-4-8, p0-0-1, r0-2-3, Ta v2-4-5, p0-1-2, r2-3-2.

Female (paratype MPEG 0123)

Habitus. See Fig. 17J.

Measurements. TBL 9.9, CL 5.5, CW 4,8, LL 0.9, LW 1.2, SL 3.4, SW 2.9.

CoLOR. Brown carapace and trochanter, remainder of leg light brown (Fig. 17J-L). Abdomen dark gray.

Prosoma. Carapace and ocular arrangement as shown in Fig. 17J. Eye tubercle: 0.7 long; 1.1 wide. AME-ALE distance 0.9. Eye diameters: AME 0.3, ALE 0.3, PME 0.2, PLE 0.4. Thoracic fovea procurved (Fig. 17J). Labium with with 13 cuspules (Fig. 17K). Maxilla with 117 cuspules, distributed throughout the maxillae, with larger spines on anterior half(Fig. 17K). Basal segment of chelicerae with a prolateral row of 6 large teeth and 2 retrolateral small teeth, grouped in basal half, rastellum with 16-18 short and thick spines (Fig. 17K).

PalP AND Leg measurements. Palp $=6.4(2.3,1.2,1.6,1.3), \mathrm{I}=7.4(2.5,1.5,1.6,1,0.8), \mathrm{II}=6.5(2.3$, $1.6,1.2,0.9,0.5), \mathrm{III}=7(1.9,1.6,1.3,1.4,0.8), \mathrm{IV}=9.5(2.6,1.7,2.1,2.2,0.9)$.

Sination. Palp: Pa p0-0-1, Ti p4-7-7, r4-6-8, Ta p10-8-7, r10-7-9. Leg I: Ti p4-4-5, r3-5-8, Mt p8-7-8, r5-5-6, Ta p3-4-2, r5-4-0. Leg II: Ti v1-1-2, p3-3-4, r0-1-3, Mt v1-1-2, p6-7-9, r2-2-1, Ta p2-3-3, r2-1-0. 
Leg III: Pa p4-5-10, Ti v0-0-2, p2-4-6, r4-4-6, Mt v1-1-2, d0-0-2, p3-3-3, r0-2-1. Leg IV: Ti v1-1-2, Mt v1-1-3, vp0-0-2, Ta v0-1-2, p1-1-2.

SPERmatheCAE. Single sclerotized base that is divided into two divergent ducts, receptacula strongly sclerotized (Fig. 17L).
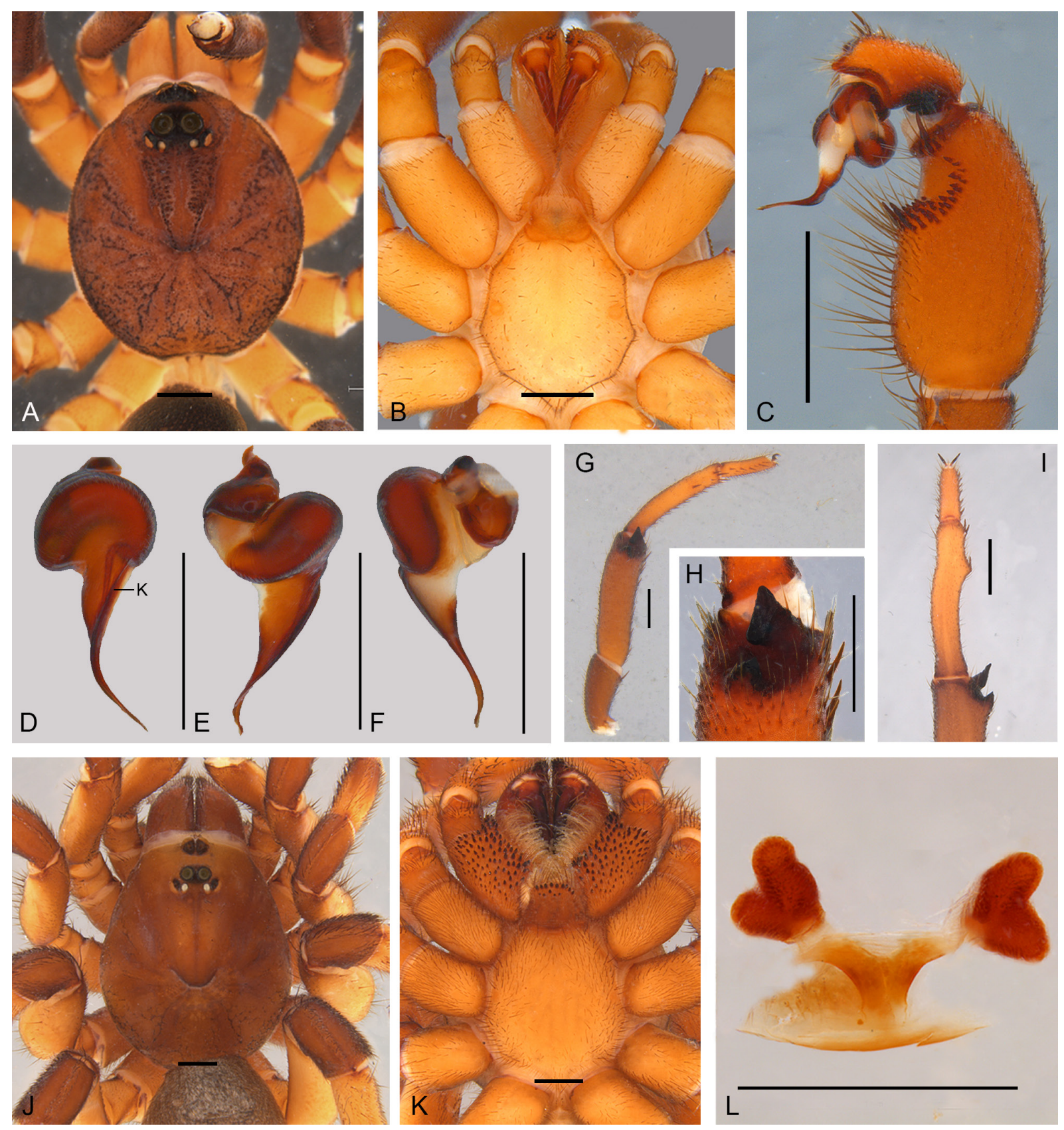

Fig. 17. Idiops duocordibus Fonseca-Ferreira, Guadanucci \& Brescovit sp. nov. A-I. ふૈ, holotype (MPEG 0124). A. Prosoma (dorsal view). B. Prosoma (ventral view). C. Part of palp (retrolateral view). D-F. Palpal bulb. D. Dorsal view. E. Retrolateral view. F. Prolateral view. G. Tibia, metatarsus and tarsus I (prolateral view). H. Tibial apophysis (prolateral view). I. Tibial apophysis and metatarsus I (dorsal view). J-L. q, paratype (MPEG 0123). J. Prosoma (dorsal view). K. Prosoma (ventral view). L. Genitalia (dorsal view). Abbreviation: $\mathrm{K}=$ keel. Scale bars $=1 \mathrm{~mm}$. 


\section{Distribution}

Brazil. Distributed in phytophysiognomies in the Amazon, with records for the North region (western Amazonas and northwestern Pará) (Fig. 3B)

Idiops germaini Simon, 1892

Figs 3D, 5B, 18-19

Idiops germaini Simon, 1892: 92.

Idiops germaini - Mello-Leitão 1923: 47. — Fukami \& Lucas 2005: 6, figs 8-10.

\section{Diagnosis}

Males of Idiops germaini differ from those of other Neotropical species of Idiops by the long and slender palpal tibia, with the retrolateral depression restricted to the apical portion (Figs 18C, 19D), and by having the tibial apophysis reduced to two long spines inserted on a low tubercle (Figs 18G-I, 19C). Females with spermathecae composed of short ducts and fully sclerotized receptacles, almost twice the diameter of the duct (Fig. 18L).

\section{Type material}

\section{Holotype}

BRAZIL - Rio de Janeiro • O’; Rio de Janeiro; P. Germain leg.; MNHN 417.

\section{Paratype}

BRAZIL - Rio de Janeiro・ 1 +; same collection data as for holotype; MNHN 9977.

\section{Other material examined}

BRAZIL - Rio de Janeiro • 1 đ (SEM); Nova Iguaçu, Parque Municipal de Nova Iguaçu; 22 $45^{\prime} 35^{\prime \prime}$ S, $43^{\circ} 27^{\prime} 6^{\prime \prime}$ W; 20 May 2004; R.L.C. Baptista leg.; MNRJ • 1 کో; Nova Iguaçu, Parque Municipal de Nova Iguaçu; 20 Jun. 2004; R.L.C. Baptista leg.; MNRJ 1 ô; Nova Iguaçu, Parque Municipal de Nova Iguaçu; Jan. 2001; R.L.C. Baptista leg.; MNRJ • 1 q; Nova Iguaçu, Parque Municipal de Nova Iguaçu; 22 Jan. 2005; D.R. Pedroso leg.; MNRJ • 1 \&; Paraty, Parque Nacional da Serra da Bocaina, Trilha da Cachoeira

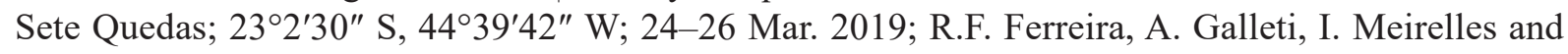

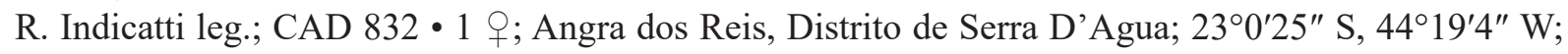
25 Nov. 2010; C.A.S. Souza et al. leg.; CHNUFPI 0040 • 1 \&; Mata da Tijuca; 13 Aug.1977; M. Júlio leg.; UFPB Ar418.

\section{Description}

Male (holotype MNHN 417)

Habitus. See Fig. 18A.

Measurements. TBL 12, CL 6.8, CW 6.1, LL 0.8, LW 1.1, SL 3.8, SW 3.3.

CoLoR. Brown carapace and legs, brownish sternum and coxae (Fig. 18A-B), gray abdomen.

Prosoma. Carapace and ocular arrangement as shown in Fig. 18A. Eye tubercle: 1.4 long; 1.4 wide. Distance AME-ALE 0.6. Eye diameters: AME 0.4, ALE 0.3, PME 0.2, PLE 0.4. Thoracic fovea procurved (Fig. 18A). Labium and sternum without cuspules (Fig. 18B). Basal segment of chelicerae with a prolateral row of 8 teeth, seven larger and one small, and a retrolateral row with 9 small teeth, grouped in basal half (Fig. 19A), with distal rastellum with 10 spines, with same size (Fig. 19B). 

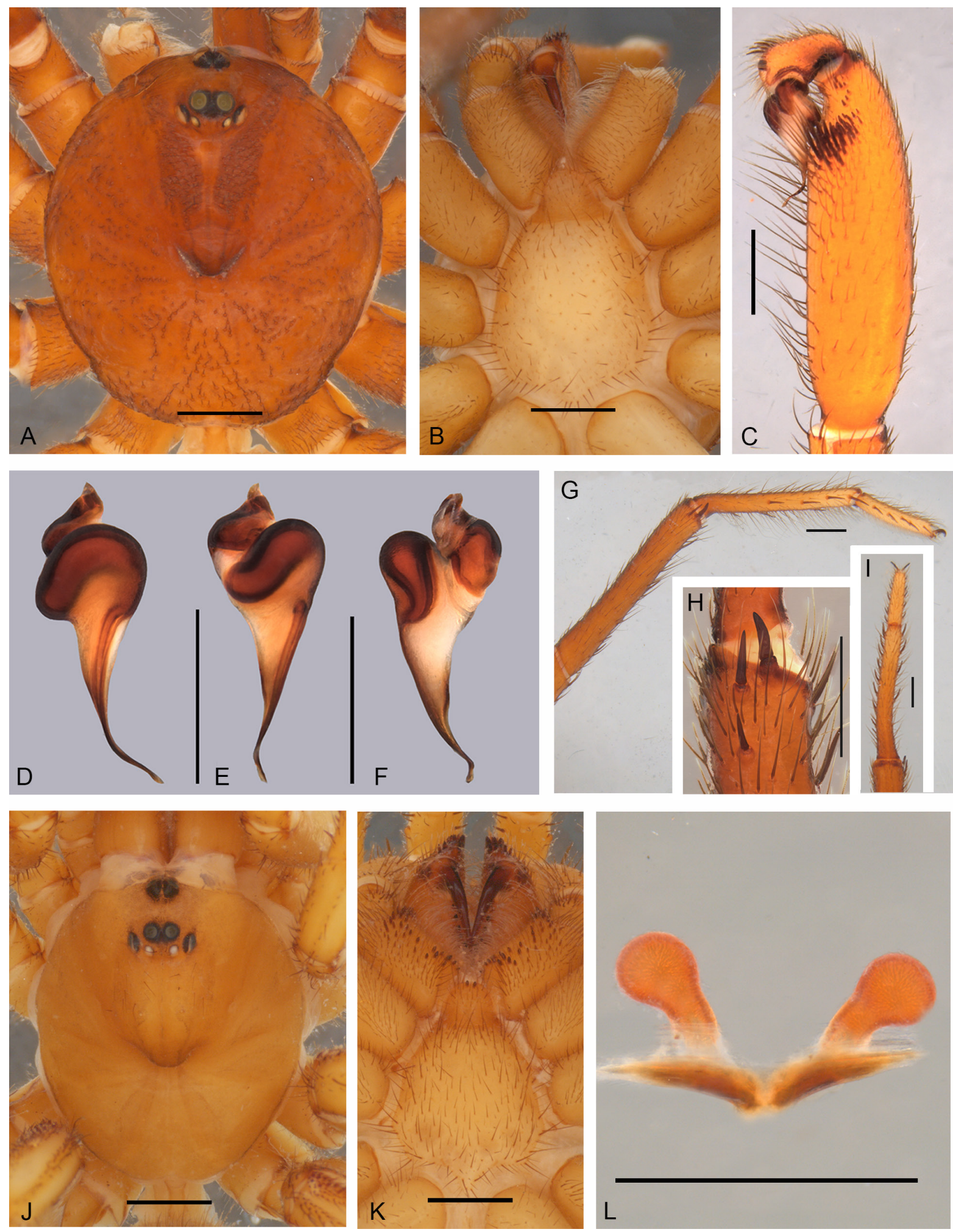

Fig. 18. Idiops germaini Simon, 1892. A-I. $\widehat{\partial}$ (MRNJ). A. Prosoma (dorsal view). B. Prosoma (ventral view). C. Part of palp (retrolateral view). D-F. Palpal bulb. D. Dorsal view. E. Retrolateral view. F. Prolateral view. G. Tibia, metatarsus and tarsus I (prolateral view). H. Tibial apophysis (prolateral view). I. Tibial apophysis and metatarsus I (dorsal view). J-L. q, (CAD 832). J. Prosoma (dorsal view). K. Prosoma (ventral view). L. Genitalia (dorsal view). Scale bars $=1 \mathrm{~mm}$. 
LEGS. Tibia, metatarsus and tarsus I as shown in Fig. 18G.

PALP. Tibia with a shallow retrolateral depression and spines concentrated at basal half of depression (Figs 18C, 19D); elongated embolus with median curvature (Fig. 18E-F) and a small distal embolar tooth close to sperm duct opening (Fig. 19E-F).

PalP AND Leg measurements. Palp $=12$ (4.4, 2, 4.2, 1.4), I $=22.9(7.2,3.4,5.4,4.8,2.1)$, II $=19.9$ (6.4, $2.9,4.4,4.4,1.8), \mathrm{III}=17.2(4.6,2.6,3.3,4.7,2), \mathrm{IV}=24(6.7,3.4,5.6,5.9,2.4)$.

Spination. Palp: Ti r3-4, Ta d0-0-4. Leg I: Fe d1-2-2, Ti p0-0-1, r0-2-2, Mt p0-0-2, r2-2-4, Ta p1-1-1, r2-1-2. Leg II: Fe d1-2-2, Ti v1-1-3ap, Mt p0-1-2, r2-2-2, Ta p0-1-1, r2-2-4. Leg III: Fe d1-1-1, Pa p2-56, r0-0-3, Ti v1-2-2, p1-2-4, r0-1-2, Mt vv2-1-4ap, p3-2-2, r2-1- 1, Ta p2-3-3, r1-2-3. Leg IV: Fe d1-1-2, Pa p13-3-0, Ti v1-1-3, Mt v1-1-1-3, Ta p2- 2-4, r0-0-2.

Female (paratype MNHN 9977)

Habitus. See Fig. 18J.

Measurements. TBL 16.6, CL 9.1, CW 6.7, LL 1.5, LW 1, SL 4.8, SW 4.

CoLoR. Brown carapace and legs, brownish sternum and coxae (Fig. 18J-K), gray abdomen.

Prosoma. Carapace and ocular arrangement as shown in Fig. 18J. Eye tubercle: 2.3 long; 2.8 wide. Distance AME-ALE 0.9. Eye diameters: AME 0.3, ALE 0.5, PME 0.3, PLE 0.5. Thoracic fovea procurved (Fig. 18J). Labium with 2 cuspules (Fig. 18K). Maxilla with 28 cuspules, with larger cuspules concentrated in anterior prolateral and retrolateral extremities (Fig. 18K). Basal segment of chelicerae with a prolateral
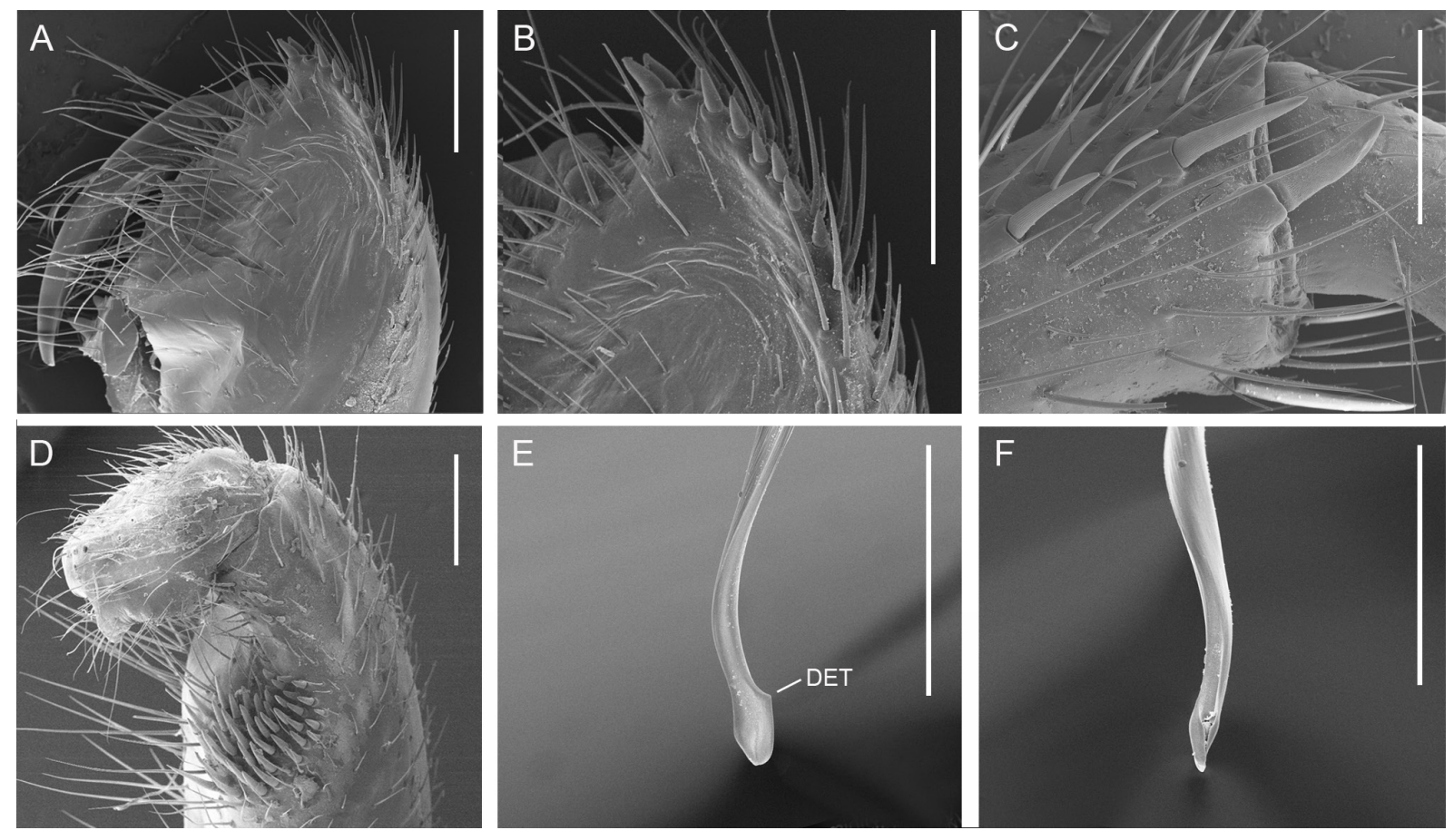

Fig. 19. Scanning electron micrographs of Idiops germaini Simon, 1892, ð (MNRJ). A. Chelicera (retrolateral view). B. Rastellum (retrolateral view). C. Tibial apophysis (dorsal view). D. Part of the palp, showing retrolateral depression. E-F. Detail of the final portion of the embolus. E. Retrolateral view. F. Prolateral view. Abbreviation: $\mathrm{DET}=$ distal embolar tooth. Scale bars $=0.5 \mathrm{~mm}$. 
row of 6 large teeth and a retrolateral row with 13 small teeth, grouped in basal half, rastellum with 14 spines, distal ones longer (Fig. 18K).

Palp and Leg Measurements. Palp $=11.9(4,2.6,2.6,2.7), \mathrm{I}=13.3(4.5,2.9,2.7,2.2,1), \mathrm{II}=11.8(3.8$, $2.7,2.2,2,1), \mathrm{III}=12.9(3.5,2.9,1.9,2.6,2), \mathrm{IV}=17.6(4.8,3.5,3.7,3.5,2.1)$.

Spination. Palp: Pa p0-0-1, Ti p4-9-11, r4-7-13, Ta v0-0-6, p14-9-8, r11-11-10. Leg I: Ti p3-8-11, r3-715, Mt p11-8-6, r11-7-9, Ta v0-0-6, p5-4-2, r6-5-0. Leg II: Ti p1-3-6, r0-1-1, Mt p10-10-10, r5-4-2, Ta v0-0-4, p5-5-4, r3-2-0. Leg III: Pa p3-5-7, r0-0-2, Ti p4-4-4, r0-3-4, Mt v1p-0-0-2, p5-0-3, r5-1-1, Ta v0-2 -7. Leg IV: Pa p30-5-0, Ti v0-1-2, Mt v1-1-1-3ap, Ta v0-2-6.

SPERMATHECAE. Ducts with translucent basal half and sclerotized apical half and spherical receptacula (Fig. 18L).

\section{Distribution}

Brazil. Found in Atlantic Forest phytophysiognomies, with records for the state of Rio de Janeiro (Fig. 3D).

Idiops guri Fonseca-Ferreira, Guadanucci \& Brescovit sp. nov. urn:Isid:zoobank.org:act:A7664A49-43C0-4157-BC48-B98BFB58252B

Figs 3C, 20-21

\section{Diagnosis}

The male of Idiops guri sp. nov. differs from those of other Neotropical species by having the palpal tibia with the retrolateral depression shallow and delimited by spines of decreasing lengths towards the apical portion (Figs 20C, 21D) and by the presence of a hook-shaped projection near the sperm duct opening in retroventral view (Fig. 21E-F). The female differs from the other species by the horseshoe-shaped spermathecae, with ducts curved inward and with sclerotization at the transition between the duct and the receptacle, and oval and weakly sclerotized receptacles (Fig. 20L).

\section{Etymology}

The specific epithet is a tribute to southern Brazil, the region where the species is distributed. The word 'guri', which is of Tupi-Guarani origin, is commonly used in the region to refer to a child or a lad. It also alludes to the tiny size of the specimens.

\section{Type material}

\section{Holotype}

BRAZIL - Rio Grande do Sul • đ̃; São Francisco de Paula, Cento de Pesquisas e Conservação da

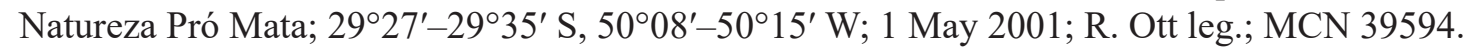

\section{Paratypes}

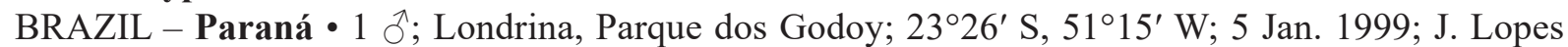
and I.M. Madri leg.; MCTP 11840. - Santa Catarina • 1 đ̇; Chapecó, Floresta Nacional de Chapecó; 2706'10.5" S, 52 46'48.8" W; 10 Mar. 2004; M. Scartezini leg.; IBSP 126721. - Rio Grande do Sul •

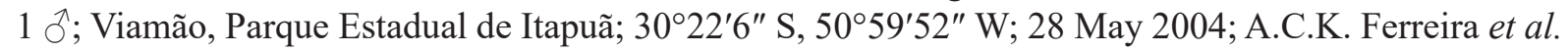
leg.; MCTP 16937.

\section{Other material examined}

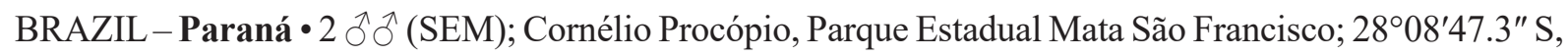
50³4'19.5" W; 2009; J.L. Chavari leg.; IBSP 220523 • 1 đ; Cornélio Procópio, Parque Estadual Mata 
São Francisco; 8 May 2009; N.G. Cipola leg.; IBSP 150273 • 1 đ̊; ; Londrina, Parque dos Godoy; $23^{\circ} 26^{\prime}$ S,

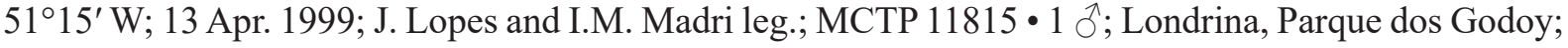

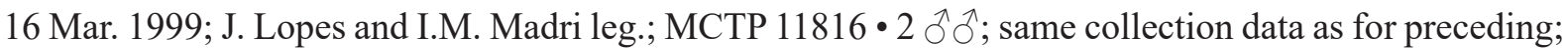
MCTP 11820 • 1 đ; Londrina, Parque dos Godoy; 30 Mar. 1999; J. Lopes and I.M. Madri leg.; MCTP 11817 • 1 ơ; Ponta Grossa, Parque Estadual de Vila Velha; $25^{\circ} 14^{\prime} 17^{\prime \prime}$ S, 500'39" W; 22 Nov. 1986;

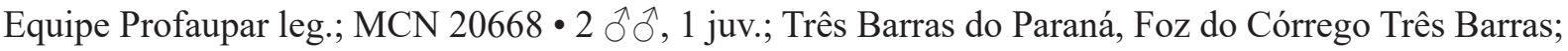

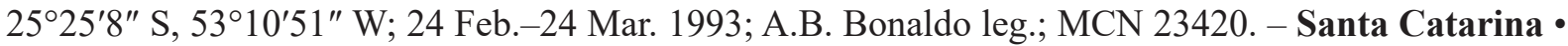

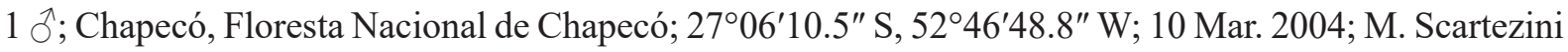

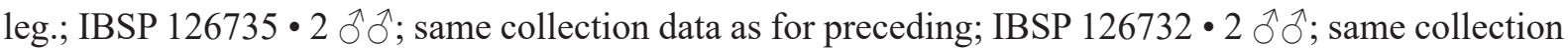
data as for preceding; IBSP $126729 \bullet 8 \hat{\jmath} \bar{\delta}$; same collection data as for preceding; IBSP 126723 to 126724, IBSP 126726 to 126728 , IBSP 126730 to 126731 , IBSP 126733. - Rio Grande do Sul • 2 우; São Francisco de Paula; Potreiro Velho; 29²6'52" S, 50³5'2" W; Feb. 2002; L. Bertocello; MCTP 17532, MCTP 23469 • 1 ; ; São Francisco de Paula, Centro de Pesquisas e Conservação da Natureza Pró-Mata;

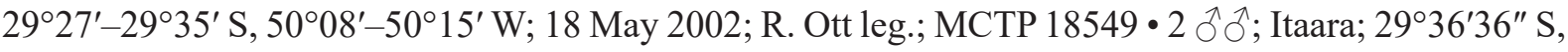
5345'54" W; Apr. 2017; A.A. Lise leg; MCTP 20798 to MCTP 20799 • 1 đ̃, 1 juv.; São Francisco de Paula, Centro de Pesquisas e Conservação da Natureza Pró-Mata; 1 May 2001, R. Ott leg.; MCN 39594a - 2 ふ̂̉, São Francisco de Paula, Centro de Pesquisas e Conservação da Natureza Pró-Mata; 3 Feb.

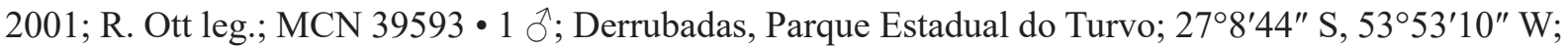

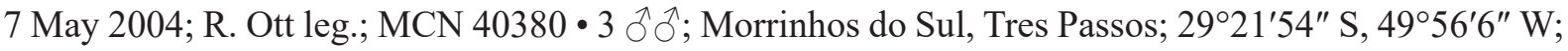
1 Nov. 2006; A. Gonçalves et al. leg.; MCN 52357 to 52358 • 1 đో; Dom Pedro de Alcântara; 29²2'8" S,

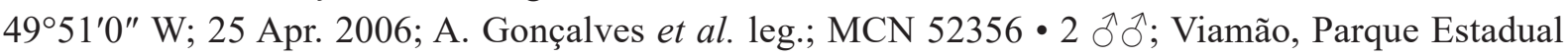
de Itapuã; 30²2'6" S, 5059'52" W; 15 Apr. 2004; A.C.K. Ferreira et al. leg.; MCTP 16939, MCTP

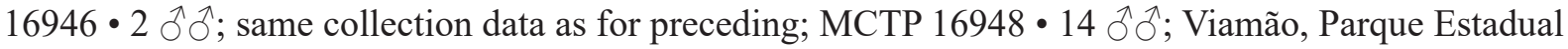

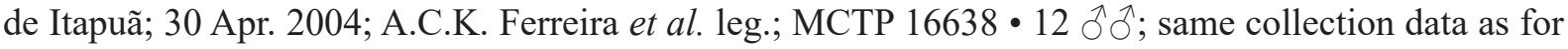
preceding; MCTP 16936 • 1 đ; Viamão, Parque Estadual de Itapuã; 18 Apr.-8 May 2007; R. Moraes leg.;

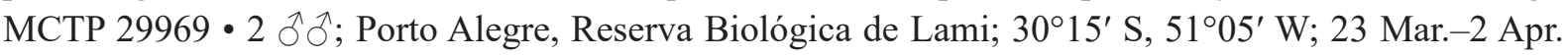
2006; MCN 46338 • 1 § ; Porto Alegre, Reserva Biológica de Lami; 23 Mar.-12 Apr. 2006; R. Moraes

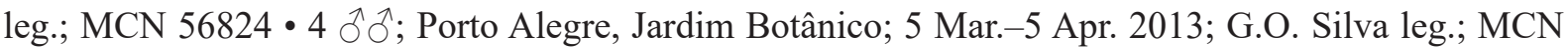

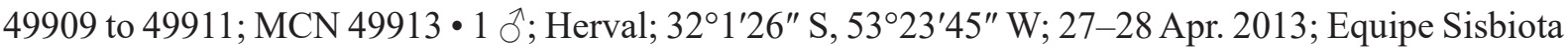
leg.; MCN 48527.

\section{Description}

Male (holotype MCN 39594)

Habitus. See Fig. 20A.

Measurements. TBL 5.3, CL 2.5, CW 2.4, LL 0.4, LW 0.6, SL 1.4, SW 1.3.

CoLOR. Yellowish brown carapace and legs, gray abdomen (Fig. 20A-B).

Prosoma. Carapace and ocular arrangement as shown in Fig. 20A. Eye tubercle: 0.4 long; 0.5 wide. AMEALE distance 0.3. Eye diameters: AME 0.1, ALE 0.2, PME 0.1, PLE 0.12. Thoracic fovea procurved (Fig. 20A). Labium and sternum without cuspules (Fig. 20B). Basal segment of chelicerae with a prolateral row of 5 small teeth equally distributed, rastellum with 11-12 spines, distals largest (Fig. 21A-B).

LEGS. Tibia, metatarsus and tarsus I as shown in Fig. 20G. Tibial apophysis of leg I with short basal branch with small triangular spine and apical branch twice as large as basal branch (Fig. 20G-I), with elongated spine in digitiform shape (Fig. 21C). Pseudoscopula: tarsus I-IV totally covered. 

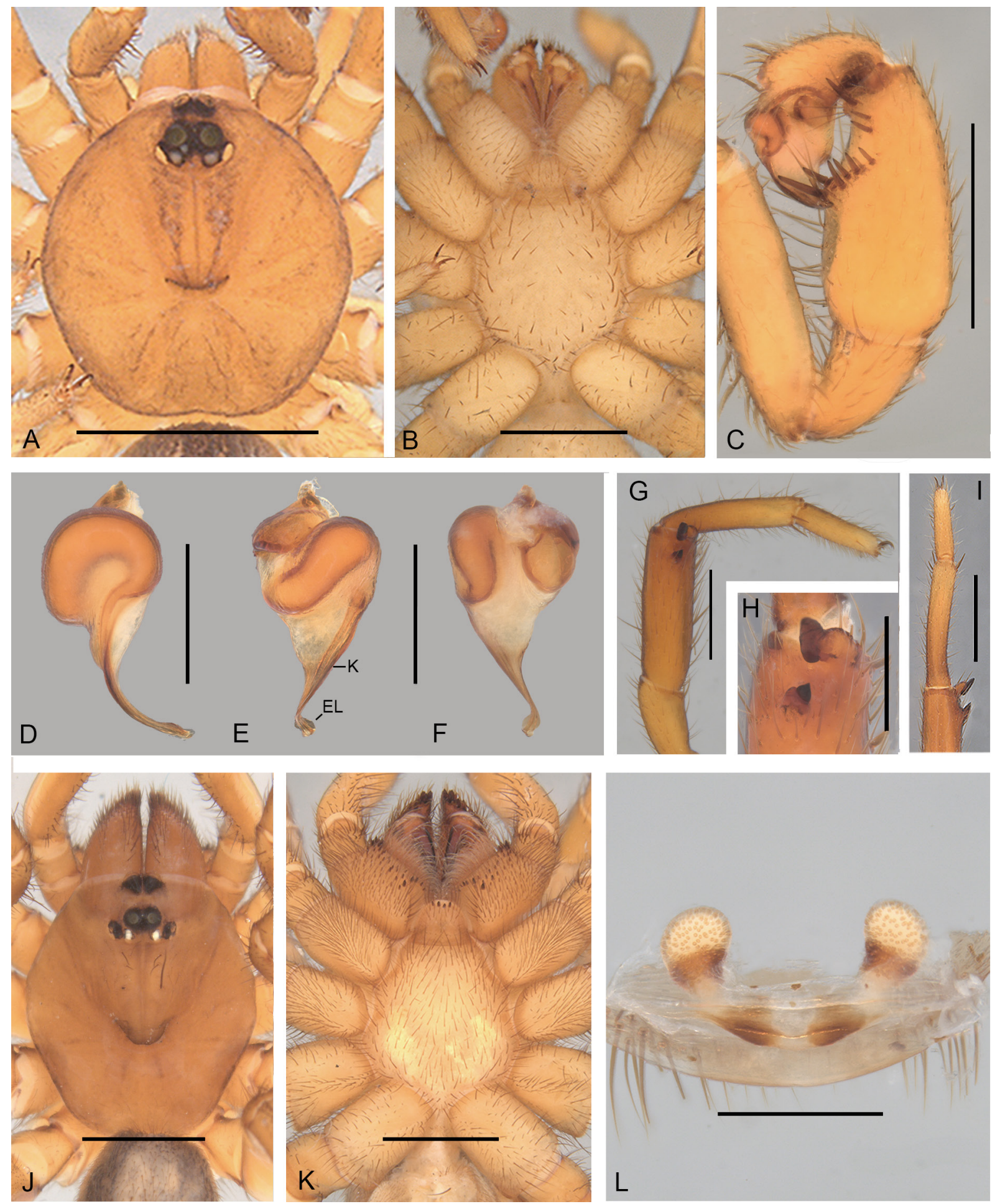

Fig. 20. Idiops guri Fonseca-Ferreira, Guadanucci \& Brescovit sp. nov. A-I. đo, holotype (MCN 39594). A. Prosoma (dorsal view). B. Prosoma (ventral view). C. Part of palp (retrolateral view). D-F. Palpal bulb. D. Dorsal view. E. Retrolateral view. F. Prolateral view. G. Tibia, metatarsus and tarsus I (prolateral view). H. Tibial apophysis (prolateral view). I. Tibial apophysis and metatarsus I (dorsal view). J-L. (MCTP 23469). J. Prosoma (dorsal view). K. Prosoma (ventral view). L. Genitalia (dorsal view). Abbreviations: $\mathrm{EL}=$ embolar lamella; $\mathrm{K}=$ keel. Scale bars: $\mathrm{A}-\mathrm{G}, \mathrm{I}-\mathrm{L}=1 \mathrm{~mm} ; \mathrm{H}=0.5 \mathrm{~mm}$. 
PALP. Tibia with larger spines arranged on basal half of retrolateral depression (Fig. 20C), embolus with keel in retrolateral view, embolus with subapical torsion, followed by lateral lamella close to opening of sperm duct (Figs 20D-F, 21E-F).

PAlP AND Leg Measurements. Palp $=3.8(1.2,0.6,1.4,0.6), \mathrm{I}=7.5(2.2,1.2,1.7,1.4,1), \mathrm{II}=6.8(2.2$, $1,1.4,1.2,1), \mathrm{III}=6.2(1.7,1,1,1.5,1), \mathrm{IV}=8(1.9,1,2,1.9,1.2)$.

Spination. Palp: Ti r13. Leg I: Pa v0-0-1, Ti r0-1-2 Mt v0-0-1, p0-0-1, r1-1-2, Ta p0-0-1 r0-1-0. Leg II: Pa v0-0-3, Ti v1-1-3, p0-0-2, Mt v1-1-3, p0-0-1, Ta p0-0-1. Leg III: Pa p1-2-2, Ti v0-0-2, p0-1-1, r00-1, Mt v0-1-2, p0-1-2, r0-1-2, d0-1-2. Leg IV: Pa 0-0-1, Ti v1-1-2, p0-0-1, r0-0-1, Mt v0-1-2, p0-0-1, r0-0-2, Ta p0-0-1, r0-0-1.

\section{Female (MCTP 23469)}

Habitus. See Fig. 20J.

MeAsurements. TBL 8.4, CL 4.2, CW 3.7, LL 0.7, LW 0.9, SL 2.5, SW 2.5.

COLOR. Brown carapace and legs, gray abdomen (Fig. 20J-K).

Prosoma. Carapace and ocular arrangement as shown in Fig. 20J. Eye tubercle: 0.5 long; 0.9 wide. AME-ALE distance 0.7. Eye diameters: AME 0.2, ALE 0.3, PME 0.2, PLE 0.3. Thoracic fovea procurved (Fig. 20J). Labium with 4 cuspules (Fig. 20K). Maxilla with 29 cuspules, distributed over anterior ventral half, with 8 large cuspules at anterior ventral retrolateral end and 5 large cuspules at anterior ventral prolateral end (Fig. 20K). Basal segment of chelicerae with a prolateral row of 5 large teeth. Rastellum with 11-12 thick spines, distals largest.
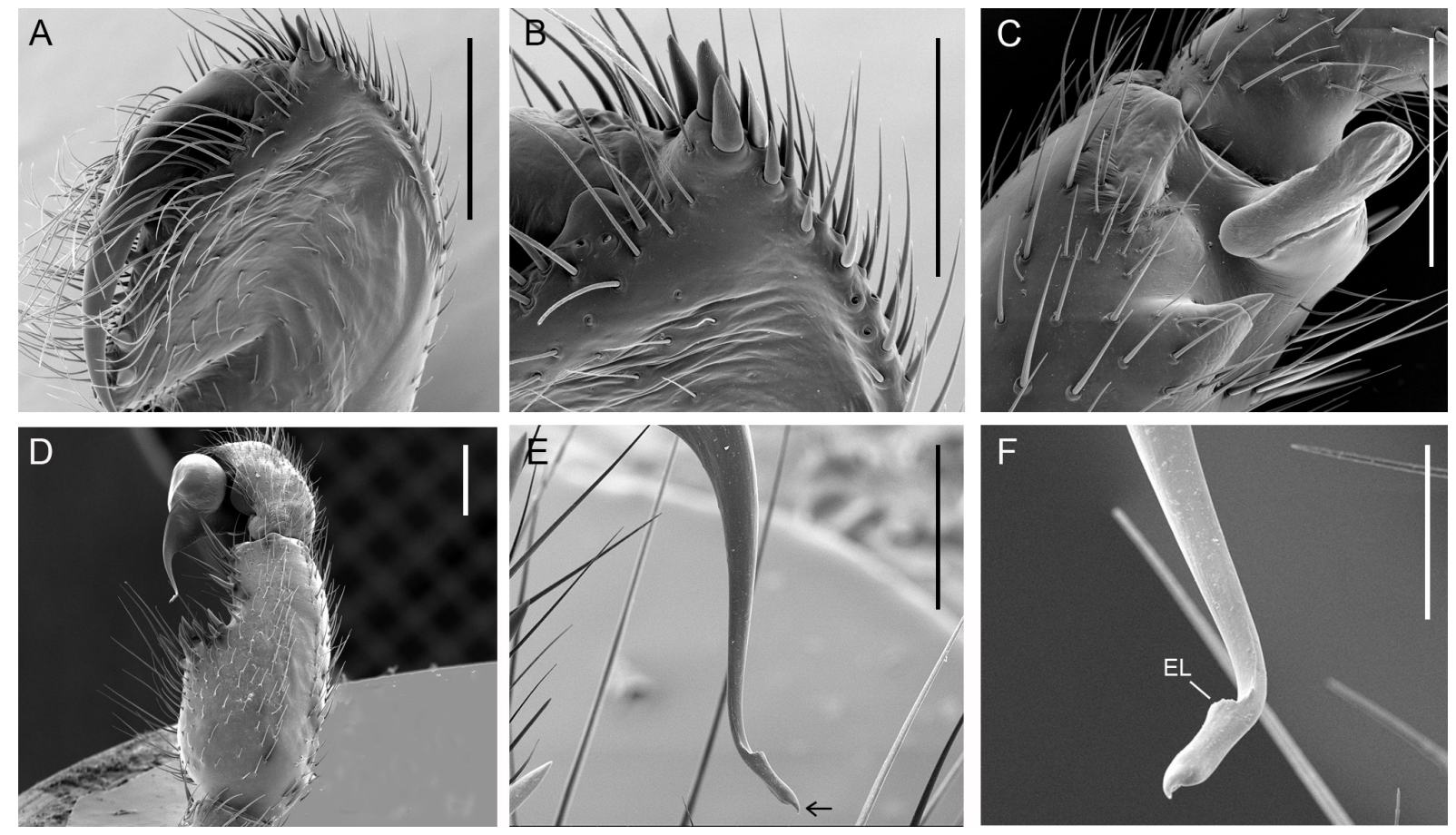

Fig. 21. Scanning electron micrographs of Idiops guri Fonseca-Ferreira, Guadanucci \& Brescovit sp. nov., (IBSP 220523). A. Chelicera (retrolateral view). B. Rastellum (retrolateral view). C. Tibial apophysis (dorsal view). D. Part of the palp, showing retrolateral depression. E-F. Detail of the final portion of the embolus in prolateral view. Abbreviation: $\mathrm{EL}=$ embolar lamella. The arrow indicates the hook-shaped projection near the sperm duct. Scale bars: $A=0.4 \mathrm{~mm} ; \mathrm{B}, \mathrm{E}-\mathrm{F}=0.2 \mathrm{~mm} ; \mathrm{C}=0.3 \mathrm{~mm} ; \mathrm{D}=1 \mathrm{~mm}$. 
Palp and Leg measurements. Palp $=6.9(2.5,1.3,1.5,1.6), \mathrm{I}=7.7(2.5,1.6,1.8,1,0.8), \mathrm{II}=6.6(2.2$, $1.4,1.2,1.1,0.7), \mathrm{III}=7.3(2,1.6,1.2,1.5,1), \mathrm{IV}=9.7(2.6,2,1.8,2,1.3)$.

SPINATIOn. Palp: Ti r5-4-5, p2-4-6, Ta r4-6-6, p7-6-5. Leg I: Mt r3-4-5, p2-3-3, Ta v0-0-3, r5-4-6, p2-4-2. Leg II: Ti r2-3-3, p2-4-2, Mt r6-4-5, p5-5-5, Ta v0-0-3, r3-2-1, p2-4-2. Leg III: Pa p3-5-8, Ti p0-2-3, Mt d2-4-4, v1-2-2, p4-2-1, Ta v0-1-3, p0-2-0. Leg IV: Pa p12-0-0, Ti v1-1-1, Mt v1-2-2, p0-0-1, Ta v0-3-4.

Spermathecae. Duct with translucent median portion and receptacle with evident granules and medial half slightly expanded (Fig. 20L).

\section{Distribution}

Brazil. Found mainly in Atlantic Forest phytophysiognomies, with records for the South region (Paraná, Santa Catarina and Rio Grande do Sul) (Fig. 3C).

\section{Idiops harti (Pocock, 1893)}

Figs 4B, 5C, 22

Pseudidiops harti Pocock, 1893: 407, pl. 19, figs 1-3.

Idiops harti - Raven 1985: 158.

\section{Diagnosis}

The female of Idiops harti differs from that of other species of the genus by having the spermathecae with short ducts (same length as the diameter of the receptacula) and a well-marked division between them, by having the receptacula shaped like a bean (Fig. 22) and by having only an internal row of large teeth on the chelicera (Fig. 5C).

\section{Type material}

\section{Holotype}

TRINIDAD AND TOBAGO • + ; Trinidad; J.H. Hart leg.; BMNH 1893.3.25.1.

\section{Description}

\section{Male}

Unknown.

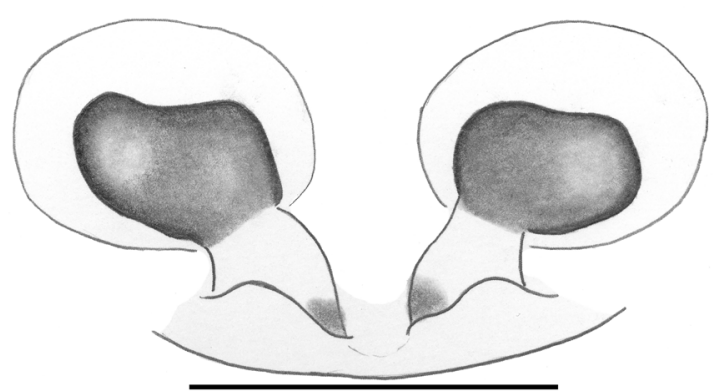

A

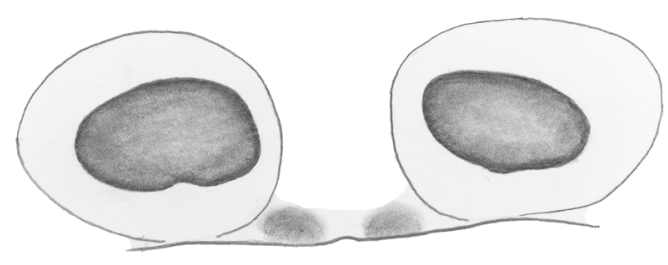

B

Fig. 22. Idiops harti (Pocock, 1893),, , genitalia, holotype (BMNH 1893.3.25.1). A. Dorsal view. B. Frontal view. Scale bar $=1 \mathrm{~mm}$. 
Female (holotype BMNH 1893.3.25.1)

Measurements. TBL 18.8, CL 12.2, CW 9.8, LL 0.8, LW 1.3, SL 5.8, SW 5.6.

CoLor. Carapace and legs light brown. Abdomen brown.

Prosoma. Eye tubercle: 1.7 long; 1.1 wide. AME-ALE distance 0.7. Eye diameters: AME 0.44, ALE 0.4, PME 0.3, PLE 0.4. Thoracic fovea procurved with 7 cuspules. Maxilla with 104 cuspules, distributed through ventral area. Basal segment of chelicerae with a prolateral row of 6 large teeth and one small retrolateral tooth (Fig. 4C).

Palp and leg measurements. Palp $=10.6(3.6,2.4,2.3,2.3), \mathrm{I}=12.8(4.3,2.8,2.9,1.9,0.9), \mathrm{II}=11.5$ $(3.9,2.5,2.3,1.8,1), \mathrm{III}=8.6(2.4,1.9,1.5,1.8,1), \mathrm{IV}=15.8(4.5,2.9,3.5,3.3,1.6)$.

Spination. Palp: Fe p0-0-2, Pa p0-1-1, Ti p6-5-9, r3-6-8, Ta p8-6-9, r9-8-7. Leg I: Ti p3-3-8, r3-5-6, Mt p6-4-4, r5-5-3, Ta p3-1-1, r1-2-1. Leg II: Ti p3-4-6, r1-1-3, Mt p6-5-6, r2-2-4, Ta p2-3-1, r2-2-1. Leg III: Fe d0-0-1, Pa p3-5-9, r0-1-0, Ti v0-0-2, p2-4-7, Mt v0-0-2, p2-1-3, r1-2-1. Leg IV: Pa p0-0-1, Ti v1-1-2, r1-1-1, Mt v1-2-1-3.

SPERmatheCAE. Short ducts, receptacula wider than long (Fig. 22A).

\section{Distribution}

Trinidad and Tobago. Known only from the type locality (Fig. 3B).

Idiops mocambo Fonseca-Ferreira, Guadanucci \& Brescovit sp. nov. urn:Isid:zoobank.org:act:9A7CB56E-31BD-4122-81D0-D514069399D6

Figs 3B, 23A-I

\section{Diagnosis}

The male of Idiops mocambo sp. nov. differs from other Neotropical species by anterior region of the carapace in a pointed shape (Fig. 23A), sternum with distinct black edges (Fig. 23B) and by the apical branch of the tibial apophysis in rectangular shape (Fig. 23G-H).

\section{Etymology}

The specific epithet refers to the location where the holotype specimen was collected.

\section{Type material}

\section{Holotype}

BRAZIL - Pará • O’; Belém, Mocambo; $1^{\circ} 27^{\prime} 21^{\prime \prime}$ S, 48³0'14" W; 4 Nov. 2002; A.B. Bonaldo leg.; MPEG 0110.

\section{Paratype}

BRAZIL - Amazonas • 1 đo'; Manaus, Reserva Florestal Adolpho Ducke; 30'27" S, 5956'22.92" W; 9 Sep. 1991; H. Höfer and T. Gasnier leg.; INPA 4593.

\section{Other material examined}

BRAZIL - Amazonas • 1 ơ; Road Am-010, Km 54; 3³'45" S, 5959'19" W; 6-10 Nov. 1997; J. Vidal et al. leg.; INPA 4594. - Pará • 1 ô’; Barcarena; 1³0'21" S, 48³7’33" W; MPEG 1106. 


\section{Description}

Male (holotype MPEG 0110)

Habitus. See Fig. 23A.

Measurements. TBL 7.9, CL 3.8, CW 3, LL 0.5, LW 0.7, SL 1.9, SW 1.7.

CoLOR. Brown carapace and legs, except for coxae and trochanters, which are light brown (Fig. 23A-B).

Prosoma. Carapace and ocular arrangement as shown in Fig. 23A. Eye tubercle: 0.5 long; 0.9 wide. AME-ALE distance 0.5. Eye diameters: AME 0.2, ALE 0.3, PME 0.2, PLE 0.2. Thoracic fovea procurved (Fig. 23A). Labium and sternum without cuspules (Fig. 23B). Basal segment of chelicerae with a prolateral row of 5 large teeth and 2 small retrolateral teeth, grouped on basal half, rastellum with 16-18 short and thick spines.
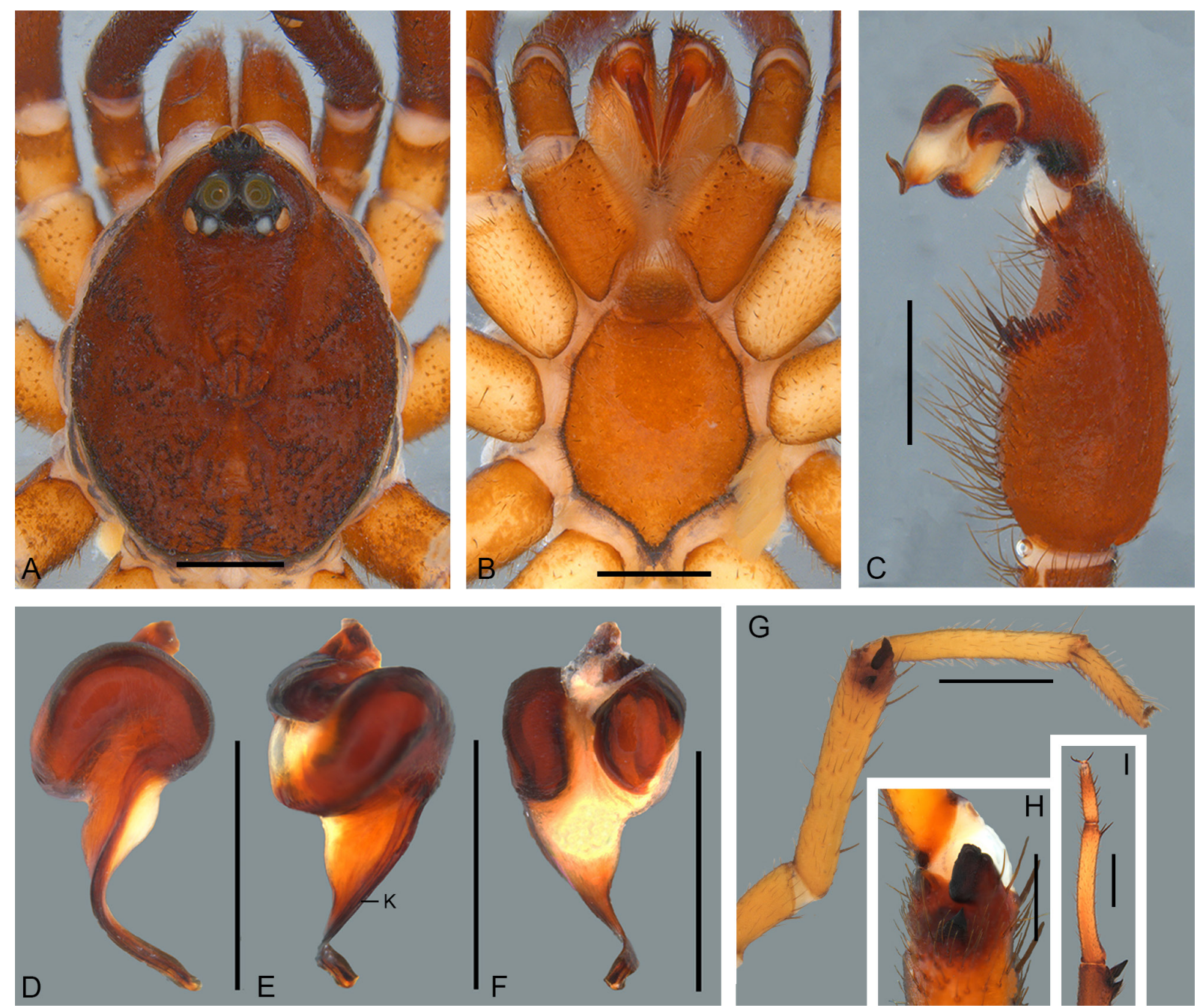

Fig. 23. Idiops mocambo Fonseca-Ferreira, Guadanucci \& Brescovit sp. nov., đ̊, holotype (MPEG 0110). A. Prosoma (dorsal view). B. Prosoma (ventral view). C. Part of palp (retrolateral view). D-F. Palpal bulb. D. Dorsal view. E. Retrolateral view. F. Prolateral view. G. Tibia, metatarsus and tarsus I (prolateral view). H. Tibial apophysis (prolateral view). I. Tibial apophysis and metatarsus I (dorsal view). Abbreviation: $\mathrm{K}=$ keel. Abbreviation: $\mathrm{K}=$ keel. Scale bars: $\mathrm{A}-\mathrm{G}, \mathrm{I}=1 \mathrm{~mm} ; \mathrm{H}=0.5 \mathrm{~mm}$. 
Legs. Tibia, metatarsus and tarsus I as shown in Fig. 23G. Leg I with double tibial apophysis, apical branch twice the size of basal branch (Fig. 23G-I). Pseudoscopula: tarsus I-IV totally covered.

PALP. Tibia with spines distributed along margin of retrolateral depression, with large spines at apical and basal ends (Fig. 23C); embolus with lamelliform apical half, keel in retrolateral view and torsion close to sperm duct opening (Fig. 23D-F).

Palp and leg measurements. Palp $=7.1(2.5,1,2.6,1), \mathrm{I}=11.7(3.4,1.9,2.6,2.6,1.2), \mathrm{II}=10.3(2.8$, $1.5,2.3,2.5,1.2), \mathrm{III}=9.8(2.6,1.4,1.9,2.6,1.3), \mathrm{IV}=12.6(3.2,1.8,3,3.2,1.4)$.

Spination. Palp: Ti r25, Ta 0-0-1. Leg I: Pa v0-0-2, Ti v0-0-2, r1-2-3, Mt p0-0-1, r2-2-2, Ta p1-1-1, r11-1. Leg II: Ti v2-2-3, Mt v3-3-3, r1-1-2, Ta p0-2-0, r1-1-0. Leg III: Ti v0-2-3, Mt v1-2-4, p1-3-4, Ta p0-2-1. Leg IV: Ti v1-1-3, Mt v1-2-4, p1-3-6, Ta p1-1-1.

\section{Distribution}

Brazil. Distributed in phytophysiognomies in the Amazon, with records for the North region (eastern Amazonas and Pará) (Fig. 3B).

Idiops nilopolensis Mello-Leitão, 1923

Figs 3D, 24A-L

Idiops nilopolensis Mello-Leitão, 1923: 47.

Idiops nilopolensis - Bücherl et al. 1971: 121, figs 6-7. — Fukami \& Lucas 2005: 7.

\section{Diagnosis}

Males of Idiops nilopolensis differ from those of other Neotropical species by the strong curvature of the median portion of the embolus in dorsal view (Fig. 24D-F; also present in I. fuscus). Differs from I. fuscus by having the embolus with a thickened basal half and by the small embolar lamella (smaller than in I. fuscus) close to spermatic duct opening (Fig. 24D-F). The female differs from those of other species by having the spermathecae with a translucent duct and without constriction between the duct and the sclerotized receptacles (Fig. 24L).

\section{Type material}

\section{Holotype}

BRAZIL - Rio de Janeiro • +; Rio de Janeiro; Nov. 1923; H. Blanc de Freitas leg.; MNRJ 10. Lost before the 2018 fire (Moreira et al. 2010: 34).

Neotype (here designated)

BRAZIL - Rio de Janeiro • ${ }^{\prime}$; Nova Iguaçu, Parque Municipal de Nova Iguaçu; $22^{\circ} 45^{\prime} 35^{\prime \prime}$ S, $43^{\circ} 27^{\prime} 6^{\prime \prime}$ W; 21 Jul. 2004; R. Baptista leg.; MNRJ.

Remark: The type specimen of Idiops nilopolensis was lost in the MNRJ long before the 2018 fire. In accordance with the criteria of the ICZN Code (ICZN 1999), a neotype is designated here, because the type is lost and the original description is inadequate to stabilise the species. This neotype is based on a specimen collected near the type locality, in the state of Rio de Janeiro, Brazil.

\section{Other material examined}

BRAZIL - Rio de Janeiro • 1 क; same locality as for neotype; 1 Nov. 2004; MNRJ. 


\section{Description}

Male (neotype MNRJ)

Habitus. See Fig. 24A.

MeAsurements. TBL 16.9, CL 7.3, CW 6.4, LL 0.6, LW 1.2, SL 3.9, SW 3.5.

CoLor. Body uniformely light brown (Fig. 24A-B).

Prosoma. Carapace and ocular arrangement as shown in Fig. 24A. Eye tubercle: 0.8 long; 1 wide. AMEALE distance 0.9. Eye diameters: AME 0.4, ALE 0.4, PME 0.2, PLE 0.4. Thoracic fovea procurved (Fig. 24A). Labium and sternum without cuspules (Fig. 24B). Basal segment of chelicerae with a prolateral row of 7 large teeth and 3 small retrolateral teeth, grouped on basal half, rastellum with 16-18 spines, the distal ones longer.

Legs. Tibia, metatarsus and tarsus I as shown in Fig. 20G. Leg I with double tibial apophysis. Apical branch twice the size of basal branch and with a conspicuous spine (Fig. 24H-I). Pseudoscopula: tarsus I-IV totally covered.

PALP. Tibia with retrolateral conspicuous depression and spines concentrated at basal and apical portions (Fig. 24C); embolus with basal torsion and opening of sperm duct in shape of a quill tip (Fig. 24D-E).

Palp and leg measurements. Palp $=11.8(4.3,2.2,3.8,1.5), \mathrm{I}=28.4(8.5,3.8,6.6,6.7,2.8), \mathrm{II}=26.9$ $(7.8,3.4,6,6.3,3.1), \mathrm{III}=23.7(6.1,3.3,4.3,6.4,3.6), \mathrm{IV}=32.6(8.4,3.7,7.9,8.6,4)$.

Spination. Palp: Ti r20, Ta d0-0-1. Leg I: Mt p0-0-2, r2-2-4, Ta p2-2-2, r3-3-4. Leg II: Mt p0-0-2, r0-3-3, Ta p2-3-3, r3-4-5. Leg III: Fe d2-1-1, Pa p2-4-5, Ti d2-2-1, v1-2-2, p4-4-6, Mt d2-2-5, v3-4-5, p3-4-6, r2-2-4, Ta p3-4-2, r3-3-6. Leg IV: Ti v1-2-3, p0-0-1, Mt v2-4-6, p1-2-4, Ta p1-4-6.

\section{Female (MNRJ)}

Habitus. See Fig. 24J.

MeAsurements. TBL 15.7, CL 6.2, CW 5.7, LL 0.9, LW 1.3, SL 3.9, SW 3.4.

CoLor. Brown carapace and legs, brownish sternum and coxae (Fig. 24J-K), gray abdomen.

Prosoma. Carapace and ocular arrangement as shown in Fig. 24J. Eye tubercle: 1.2 long; 0.8 wide. AME-ALE distance 1. Eye diameters: AME 0.2, ALE 0.3, PME 0.2, PLE 0.4. Thoracic fovea procurved (Fig. 24J). Labium with 2 cuspules (Fig. 24K). Maxilla with 48 cuspules, distributed over anterior ventral half, with 6 large cuspules at anterior ventral retrolateral end and 6 large cuspules at anterior ventral prolateral end (Fig. 24K). Basal segment of chelicerae with a prolateral row of 7 large teeth and 5 small retrolateral teeth, grouped on basal half, rastellum with 22 short and thick spines (Fig. 24K).

PalP AND leg measurements. Palp $=11.4(4,2.4,2.4,2.6), \mathrm{I}=12.2(3.7,2.8,2.6,1.7,1.4), \mathrm{II}=11.4$ $(3.6,2.5,2.3,1.6,1.4), \mathrm{III}=13.6(4.1,2.5,2.2,2.1,2.7), \mathrm{IV}=15.7(4.4,3.1,3.1,3.2,1.9)$.

Spination. Palp: Ti p4-10-12, r4-6-8, Ta p6-8-7, r10-13-9. Leg I: Ti v0-2-2, p4-5-6, r1-3-9, Mt v2-2-3, p6-6-9, r4-3-5, Ta v0-0-3, p2-3-3, r4-3-5. Leg II: Fe v0-0-2, Pa v0-0-1, Ti v1-1-2, p4-5-6, r0-0-1, Mt v12-1, p4-6-9, r1-2-1, Ta v0-1-3, p4-4-4, r4-4-2. Leg III: Pa d0-0-2, Ti p2-3-2, r0-0-3, Mt v0-0-1, r3-1-1, 4-4-3, Ta v0-4-3, p0-2-3. Leg IV: Fe d0-0-2, v0-0-3, Ti v1-1-3, Mt v3-4-6, p1-2-3, Ta v3-3-4, p1-2-3.

SPERmatheCAe. Short and narrow sclerotized base, ducts with same width as receptacles (Fig. 24L). 

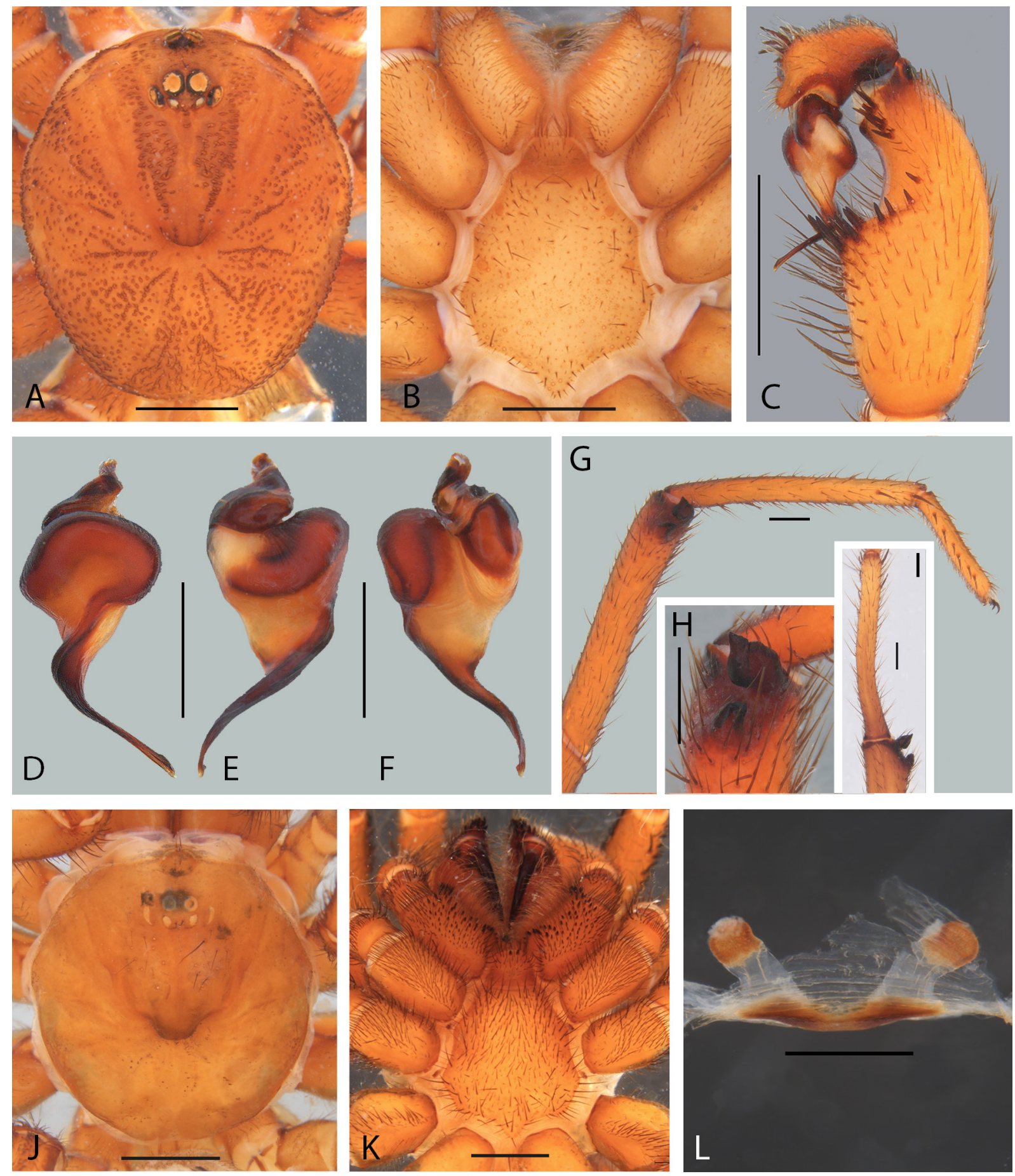

Fig. 24. Idiops nilopolensis Mello-Leitão, 1923. A-I. đ̃, neotype (MRNJ). A. Prosoma (dorsal view). B. Prosoma (ventral view). C. Part of palp (retrolateral view). D-F. Palpal bulb. D. Dorsal view. E. Retrolateral view. F. Prolateral view. G. Tibia, metatarsus and tarsus I (prolateral view). H. Tibial apophysis (prolateral view). I. Tibial apophysis and metatarsus I (dorsal view). J-L. + , (MNRJ). J. Prosoma (dorsal view). K. Prosoma (ventral view). L. Genitalia (dorsal view). Scale bars: A-B, $\mathrm{D}-\mathrm{L}=1 \mathrm{~mm} ; \mathrm{C}=2 \mathrm{~mm}$. 


\section{Distribution}

Brazil. Found in Atlantic Forest phytophysiognomie in the state of Rio de Janeiro (Fig. 3D).

Idiops opifex (Simon, 1889)

Figs 4B, 25

Pseudidiops opifex Simon, 1889: 215, pl. 1 fig. 3.

Idiops opifex - Raven 1985: 139. — Dupérré \& Tapia 2021: 274, figs 16a-b, 17a-b.

\section{Diagnosis}

The female of Idiops opifex differs from that of all other Neotropical species of the genus, except I. rastratus and $I$. duocordibus sp. nov., by presenting cuspules on the entire maxillae ventral face. Differs from I. rastratus by the non-sclerotized spermathecae base (Fig. 25A) and from I. duocordibus sp. nov. by the spherical receptacula (Fig. 25).

\section{Type material}

\section{Holotype}

FRENCH GUIANA • +; Cayenne; MNHN 5340. The holotype is damaged, apparently smashed.

\section{Description}

\section{Male}

Unknown.

Female (holotype MNHN 5340)

Measurements. TBL 11, CL 5.4, CW 5.2, LL 0.9, LW 1.3, SL 2.9, SW 3.3.

CoLor. Carapace and legs brown. Abdomen dark gray.

Prosoma. Eye tubercle: 1.7 long; 1.8 wide. AME-ALE distance 1. Eye diameters: AME 0.3, ALE 0.3, PME 0.2, PLE 0.4. Thoracic fovea procurved. Labium with 23 cuspules. Sternum destroyed. Maxilla with 160 cuspules, distributed through ventral area. Basal segment of chelicerae with a prolateral row of 5 large teeth and 4 small retrolateral basal teeth.
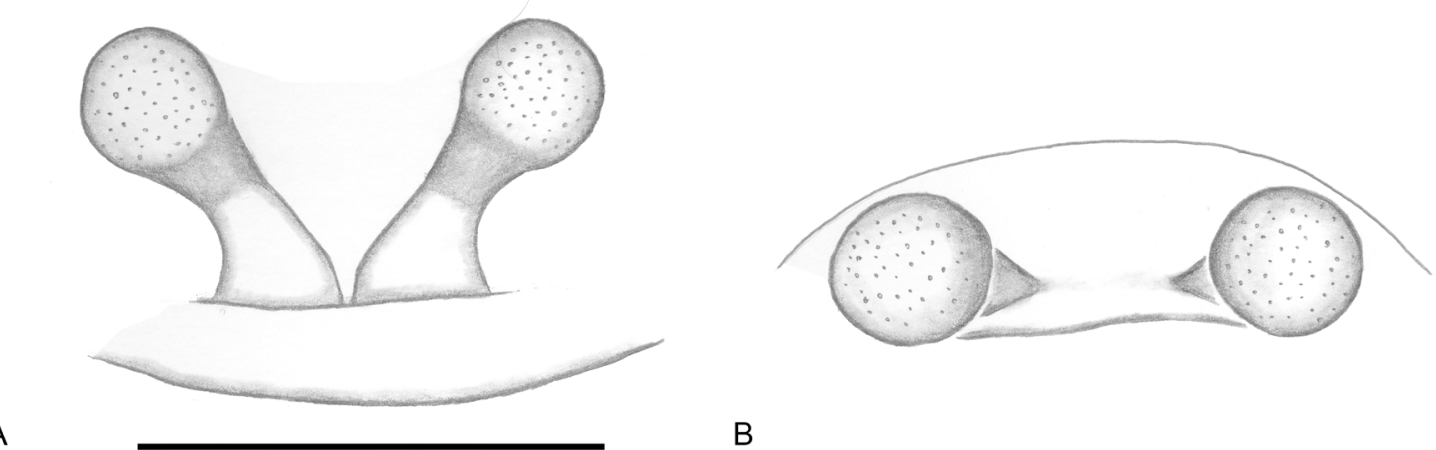

A

B

Fig. 25. Idiops opifex (Simon, 1889),, , genitalia, holotype (MNHN 5340). A. Dorsal view. B. Frontal view. Scale bar $=1 \mathrm{~mm}$. 
Palp and leg measurements. Palp $=10.6(3.6,2.3,2.5,2.2), \mathrm{I}=11.6(3.8,2.7,2.6,1.8,0.7), \mathrm{II}=10$ $(3.4,2.4,2,1.6,0.6), \mathrm{III}=10.7(3.3,2.4,1.8,2,1.2), \mathrm{IV}=14.1(4.4,2.8,3,3.7,1.2)$.

SPINATIOn. Palp: Fe p0-0-3, Pa p0-1-0, Ti p7-9-9, Ta v0-0-2, p11-10-8, r12-9-7. Leg I: Ti p5-7-11, r4-45, Mt p9-7-6, r9-5-6, Ta v0-0-3, p5-2-1, r5-2-0. Leg II: Pa r0-0-1, Ti p3-5-5, r2-3-3, Mt p10-9-5, r5-4-3, Ta p3-3-1, r3-1-0. Leg III: Pa p2-3-9, r0-0-1, Ti v0-0-2, p4-4-10, r3-4-8, Mt v0-0-2, p4-4-6, r2-0-0, Ta v0-0-6. Leg IV: Pa p8-2-0, Mt v1-1-1-3, Ta v0-0-6.

SPERMATHECAE. Duct length similar to diameter of spherical receptacles, close to each other, with basal half thicker than apical half and with sclerotization at transition between duct and receptacle (Fig. 25).

\section{Distribution}

French Guyana. Know only from type locality (Fig. 4B).

\section{Idiops petiti (Guérin, 1838)}

Figs 3B, 26

Acanthodon petitii Guérin, 1838: 163, pl. 47 figs 1-8.

Acanthodon santaremia O. Pickard-Cambridge, 1896: 733, pl. 34 fig. 13. Syn. Nov.

Idiops crulsi Mello-Leitão, 1930: 55, fig. 2. Synonymized by Bücherl et al. 1971: 128.

Idiops petiti - O. Pickard-Cambridge 1870: 107; 1896: 732, pl. 34 figs 9-12. — Mello-Leitão 1923: 48.

— Bücherl et al. 1971: 121, fig. 5.

Idiops santaremius - Petrunkevitch 1911: 73.

\section{Diagnosis}

The male of Idiops petiti differs from that of other Neotropical species, except I. carajas, by having the palpal tibia with spines concentrated in the basal half of the retrolateral depression (Fig. 26C), the tibial apophysis with a narrow apical branch and rectangular in shape (Fig. 26G-H) and by the presence of a lateral lamella that extends along the median portion of the embolus (Fig. 26E-F). Differs from I. carajas by having the subapical portion of the embolus thin and straight (Fig. 26E-F) and the metatarsus of the leg I slightly curved and with a small prolateral projection on the apical half (Figs 26I). Females are distinguished from congeners, except I. carajas, by having the spermathecae with a large sclerotized trapezoidal base and V-shaped ducts (Fig. 26L). Differs from I. carajas by its large oval-shaped receptacula (Fig. 26L).

\section{Type material}

Holotype of Acanthodon petitii Guérin, 1838

BRAZIL - Pará • 9 ; Santarém; BMNH 1890.7.1.320.

Holotype of Acanthodon santaremia O. Pickard-Cambridge, 1896

BRAZIL - Pará • juv.; Santarém; Mar. 1896; O. P.-Cambridge leg.; BMNH 1896.12.13.66.

\section{Holotype of Idiops crulsi Mello-Leitão, 1930}

BRAZIL - Pará • + ; Oriximná, Rio Cuminá; G. Cruls leg.; MNRJ 0007. Lost in the fire of 2018.

The type specimen of Idiops petiti was originally deposited in a dry collection and subsequently rehydrated and stored in alcohol. Although clearly an adult female, the specimen has lost many legs and the spermathecae, because the abdomen was stuffed with cotton. This prevented a more detailed description. The type specimen of I. santaremius was examined and recognized as a juvenile of I. petiti. 


\section{Other material examined}

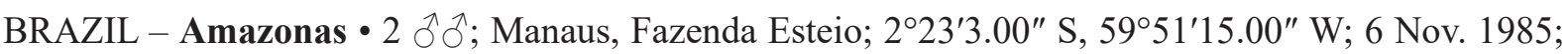
B.C. Klein leg.; INPA 4590 to $4591 \bullet 10^{\top}$; Benjamin Constant; $4^{\circ} 22^{\prime} 58^{\prime \prime}$ S, $70^{\circ} 1^{\prime} 51^{\prime \prime}$ W; 2014; P.S. Pompeu

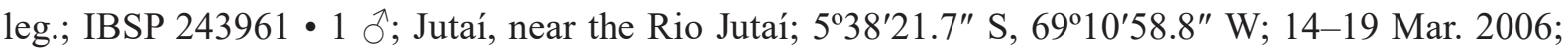

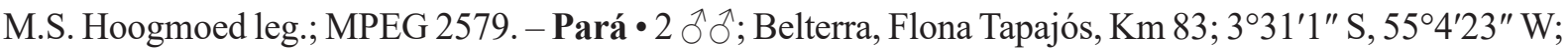
2010; J.L. Freitas leg.; IBSP 218829, IBSP 218868 • 1 ô;; Almeirim, Jari; 1¹'33.122" S, 52³4'2.785" W; MPEG 7588 • 1 §`; Senador José Porfirio; Trilha do Censo, Margem Direita, Rio Xingu; $2^{\circ} 35^{\prime} 27^{\prime \prime}$ S,

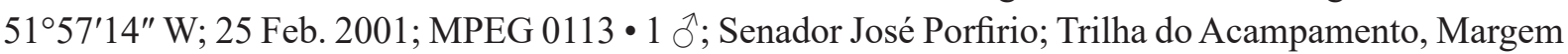
Direita, Rio Xingu; 25 Feb. 2001; MPEG 0116 • 1 đ; Jacareacanga, UHN de São Manoel; 9¹3'32" S, 5659'56" W; 16 Feb. 2009; W.U. Prado leg.; UFMG 3205 • 1 đo; Jacareacanga; 21 Feb. 2009; W.U. Prado leg.; UFMG 3206. - Rondônia • 1 क; Monte Negro, LC25, Km 10; 10¹4'49" S, 6324'16.8” W; 19 Dec. 2013; P.H. Martins leg.; UFMG 24031.

\section{Description}

Male (MPEG 0116)

Habitus. See Fig. 26A.

Measurements. TBL 17.2, CL 7.4, CW 6.7, LL 0.9, LW 1.4, SL 4, SW 3.6.

COLOR. Carapace and legs reddish brown, with yellowish coxae, brownish sternum; abdomen grey (Fig. 26A-B)

Prosoma. Carapace and ocular arrangement as shown in Fig. 26A. Eye tubercle: 0.7 long; 1.3 wide. AME-ALE distance 0.9. Eye diameters: AME 0.4, ALE 0.4, PME 0.2, PLE 0.4. Thoracic fovea procurved (Fig. 26A). Labium and sternum without cuspules (Fig. 26B). Basal segment of chelicerae with a prolateral row of 8 large teeth and 4 small retrolateral teeth, grouped in basal half, rastellum presenting 12-13 spines of same size.

LeGs. Tibia, metatarsus and tarsus I as shown in Fig. 26G. Leg I with double tibial apophysis. Apical branch twice the size of basal branch and with a conspicuous spine (Fig. 26H-I). Pseudoscopula: tarsus I-IV totally covered.

PALP. Elongated palpal bulb with slightly curved embolus and without torsion of subapical portion (Fig. 26D-F).

Palp and leg measurements. Palp $=12(3.8,3.3,3.21,1.7), \mathrm{I}=22(6.8,3.5,5.1,4.8,1.8), \mathrm{II}=18.9$ (6, $3,4.4,3.8,1.7), \mathrm{III}=17(4.7,2.9,3,4.3,2.1), \mathrm{IV}=24.2(6.9,3.6,5.9,5.1,2.7)$.

Spination. Palp: Ti r19, Ta d0-0-4. Leg I: Fe d1-2-1, Pa v0-1-5, Ti v10-15-16, p0-1-0, Mt v6-8-14, p0-0-5, r0-0-4, Ta p3-4-4, r5-6-6. Leg II: Fe d1-2-1, Pa p0-0-2, Ti v5-7-12, p2-2-9, r1-2-4, Mt v6-6-7, p3-5-10, r3-3-4, Ta p4-4-4, r6-6-7. Leg III: Fe d1-2-1, Ti p2-3-6, Mt r6-3-8, Ta p5-8-8, r2-4-7. Leg IV: Fe d1-2-1, Ti v1-2-3, r0-1-2, Mt v1-3-3, p1-2-5, r0-3-5, Ta p5-5-8, r5-5-8.

Female (UFMG 24031)

Habitus. See Fig. 26J.

MeAsurements. TBL 13, CL 5.8, CW 4.9, LL 0.8, LW 2, SL 3.5, SW 3.5.

CoLOR. Carapace and legs brown, light brown sternum (Fig. 22J-K). Abdomen grey. 
Prosoma. Carapace and ocular arrangement as shown in Fig. 26J. Eye tubercle: 0.6 long; 1 wide. AMEALE distance 0.8. Eye diameters: AME 0.2, ALE 0.3, PME 0.2, PLE 0.4. Thoracic fovea procurved (Fig. J). Labium with 3 cuspules (Fig. 26K). Maxilla with 30 cuspules, distributed throughout anterior ventral half (Fig. 26K). Basal segment of chelicerae with a prolateral row of 7 large teeth and 4 small
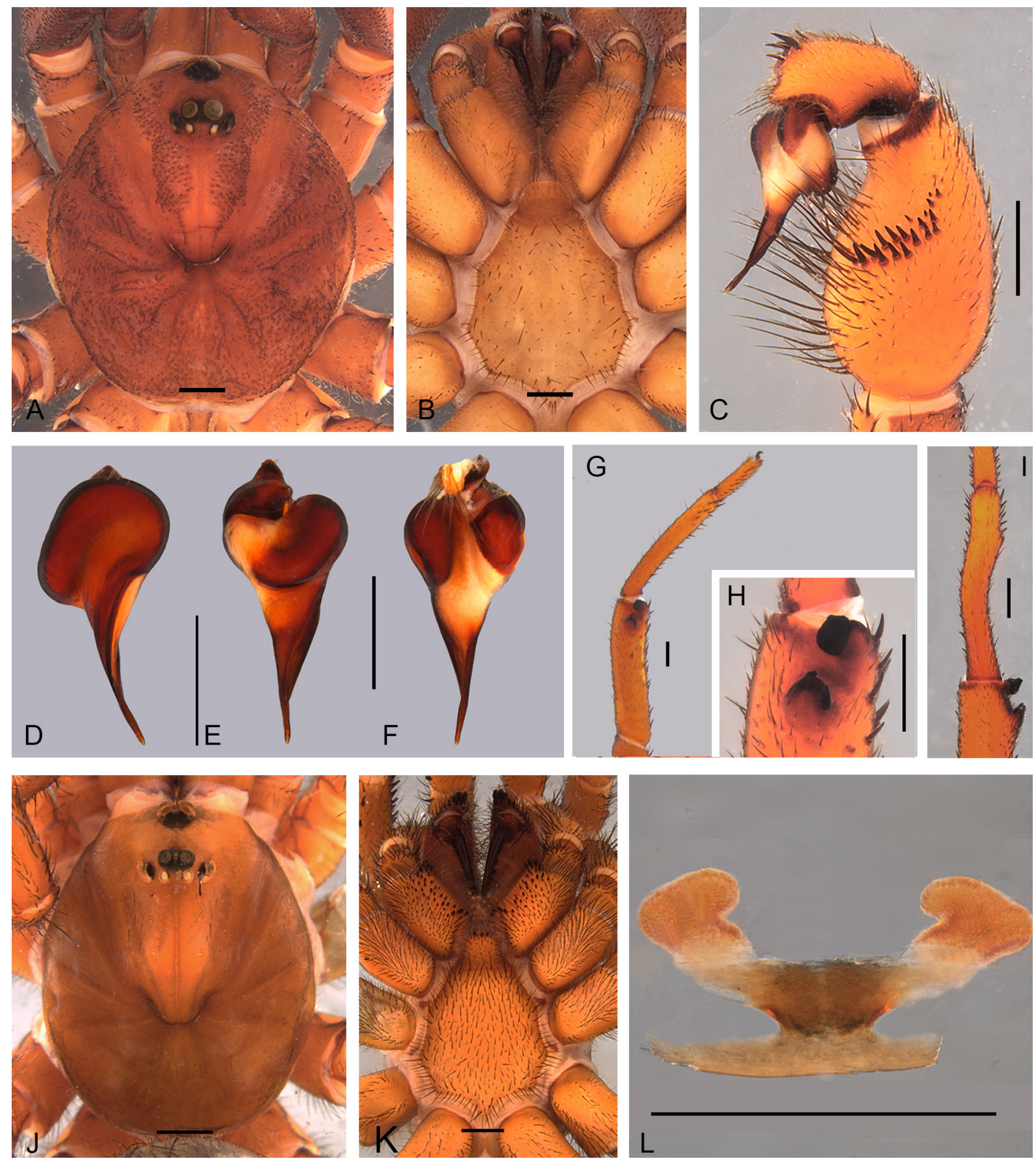

Fig. 26. Idiops petiti (Guérin, 1838). A-I. §̊ (MPEG 0116). A. Prosoma (dorsal view). B. Prosoma (ventral view). C. Part of palp (retrolateral view). D-F. Palpal bulb. D. Dorsal view. E. Retrolateral view. F. Prolateral view. G. Tibia, metatarsus and tarsus I (prolateral view). H. Tibial apophysis (prolateral view). I. Tibial apophysis and metatarsus I (dorsal view). J-L. \& (UFMG 24031). J. Prosoma (dorsal view). K. Prosoma (ventral view). L. Genitalia (dorsal view). Scale bars $=1 \mathrm{~mm}$. 
retrolateral teeth, grouped in basal half. Rastellum well developed, presenting 14-16 robust spines (Fig. 26K).

PalP and Leg measurements. Palp $=9.6(3,2,2.2,2.4), \mathrm{I}=10.2(2.8,2.2,2.3,1.7,1.2), \mathrm{II}=10.2(3.3$, $2.2,2,1.3,1.4), \mathrm{III}=11.3(3,2.3,2,2.3,1.7), \mathrm{IV}=15.2(4,3,3.2,2.9,2.1)$.

SpinAtion. Palp: Ti p5-6-9, r6-8-12, Ta p6-7-8, r8-8-9. Leg I: Pa v0-0-2, Ti v1-2-1, p4-6-9, r4-6-11, Mt v2-1-1, p8-6-8, r10-3-4, Ta v0-0-1, p3-4-4, r4-3-2. Leg II: Ti v1-1-1, p3-7-7, r0-1-2, Mt v1-1-1, p7-5-4, r1-2-2, Ta v0-1-1, p4-4-3, r2-2-1. Leg III: Fe d0-0-1, Pa d0-0-1, p4-5-8, r0-0-1, Ti v0-1-2, p3-4-5, r0-14, Mt v2-1-2, p2-3-2, r2-2-1, Ta v1-2-3, p0-0-3. Leg IV: Fe d0-0-1, Pa p12-4-2, Ti v1-1-2, Mt v2-3-5, p0-1-2, Ta v1-2-3, p0-3-6.

SPERMATHECAE. Ducts with weakly sclerotized basal half and thickened and sclerotized apical half; asymmetric receptacula, with expansion of medial half (Fig. 26L).

\section{Distribution}

Brazil. Widely distributed in the Amazon region, with records for the North region (Amazonas, Pará and Rondônia) (Fig. 3B).

Idiops pirassununguensis Fukami \& Lucas, 2005

Figs 2E-F, 4C, 6G, 27-28

Idiops pirassununguensis Fukami \& Lucas, 2005: 2, figs 1-5.

\section{Diagnosis}

Males of Idiops pirassununguensis differ from those of other Neotropical species by having the tibial apophysis with a robust apical branch and a prominent spine in prolateral view (Figs 27H-I, 28C), metatarsus I long with a prolateral projection on the apical half (Fig. 27I) and the embolus with a prominent lamella that expands along the apical half, with a constriction near the sperm duct opening (Figs 27D-F, 28E-F). Females differ by having the spermathecae with long vertical ducts, and strong sclerotization on the transition between duct and receptacula, with the same diameter as ducts (Fig. 27L).

\section{Type material}

\section{Holotype}

BRAZIL - São Paulo• ○’ ; Pirassununga; Nov. 1997; P. Valdujo leg.; IBSP 9565.

\section{Other material examined}

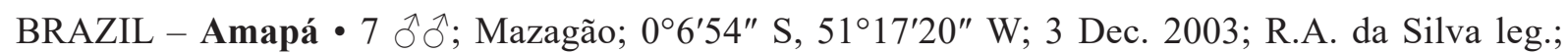

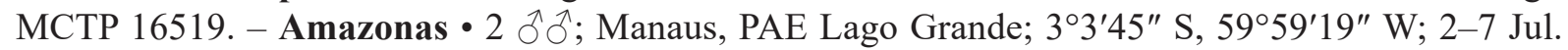
2007; Projeto Geoma 2 leg.; INPA 6914, INPA 6916 • 1 q; Manaus, PAE Lago Grande; 5 May 2007; A.L. Tourinho and R. Saturnino leg.; INPA 6915. - Pará • 1 ố; Parauapebas; 64'4" S, 49 54'7" W; Sep.

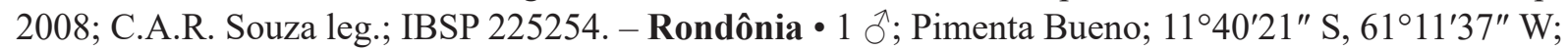

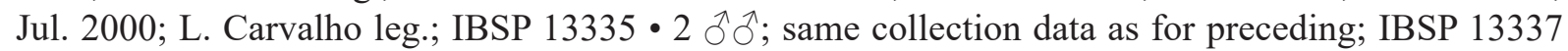

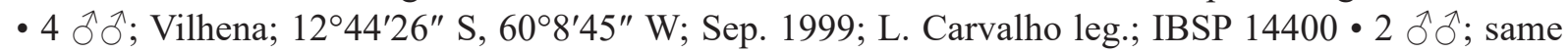

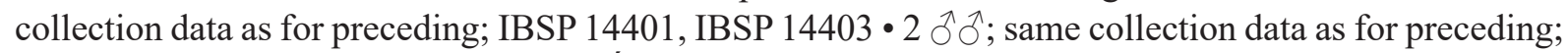
IBSP 14402.- Maranhão • 1 Oें $^{\wedge}$ Caxias, Área de Proteção Ambiental Municipal do Inhamum; 04 53'30" S,

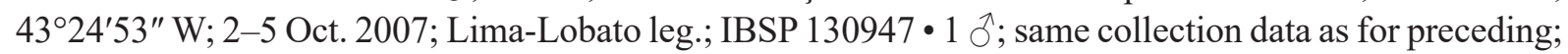

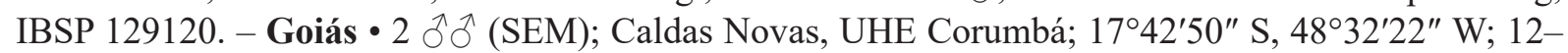
23 Aug. 1996; M.T.I. Rodrigues et al. leg.; MZSP 15651. - Piauí • 5 ổ đ̂; Alvorada do Gurguéia, Fazenda 
Escola UFPI; 8²2'11.5" S, 4351'30.2" W; 4-12 Sep. 2018; D.B.S. Barbosa et al. leg.; CHNUFPI 2715

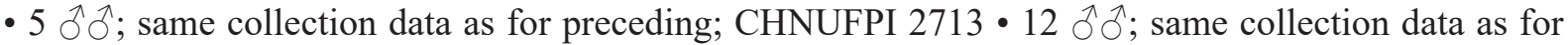

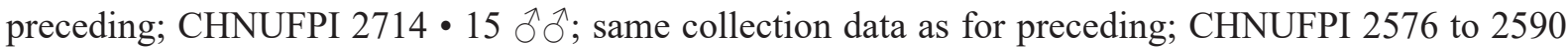

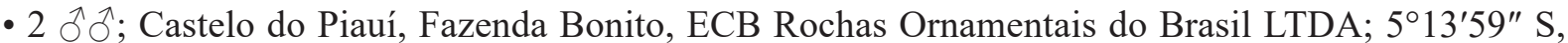

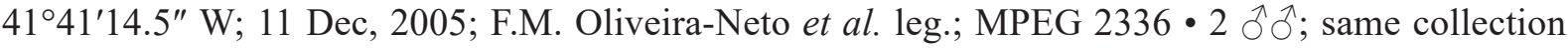
data as for preceding; MPEG 2339, MPEG 2401 • 1 त; Castelo do Piauí, Fazenda Bonito, ECB Rochas

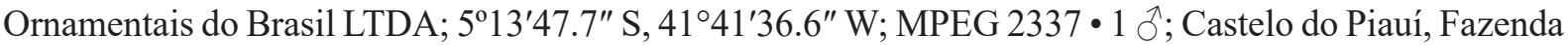

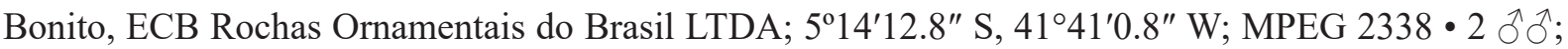
Castelo do Piaú, Fazenda Bonito, ECB Rochas Ornamentais do Brasil LTDA; $5^{\circ} 19^{\prime} 19^{\prime \prime}$ S, 41 ${ }^{\circ} 33^{\prime} 10^{\prime \prime}$; CHNUFPI CASTa0226 1 \% ; same collection data as for preceding; CHNUFPI CASTa0225 • 1 ; ; Castelo do Piauí, Fazenda Bonito, ECB Rochas Ornamentais do Brasil LTDA; 5¹3'46.7" S, 41²41'29.9" W; 25 Oct. 2005; F.M. Oliveira-Neto et al. leg.; CHNUFPI $4024 \bullet 2 \delta^{\lambda} \sigma^{\lambda}$; Castelo do Piauí, Fazenda Bonito, ECB Rochas Ornamentais do Brasil LTDA; $5^{\circ} 13^{\prime} 59^{\prime \prime} \mathrm{S}, 41^{\circ} 41^{\prime} 14.5^{\prime \prime} \mathrm{W} ; 8$ May 2005; F.M. Oliveira-Neto

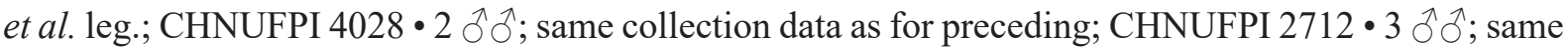
collection data as for preceding; CHNUFPI 4025 to $4027 \bullet 1$ \%; Guaribas, Parque Nacional da Serra das

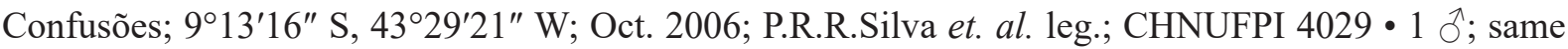

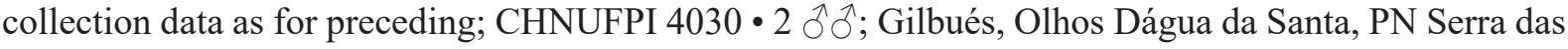

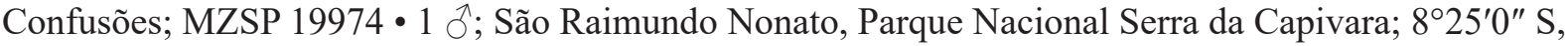
$42^{\circ} 20^{\prime} 0^{\prime \prime}$ W; 27 Oct. -9 Nov. 2012; R. Recoder and M. Teixeira Jr. leg.; IBSP 167608 • 1 ठ̊; Uruçuí, Vale

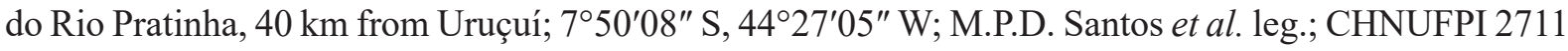

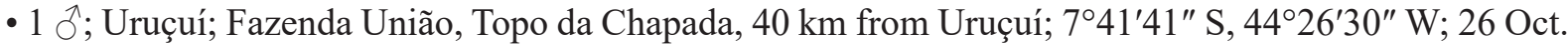
2007; F.M. Oliveira-Neto leg.; CHNUFPI 2710. - Paraíba • 5 đ̊ ô; São José dos Cordeiros, RPPN Almas; $7^{\circ} 28^{\prime} 45^{\prime \prime}$ S, 3654'18" W; 2008-2009; A. Vasconcelos leg.; IBSP 228197, IBSP 228200 to 228201,

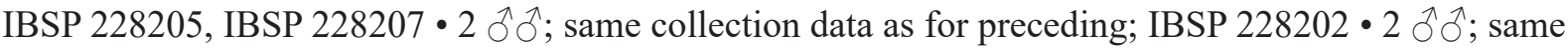

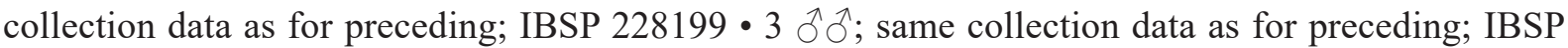
228193. - Sergipe • 1 đ̃; Canindé do São Francisco, Fazenda São José-Olhos D'água, UHE de Xingó;

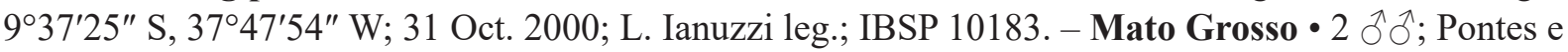
Lacerda; $15^{\circ} 19^{\prime} 10.38^{\prime \prime}$ S, 59¹7'33.70" W; 5-10 Oct. 2013; R.A.K. Ribeiro leg.; UFMT • 2 ô ô;; Vila Bela da Santíssima Trindade, Córrego Areias; 1450'29.9" S, 69³9'01.2" W; 2-4 Nov. 2013; R.A.K. Ribeiro leg.; UFMT • $1 \mathrm{o}^{\Uparrow}$; Lucas do Rio Verde, PCH Canoa Quebrada; $12^{\circ} 47^{\prime} \mathrm{S}, 56^{\circ} 00^{\prime} \mathrm{W}$; V. Azaias leg.; UFMT. - Minas Gerais - $1{ }^{\lambda}$; Santana do Riacho, Parque Nacional Serra do Cipó; $19^{\circ} 22^{\prime} 1^{\prime \prime}$ S, 4332'17" W;

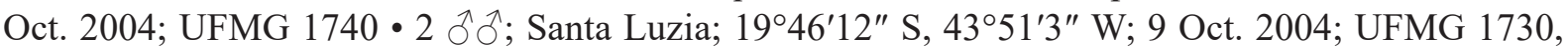

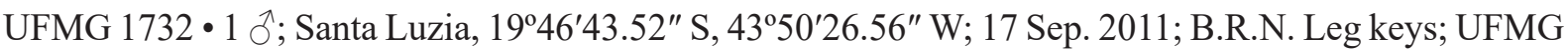

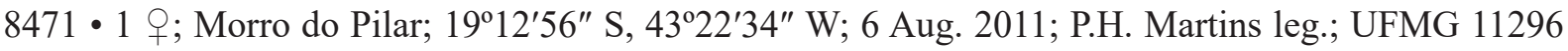

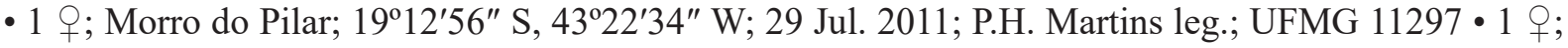

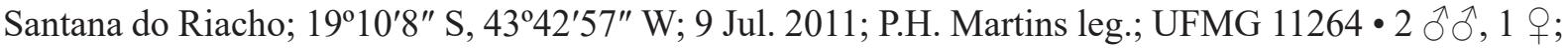
Santana do Riacho, Parque Nacional Serra do Cipó; 19²1'06.8" S, 43³6'38.8" W; 17-21 Oct. 2018; R.F. Ferreira, A. Galleti, P.H. Martins and V. Ghirotto leg.; CAD 833 • 1 क ; Diamantina, Campus JK;

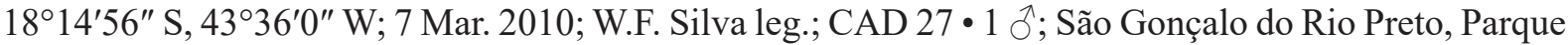
Estadual do Rio Preto; $18^{\circ} 10^{\prime} 29.97^{\prime \prime}$ S, 4320'41.25" W; 20-25 Oct. 2010; G. Monteiro, F. Sá, W.F. Silva and J.P.L. Guadanucci leg.; CAD 275 • 1 क; São Gonçalo do Rio Preto, Parque Estadual do Rio Preto; 14 Jan. 2010; J.P.L. Guadanucci, W.F. Silva, D. Moura, R. F. Ferreira and D. Weinmann leg.; CAD 14

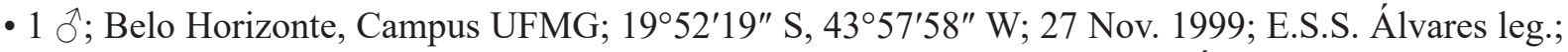
UFMG 606 2 ठð; Belo Horizonte, Campus UFMG; 10-25 Sep. 2000; E.S.S. Álvares leg.; UFMG 607

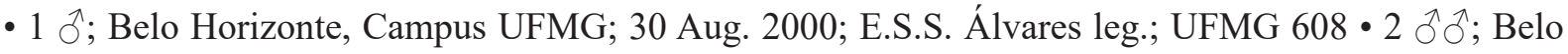
Horizonte, Campus UFMG; 26 Sep. 2009; I.L.F. Magalhães leg.; UFMG 3211 • 1 ơ; Belo Horizonte, Reserva Biológica UFMG; IBSP 14488 - 1 đ; Belo Horizonte, Campus UFMG, Centro Pedagógico UFMG; Jul. 2002; C. Torres leg.; IBSP 13659. - São Paulo • 1 '̊; Araraquara; $21^{\circ} 47^{\prime} 38^{\prime \prime}$ S, 48 $18^{\circ} 10^{\prime} 33^{\prime \prime}$ W; 30 Sep. 1988; IBSP 9562 • 1 ơ; Ribeirão Preto; $21^{\circ} 10^{\prime} 40^{\prime \prime}$ S, 4748’36" W; 24 Nov. 2004; IBSP 12337 • 


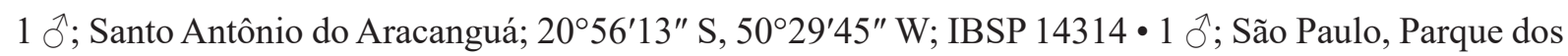
Príncipes; $23^{\circ} 25^{\prime} \mathrm{S}, 46^{\circ} 37^{\prime} \mathrm{W}$; IBSP $13324 \cdot 1{ }^{\top}$; Rio Pequeno; $23^{\circ} 33^{\prime} \mathrm{S}, 46^{\circ} 43^{\prime} \mathrm{W}$; IBSP $3346 \bullet 1$ ○’;

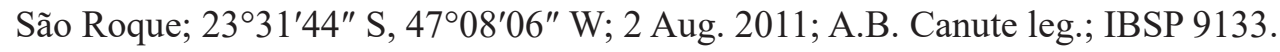
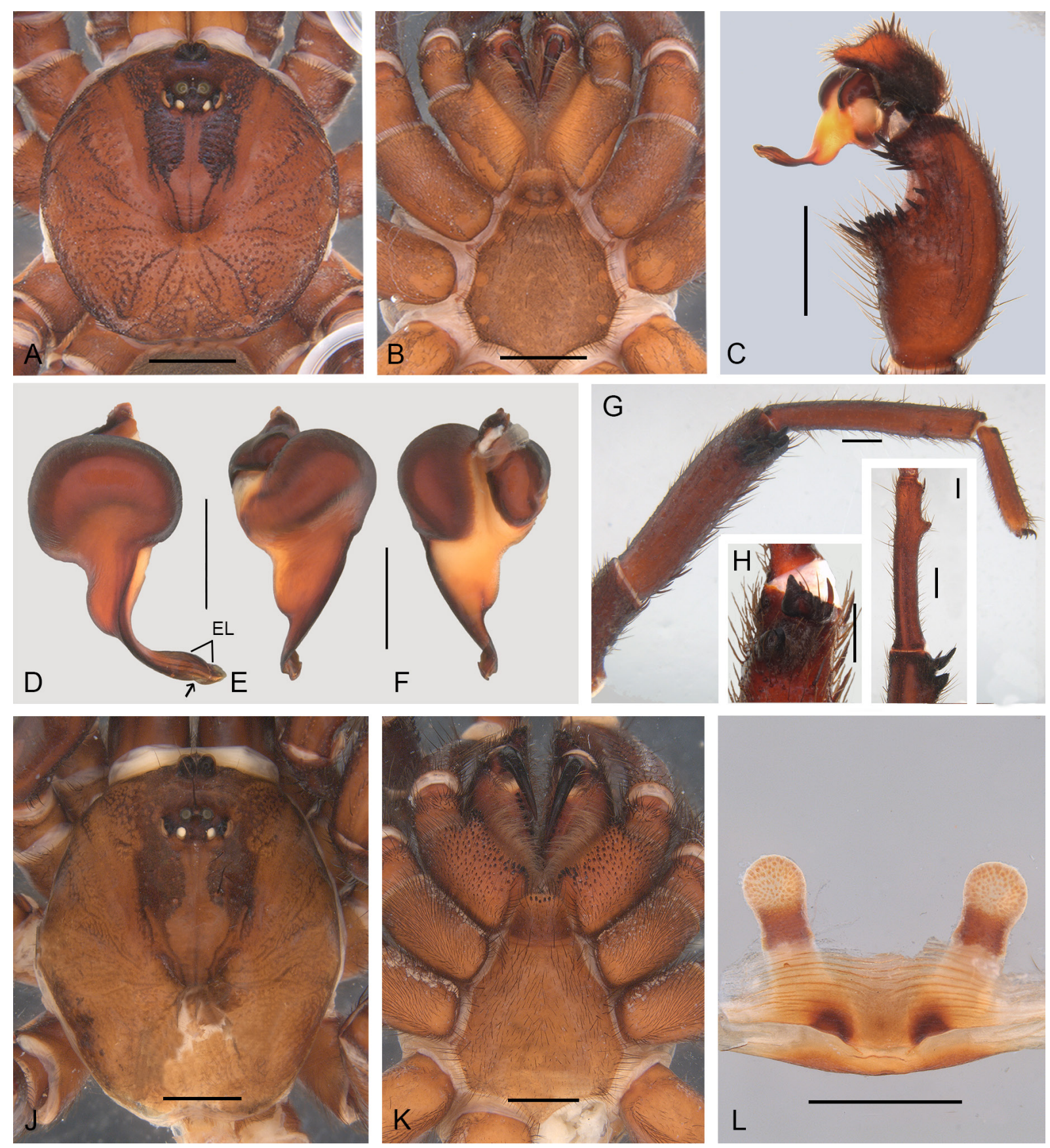

Fig. 27. Idiops pirassununguensis Fukami \& Lucas, 2005. A-I. § (UFMG 607). A. Prosoma (dorsal view). B. Prosoma (ventral view). C. Part of palp (retrolateral view). D-F. Palpal bulb. D. Dorsal view. E. Retrolateral view. F. Prolateral view. G. Tibia, metatarsus and tarsus I (prolateral view). H. Tibial apophysis (prolateral view). I. Tibial apophysis and metatarsus I (dorsal view). J-L. \& (UFMG 11264). J. Prosoma, dorsal view. K. Prosoma, ventral view. L. Genitalia (dorsal view). Abbreviation: EL = embolar lamella. The arrow indicates a constriction. Scale bars $=1 \mathrm{~mm}$. 


\section{Emended description}

Male and female recently described by Fukami \& Lucas (2005). New data on the male and female are included here:

\section{Male (UFMG 607)}

Habitus. See Fig. 27A.

COLOR. Carapace with dark brown spots on anterior half, mainly on cephalic area near eyes (Fig. 27A).

Prosoma. Carapace and ocular arrangement as shown in Fig. 27A. Eye tubercle: 0.6 long; 1 wide. AME-ALE distance 0.8. Basal segment of chelicerae with a prolateral row of 6 large teeth and 4 small retrolateral teeth, grouped in basal half (Fig. 28A); rastellum presenting 12 spines of same size (Fig. 28B).

LeGs. Tibia, metatarsus and tarsus I as shown in Fig. 27G. Pseudoscopula: on tarsus I divided into rows of strong setae; tarsus II-IV totally covered.

PALP. Tibia with expansion of basal half of retrolateral depression (Figs 27C, 28D). Palpal bulb with short embolus with a strongly inclined basal portion and incomplete subapical torsion (Fig. 27D-F).

Spination. Palp: Ti r24, Ta d0-0-4. Leg I: Fe d1-1-2, Ti v1r-1r-2r, Mt 1r-2r-1, Ta r2-1-0. Leg II: Fe d11-2, Ti v0-2r-1r-1, Mt v1r-1r-1r-2, Ta p0-0-1, r2-2-0. Leg III: Fe d1-1-1, Pa d1-1-0, p3-5-8, r0-0-2, Ti v0-1r-0-2, p1-1-4, r1-1-2, Mt v3-4-0-4, p3-2-2, r0-1-2, Ta p0-4-8, r0-4-8. Leg IV: Fe d1-1-2, Pa p16-3-0, Ti v1-2-2, Mt v1-2 -1-3, Ta p2-3-6, r0-0-2.
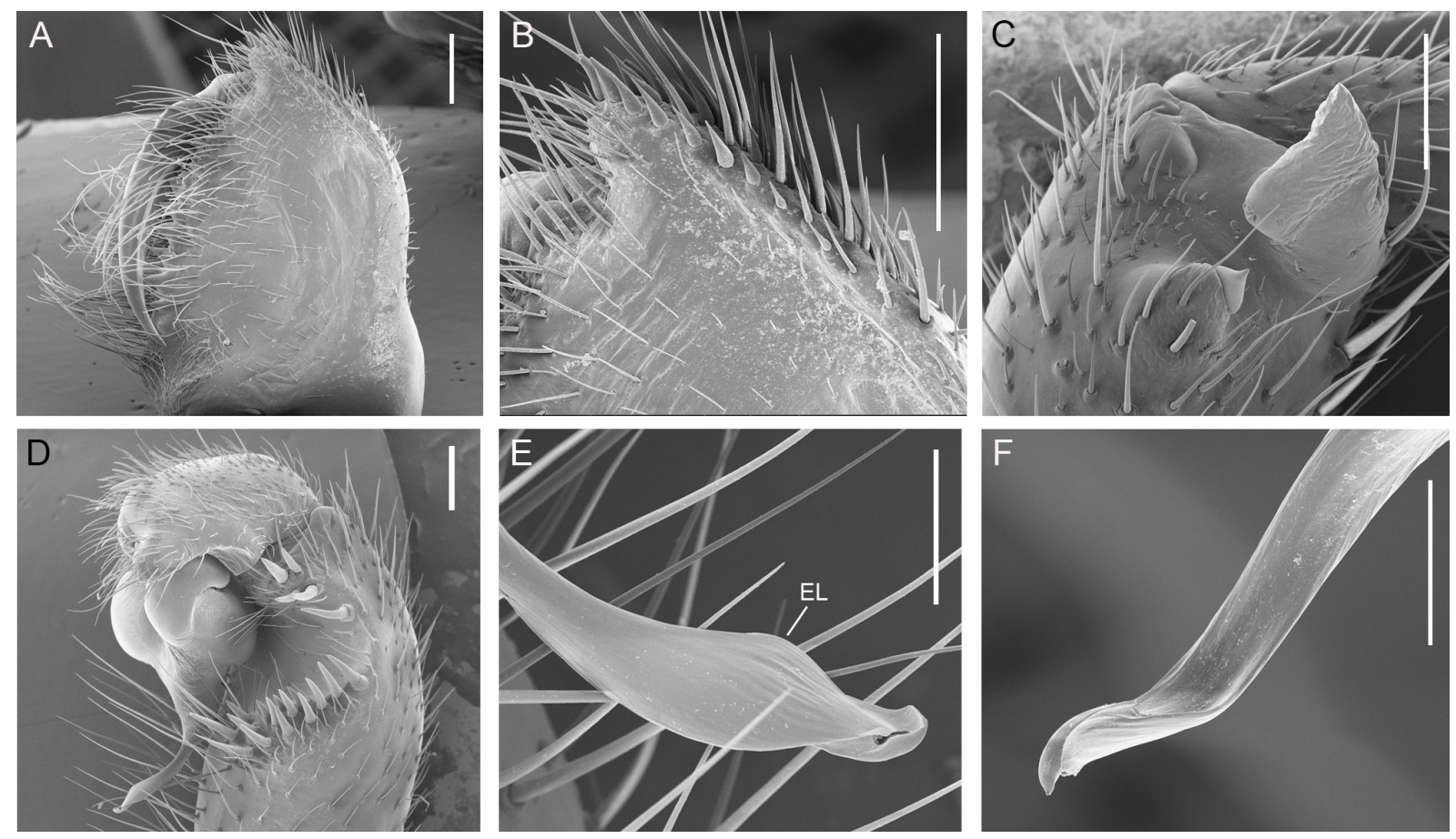

Fig. 28. Scanning electron micrographs of Idiops pirassununguensis Fukami \& Lucas, 2005, ð (MZSP 15651). A. Chelicera (retrolateral view). B. Rastellum (retrolateral view). C. Tibial apophysis (dorsal view). D. Part of the palp, showing retrolateral depression. E-F. Detail of the final portion of the embolus. E. Dorsal view. F. Prolateral view. Abbreviation: $E L=$ embolar lamella. Scale bars: A-D = $0.5 \mathrm{~mm}$; $\mathrm{E}-\mathrm{F}=0.2 \mathrm{~mm}$. 
Female (UFMG 11264)

Habitus. See Fig. 27J.

Measurements. TBL 19.1, CL 9.8, CW 8.8, LL 1.5, LW 1.8, SL 5.4, SW 4.8.

CoLOR. Similar to that of male, except sternum and coxae brownish and dark gray abdomen (Fig. 27J-K).

Prosoma. Carapace and ocular arrangement as shown in Fig. 27J. Eye tubercle: 2.2 long; 1.9 wide. AME-ALE distance 1.4. Eye diameters: AME 0.4, ALE 0.5, PME 0.3, PLE 0.6. Thoracic fovea procurved (Fig. 27J). Labium with 6 cuspules (Fig. 27K). Maxilla with 92 cuspules, distributed over anterior ventral half, with 15 large cuspules at anterior prolateral end and 18 large cuspules at anterior retrolateral end (Fig. 27K). Basal segment of chelicerae with a prolateral row of 6 large teeth and a retrolateral row with 3 small teeth, grouped in basal third, rastellum presenting 22-24 spines of same size (Fig. 27K).

Palp and leg measurements. Palp $=14.4(4.8,3.1,3.2,3.3), \mathrm{I}=16.4(5.3,3.6,3.6,2.6,1.3), \mathrm{II}=14.7$ $(4.8,3.4,2.9,2.4,1.2), \mathrm{III}=15.1(4.1,3.5,2.4,3.1,2), \mathrm{IV}=20.3(5.4,4.1,4.4,4.2,2.2)$.

Spination. Palp: Ti p5-12-13, r7-12-14, Ta v0-0-3, p12-10-8, r12-14-8. Leg I: Ti p5-7-8, r7-8-12, Mt p12-9-11, r11-10-9, Ta v0-0-6, p5-4-2, r5-4-2. Leg II: Ti p3-6-6, r1-3-3, Mt p12-9-12, r5-5-6, Ta v0-0-5, p7-4-3, r6-3-1. Leg III: Pa p3-4-8, r0-0-3, Ti v1-1-1, p2-3-6, r0-2-4, Mt v2-2-2, p6-2-2, r8-1-2, Ta v0-615. Leg IV: Pa p37-4-0, Ti v1-1-2, Mt v2-2-2, Ta v2-4-13.

SPERMATHECAe. Ducts with non-sclerotized basal region, thicker than apical portion; sclerotized apical portion; spherical and non-sclerotized receptacula, with evident granules (Fig. 27L).

\section{Distribution}

Brazil. Widely distributed, with areas in the phytophysiognomies of the Amazon, Caatinga and Cerrado. Records for Central-West region (Distrito Federal and Goiás), North region (Amazonas, Amapá, Pará and Rondônia), Northeast region (Bahia, Maranhão, Paraíba, Piauí and Sergipe) and Southeast region (Minas Gerais and São Paulo) (Fig. 4C).

Idiops rastratus (O. Pickard-Cambridge, 1889)

Figs 4C, 6F, 29-30

Dendricon rastratum O. Pickard-Cambridge, 1889: 250, figs 1-5. Synonymized by Pocock 1895: 223.

Dendricon rastratum - O. Pickard-Cambridge 1890: 623, pl. 53 fig. 2.

Pseudidiops rastratus - Pocock 1895: 223. — Petrunkevitch 1911: 87.

Idiops rastratus - Raven 1985: 139.

\section{Diagnosis}

The male of Idiops rastratus resembles that of I. fuscus by the shape of the palpal bulb, but differs by having the embolus curvature less accentuated (Fig. 29D-F) and the absence of a projection at the base of the retrolateral depression of the palpal tibia. The female differs from that of other species (except Idiops opifex and I. duocordibus sp. nov.) by the strong cuspules on the entire ventral face of the maxillae (Fig. 29K). Differs from I. opifex by the aspect of the spermathecae, with a shorter duct and a slightly larger receptacle compared to the diameter of the duct (Fig. 29L). Differs from I. duocordibus sp. nov. by the unmodified and rounded receptacle (Fig. 29L).

\section{Type material}

Syntypes

BRAZIL - Rio de Janeiro • 4 q o ; Serra dos Órgãos; W. Baker leg.; BMNH 1890.1.20.1 to 1890.1.20.4. 


\section{Other material examined}

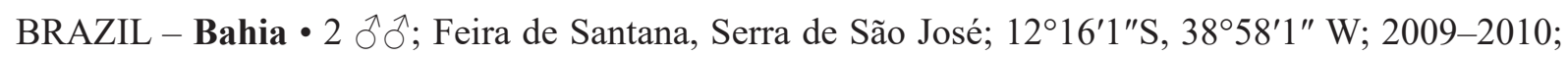
G. de Ferreira leg.; IBSP 258656 to $258657 \cdot 1$ q, 1 juv.; Porto Seguro, Arraial d'Ajuda; $16^{\circ} 27.643^{\prime}$ S,
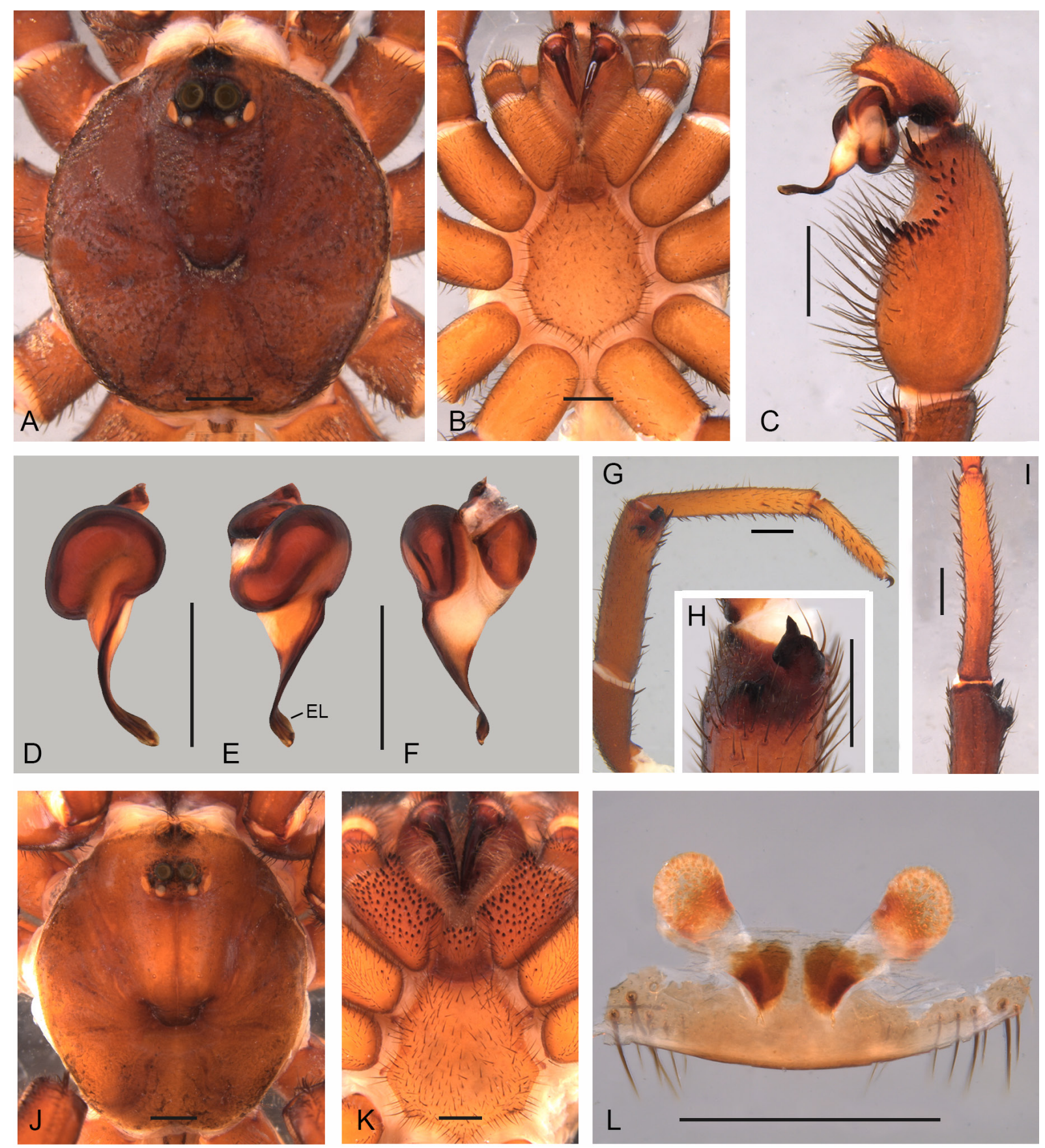

Fig. 29. Idiops rastratus (O. Pickard-Cambridge, 1889). A-I. Ø̊ (MZSP 24282). A. Prosoma (dorsal view). B. Prosoma (ventral view). C. Part of palp (retrolateral view). D-F. Palpal bulb. D. Dorsal view. E. Retrolateral view. F. Prolateral view. G. Tibia, metatarsus and tarsus I (prolateral view). H. Tibial apophysis (prolateral view). I. Tibial apophysis and metatarsus I (dorsal view). J-L. ㅇ (MZSP 24282). J. Prosoma (dorsal view). K. Prosoma (ventral view). L. Genitalia (dorsal view). Abbreviation: EL = embolar lamella. Scale bars $=1 \mathrm{~mm}$. 


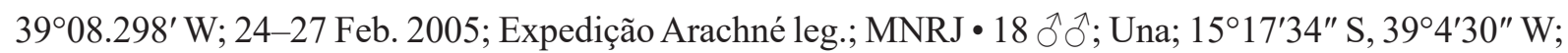
Dec. 1999; K. Kato leg.; MZSP 24233 • 11 ôें ; same collection data as for preceding; MZSP 24218

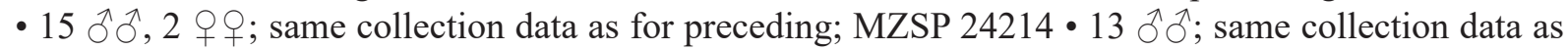

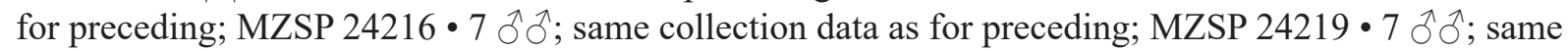
collection data as for preceding; MZSP 24221 - 10 ô; same collection data as for preceding; MZSP $24292 \cdot 7 \hat{\jmath}$; same collection data as for preceding; MZSP 24211 • $2 \hat{\jmath} \widehat{\partial}, 1$ क ; same collection data as

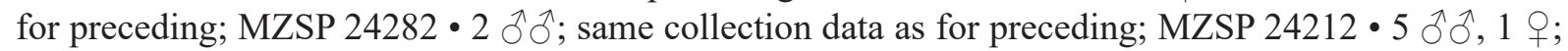
same collection data as for preceding; MZSP $24210 \bullet 6 \hat{\delta}$; same collection data as for preceding; MZSP $24284 \cdot 3 \widehat{\partial} \widehat{\partial}(\mathrm{SEM})$; same collection data as for preceding; MZSP $24215 \cdot 6 \hat{\partial}$; ; same collection data as for preceding; MZSP 24675 • $5 \hat{\delta} \widehat{\delta}$; same collection data as for preceding; MZSP $24220 \bullet 3 \hat{\partial}$; same

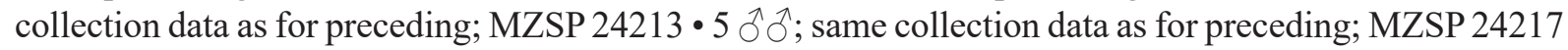

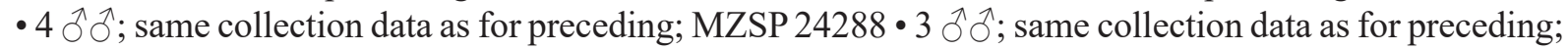

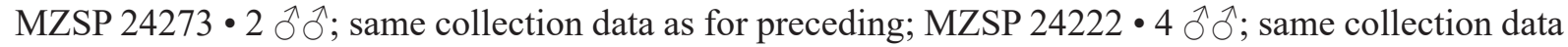

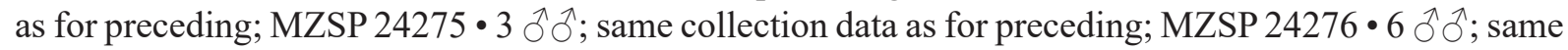
collection data as for preceding; MZSP 24287. - Minas Gerais • 1 đ̊; Nova Lima, Serra da Moeda, Cave PBR_10_11; 2009'20" S, 4358'24" W; 15-20 Mar. 2010; R. Bessi leg.; IBSP 196034. - Espírito Santo

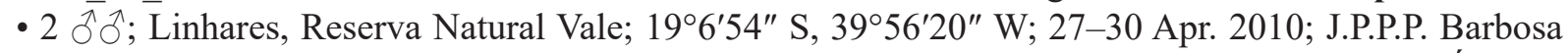
et al. leg.; IBSP 234713. - Rio de Janeiro • 1 \%; Teresópolis, Parque Nacional da Serra dos Órgãos; $23^{\circ} 27^{\prime} 33^{\prime \prime}$ S, 46 $6^{\circ} 38^{\prime} 2^{\prime \prime}$ W; 10-22 Nov. 2010; R.P. Indicatti and F.U. Yamamoto leg.; IBSP 243973. - São

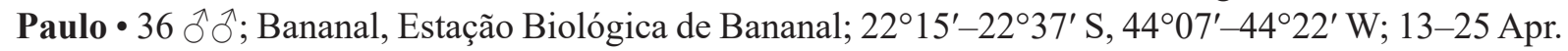

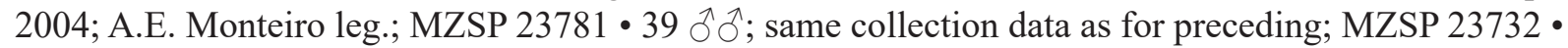
1; Bananal, Estação Biológica de Bananal; 2010; J.M. Pereira leg.; IBSP 245408.
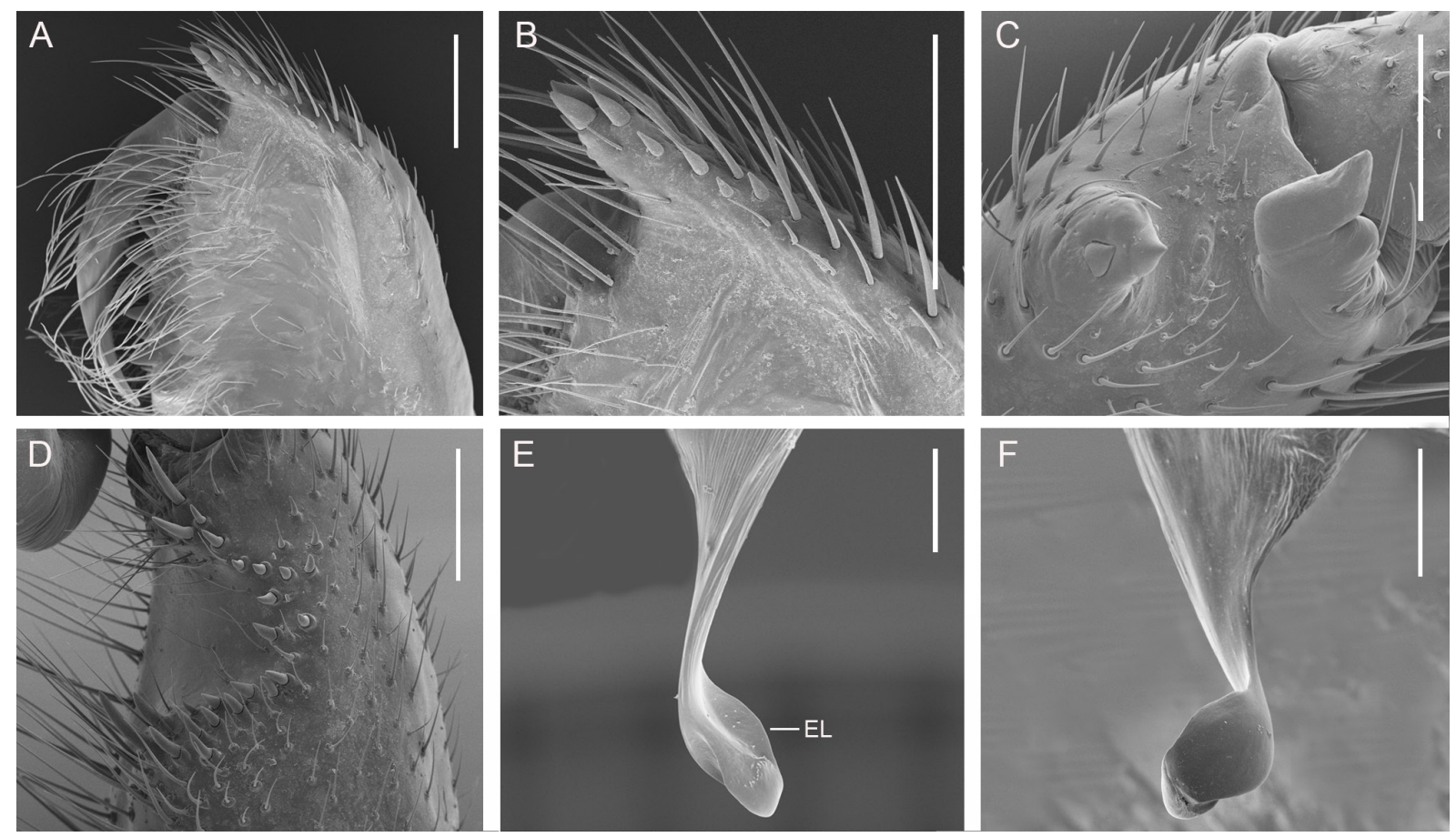

Fig. 30. Scanning electron micrographs of Idiops rastratus (O. Pickard-Cambridge, 1889), § (MZSP 24215). A. Chelicera (retrolateral view). B. Rastellum (retrolateral view). C. Tibial apophysis (dorsal view). D. Part of the palp, showing retrolateral depression. E-F. Detail of the final portion of the embolus. E. Retrolateral view. F. Prolateral view. Abbreviation: $E L=$ embolar lamella. Scale bars: $\mathrm{A}-\mathrm{D}=0.5 \mathrm{~mm} ; \mathrm{E}-\mathrm{F}=0.2 \mathrm{~mm}$. 


\section{Description}

Male (MZSP 24282)

Habitus. See Fig. 29A.

MeAsurements. TBL 11.2, CL 5.5, CW 5.2, LL 0.7, LW 1, SL 3, SW 2.6.

Color. Carapace and legs reddish brown, with yellowish coxae, brownish sternum (Fig. 29A-B), abdomen dorsally dark gray and ventrally brownish.

Prosoma. Carapace and ocular arrangement as shown in Fig. 29A. Eye tubercle: 1.3 long; 1.4 wide. Distance AME-ALE 0.5. Eye diameters: AME 0.5, ALE 0.3, PME 0.2, PLE 0.4. Thoracic fovea procurved (Fig. 27A). Labium and sternum without cuspules (Fig. 29B). Basal segment of chelicerae with a prolateral row of 5 large teeth and 4-5 small retrolateral teeth in basal third (Fig. 30A), with proeminent rastellum, with 7-8 spines, with larger ones in distal region (Fig. 30B).

LeGs. Tibia, metatarsus and tarsus I as shown in Fig. 29G. Basal branch of tibial apophysis with additional tooth (Fig. 30C). Pseudoscopula: tarsus I smooth, II weakly covered, III-IV densely covered.

PALP. Tibia with conspicuous retrolateral depression and with larger spines concentrated on basal half (Figs 29C, 30D); embolus with apical lamella with a differentiated dorsal and ventral border (Figs 29D-F, 30E-F).

Palp and Leg measurements. Palp $=9(3.2,1.6,3,1.2), \mathrm{I}=20.7(6.4,3,4.9,4.4,2), \mathrm{II}=16,5$ (5.3, 2 , $3.6,3.8,1.8), \mathrm{III}=15.13(4.1,2.1,3,3.9,1.9), \mathrm{IV}=21.7(6,2.7,5.3,5.3,2.4)$.

SPInATion. Palp: Ti r36, Ta d-0-0-4. Leg I: Ti v1r-1r-2r-1ap, Mt v35-3, Ta v45, p2-3-3, r4-3-1. Leg II: Ti v1r-1r-3r-2, Mt v4-6-5-3ap, p0-1-1, Ta p3-4-2, r3-3-4. Leg III: Pa p1-2-7, r0- 1-2, Ti v1-2-2-1ap, p1-1-1, r1-1-1, Mt v2-4-3-4, p0-0-2, r1-1-1, Ta p2-3-2, r3-4-2. Leg IV: Pa p6-1-0, Ti v1-1-4, Mt v1-1r-1-3, Ta $\mathrm{p} 2-1-3, \mathrm{r} 0-1-2$.

Female (BMNH 1890.1.20.1)

Habitus. See Fig. 29J.

MeAsurements. TBL 13, CL 5.8, CW 4.9, LL 0.8, LW 2, SL 3.5, SW 3.5.

CoLOR. Carapace and legs reddish brown, with yellowish coxae, brownish sternum (Fig. 29J-K), abdomen dorsally dark gray and ventrally brownish.

Prosoma. Carapace and ocular arrangement as shown in Fig. 29J. Eye tubercle: 1.7 long; 1.5 wide. Distance AME-ALE 0.9. Eye diameters: AME 0.5, ALE 0.4, PME 0.3, PLE 0.4. Thoracic fovea procurved (Fig. 29J). Labium with 14 cuspules. Maxilla with 113 cuspules, distributed throughout ventral region (Fig. 29K). Basal segment of chelicerae with a prolateral row of 5 large teeth and a retrolateral row with 9 small teeth, grouped in basal third, rastellum with 12-14 spines, distal ones longer.

PalP And leg measurements. Palp $=11.4(3.9,2.4,2.7,2.4), \mathrm{I}=12.7(4.4,2.7,2.7,1.9,1), \mathrm{II}=10.8$ $(3.3,2.5,2.2,1.8,1), \mathrm{III}=11.4(3.4,2.4,1.9,2.3,1.4), \mathrm{IV}=16.2(4.8,2.9,3.6,3.1,1.8)$.

Spination. Palp: Ti r36, Ta d-0-0-4. Leg I: Ti v1r-1r-2r-1ap, Mt v35-3, Ta v45, p2-3-3, r4-3-1. Leg II: Ti v1r-1r-3r-2ap, Mt v4-6-5-3, p0-1-1, Ta p3-4-2, r3-3-4. Leg III: Pa p1-2-7, r0-1-2, Ti v1-2-2, p1-1-1, r1-1-1, Mt v2-4-3-4, p0-0-2, r1-1-1, Ta p2-3-2, r3-4-2. Leg IV: Pa p6-1-0, Ti v1-1-4, Mt v1-1r-1-3, Ta p2-1-3, r0-1-2 
SPERmatheCAe. Base divided by non-sclerotized area. Ducts short and slightly narrower than receptacles, which are spherical and with evident granules (Fig. 29L).

\section{Distribution}

Brazil. Mainly distributed in Atlantic Forest phytophysiognomies along the coastal zone, with records for the Southeast region (east of São Paulo, Espírito Santo and Rio de Janeiro) and Northeast region (center-south of Bahia). Only one record for the central region of Minas Gerais (Fig. 4C)

Idiops rohdei Karsch, 1886

Figs $3 \mathrm{C}, 31$

Idiops rohdei Karsch, 1886: 93.

\section{Diagnosis}

The male of Idiops rohdei differs from that of other Neotropical species by the rectangular shape in dorsal view of the apical branch of the tibial apophysis (Fig. 31H) and by the short metatarsus I with a projection on the apical half (Fig. 31I). The female differs in having the spermathecae with ducts slightly curved outward in the median portion and elongated bean-shaped receptacles (Fig. 31J-K).

\section{Type material}

\section{Holotype}

PARAGUAY • \%; R. Rohde leg.; ZMB 6230.

\section{Other material examined}

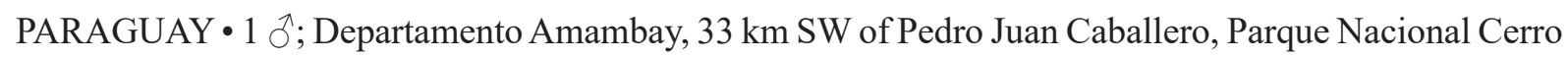
Corá, camping area; $22^{\circ} 38^{\prime} 04.6^{\prime \prime} \mathrm{S}, 56^{\circ} 01^{\prime} 33.8^{\prime \prime}$ W; 22 Oct. 2019; A. Pérez-González, A. Ojanguren, D.J. Guerrero and J. Kochalka leg.; IBNP-2444.

Remark: The description of Idiops rohdei was based on a female specimen from Paraguay, without any further details about its locality. We had access to a male specimen from Parque Nacional Cerro Corá, mid-eastern Paraguay. The female holotype and the male specimen share the overall body coloration, especially close to the eye tubercle, which is dark brown. We decided to consider them as cospecifics, pending the availability of more specimens from Paraguay or nearby regions to support our decision.

\section{Description}

Male (IBNP-2444)

Habitus. See Fig. 31A.

MeAsurements. TBL 6.3, CL 3.3, CW 3.1, LL 0.5, LW 0.6, SL 1.8, SW 1.7.

CoLor. Carapace and legs reddish brown, with dark brown ocular tubercle, yellowish coxae, brownish sternum (Fig. 31A-B), abdomen dorsally dark gray and ventrally brownish.

Prosoma. Carapace and ocular arrangement as shown in Fig. 31A. Eye tubercle: 0.6 long; 0.8 wide. Distance AME-ALE 0.5. Eye diameters: AME 0.3, ALE 0.3, PME 0.1, PLE 0.2. Thoracic fovea procurved (Fig. 31A). Labium and sternum without cuspules (Fig. 31B). Basal segment of chelicerae with a prolateral row of 4 larger teeth, and a small, retrolateral row with 2 small teeth, grouped in basal half, rastellum with 3 spines (Fig. 31B). 

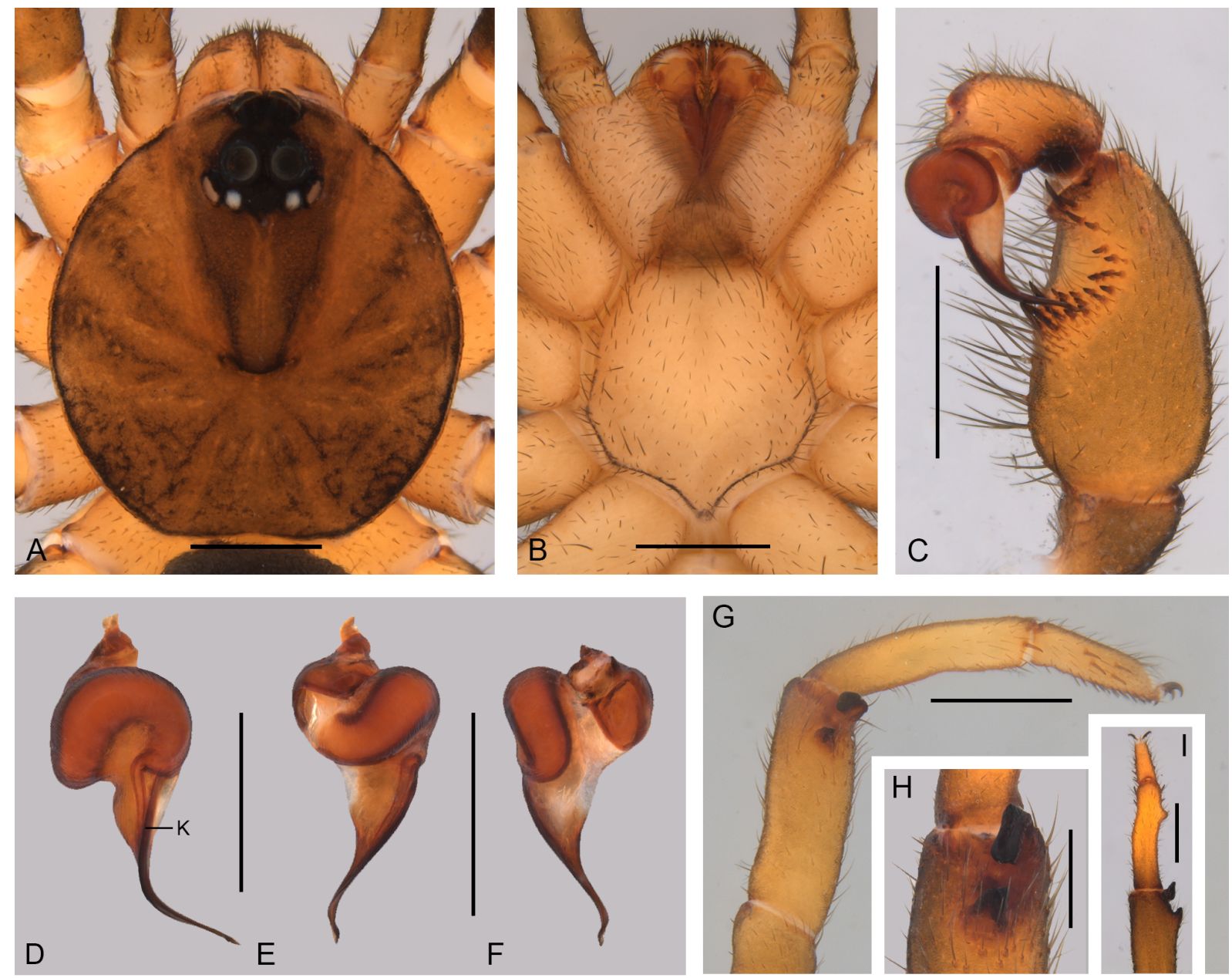

\section{C}
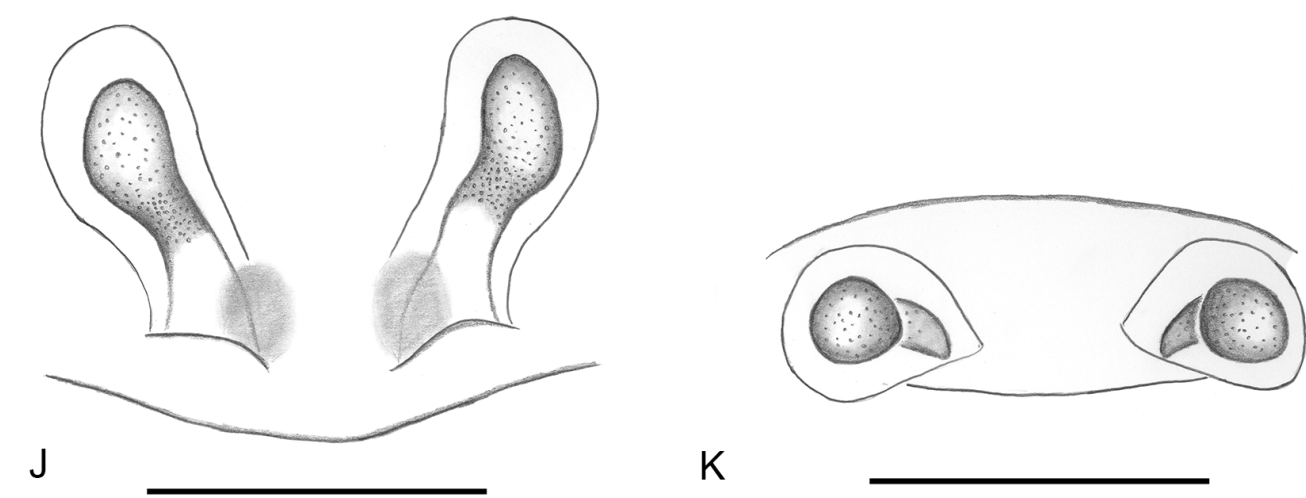

Fig. 31. Idiops rohdei Karsch, 1886. A-I. Ø̊ (IBNP-2444). A. Prosoma (dorsal view). B. Prosoma (ventral view). C. Part of palp (retrolateral view). D-F. Palpal bulb. D. Dorsal view. E. Retrolateral view. F. Prolateral view. G. Tibia, metatarsus and tarsus I (prolateral view). H. Tibial apophysis (prolateral view). I. Tibial apophysis and metatarsus I (dorsal view). J-K. , genitalia, holotype (ZMB 6230). J. Dorsal view. K. Frontal view. Abbreviation: $\mathrm{K}=$ keel. Scale bars $=1 \mathrm{~mm}$. 
LEGS. Tibia, metatarsus and tarsus I as shown in Fig. 31G. Pseudoscopula: tarsus II-IV fully covered.

PALP. Tibia with spines distributed along margin of retrolateral depression, with larger spines on basal and apical portions (Fig. 31C), embolus with a keel in dorsal and retrolateral view, embolus strongly curved dorsally with abrupt reduction in thickness in its apical half, incomplete torsion at spermatic duct opening (Fig. 31D-F).

PalP and Leg measurements. Palp $=2.8(1,0.5,0.9,0.4), \mathrm{I}=4.2(1.4,0.7,1,0.8,0.3), \mathrm{II}=3.5(1,0.6$, $1,0.6,0.3), \mathrm{III}=4.1(1.5,0.6,0.7,0.8,0.5), \mathrm{IV}=4.4(1,0.7,1.2,1,0.5)$.

SPination. Palp: Ti r25. Leg I: Fe d1-2-2, Pa v0-0-1, Ti v1-1-2, p0-0-1, r2-2-2, Mt v1-1-1, p0-0-1, r22-2, Ta v3-6-5, p0-1-1, r2-2-1. Leg II: Pa v0-0-2, Ti v2-1-3, r1-2-2, Mt v4-3-4, p0-0-3, r-2-2, Ta p0-1-1, r2-1-1. Leg III: Pa p0-0-3, Ti v0-2-2, Mt v0-1-3, p0-0-1, Ta r1-0-1. Leg IV: Pa p0-0-6, Ti p0-0-2, Mt v0-2-4, Ta p1-1-2.

Female (holotype ZMB 6230)

Measurements. TBL 13.9, CL 6.8, CW 5.8, LL 1, LW 1.4, SL 4.2, SW 3.7.

CoLOR. Carapace and legs red brown. Eye tubercle dark brown. Sternum brown.

Prosoma. Eye tubercle: 1.7 long; 1.5 wide. AME-ALE distance 1. Eye diameters: AME 0.4, ALE 0.3, PME 0.3, PLE 0.4. Thoracic fovea procurved. Labium with 5 cuspules. Maxilla with 58 cuspules, distributed throughout anterior ventral half. Basal segment of chelicerae with a prolateral row of 6 large teeth and 11 small retrolateral teeth, grouped in median area.

Palp and leg measurements. Palp $=11.2(3.7,2.3,2.6,2.6), \mathrm{I}=13.1(4.4,2.9,2.8,2,1), \mathrm{II}=11.7$ (4, $2.7,2.2,1.8,1), \mathrm{III}=12.6(3.8,2.7,1.8,2.6,1.7), \mathrm{IV}=17.3(4.9,3.3,3.7,3.4,2)$.

SinAtion. Palp: Fe p0-0-2, Pa p0-1-0, Ti p7-7-12, r6-11-12, Ta v6-11-12. Leg. I: Ti p6-5-8, r4-7-15, Mt p10-7-7, r11-8-7, Ta v0-0-7, p4-4-4, r4-4-0. Leg II: Ti p3-4-7, r1-2-4, Mt p13-7-9, r5-5-5, Ta v0-0-6, p55-3, r5-3-0. Leg III: Pa p6-5-12, r0-0-3, Ti v0-0-2, p0-4-8, r0-3-3, Mt v2-2-3, p10-4-3, r5-2-1, Ta v4-8-9. Leg IV: Pa p39-0-0, Ti v1-1-1, Mt v1-1-7, r0-0-1, Ta v4-6-11.

SPERMATHECAE. Bean-shaped receptacula with conspicuous granules, long, translucent ducts (Fig. 31J-K).

\section{Distribution}

Paraguay. Departamento Amambay (Fig. 3C).

Idiops sertania Fonseca-Ferreira, Guadanucci \& Brescovit sp.nov. urn:lsid:zoobank.org:act:8C6CC368-27DF-45A0-9164-2CA32C4D0F39

Figs $4 \mathrm{C}, 32-33$

\section{Diagnosis}

Males of Idiops sertania sp. nov. differ from those of other Neotropical species by having the robust embolus with a wide basal half, strongly curved dorsally, with torsion at the apical end and a conspicuous embolar lamella close to the opening of the sperm duct (Figs 32D-F, 33E-F) and by having the palpal tibia elongated (similar to I. germaini) (Fig. 32C). Differs from I. germaini by having large spines distributed along the margin of the retrolateral depression (Figs 32C, 33D) and by the robust tibial apophysis (Fig. 32H). 


\section{Etymology}

The term 'sertania', referenced in the specific epithet, is a tribute to the 'sertão' of Brazil, a large region of the country where these spiders occur. The term sertão refers to culturally diverse regions, far from the coast and large cities, and generally associated with rural regions with little human occupation.

\section{Type material}

\section{Holotype}

BRAZIL - Minas Gerais • ${ }^{\top}$; Januária, Parque Nacional Cavernas do Peruaçu; $15^{\circ} 10^{\prime} 0^{\prime \prime} \mathrm{S}, 44^{\circ} 22^{\prime} 0^{\prime \prime} \mathrm{W}$; 3-25 Jan. 2008; M.T. Junior and R.S. Recoder leg.; IBSP 15348.

\section{Paratypes}

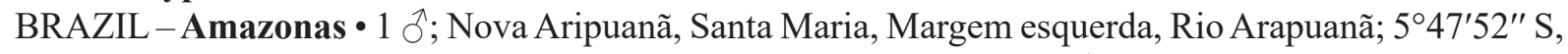
60¹5'55" W; May 2002; M. Trefaut leg.; MZUSP 21511. - Piauí • 1 đ̊; Guaribas, Parque Nacional da Serra das Confusões; 9¹3'16" S, 43²9'21" W; Oct. 2006; P.R.R.Silva et al. leg.; CHNUFPI 4031. -
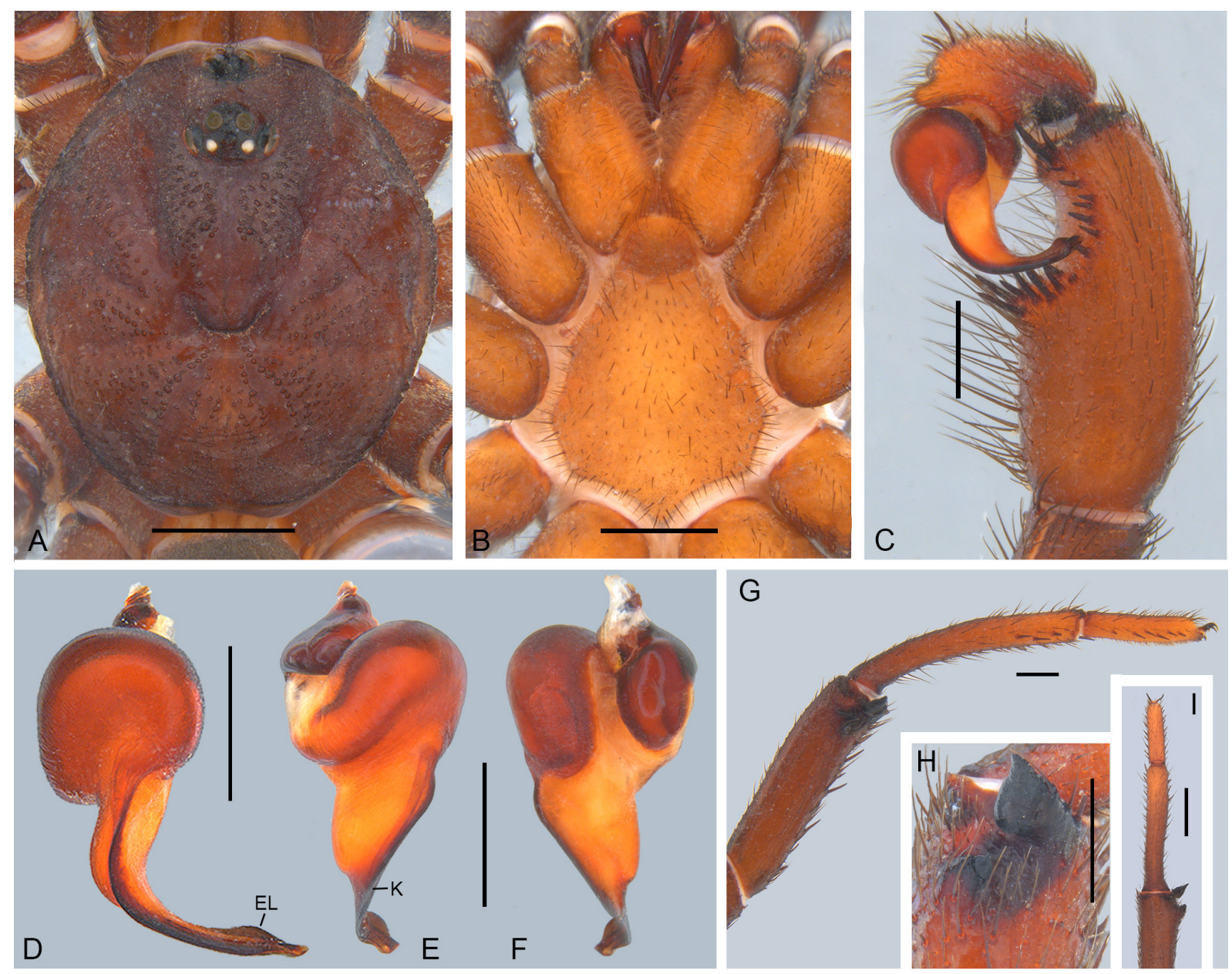

Fig. 32. Idiops sertania Fonseca-Ferreira, Guadanucci \& Brescovit sp. nov., đ̂, holotype (IBSP 15348). A. Prosoma (dorsal view). B. Prosoma (ventral view). C. Part of palp (retrolateral view). D-F. Palpal bulb. D. Dorsal view. E. Retrolateral view. F. Prolateral view. G. Tibia, metatarsus and tarsus I (prolateral view). H. Tibial apophysis (prolateral view). I. Tibial apophysis and metatarsus I (dorsal view). Abbreviations: $\mathrm{EL}=$ embolar lamella; $\mathrm{K}=$ keel. Scale bars $=1 \mathrm{~mm}$. 
Ceará • 1 đ’; Crato, Floresta Nacional do Araripe; 07²7'07.3" S, 39¹9'52.4" W; 2018; R. Azevedo leg.; IBSP 227617. - Minas Gerais • 1 §̊; same collection data as for holotype; IBSP 15346.

\section{Other material examined}

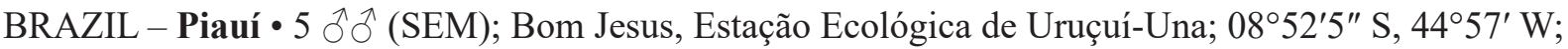
19-29 Jan. 2001; G.G. Martinelle leg.; MZSP 21546 • 2 ふో ô; União, Campus COMVAPI Ltda; $4^{\circ} 35^{\prime} 9^{\prime \prime}$ S,

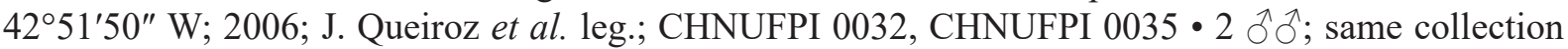
data as for preceding; CHNUFPI $0037 \cdot 3 \hat{\jmath}$; same collection data as for preceding; CHNUFPI 0034

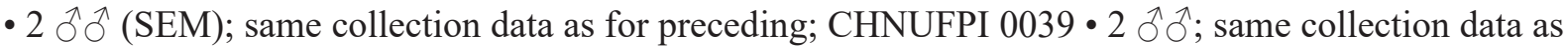
for preceding; CHNUFPI 0033 • 1 万'; same collection data as for preceding; $4^{\circ} 38^{\prime} 6^{\prime \prime} \mathrm{S}, 42^{\circ} 50^{\prime} 19^{\prime \prime} \mathrm{W}$;

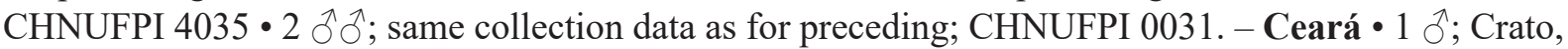

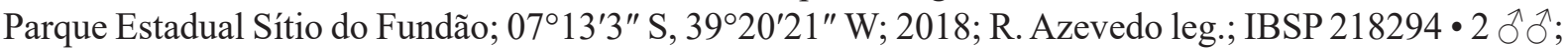
Crato, Floresta Nacional do Araripe; $07^{\circ} 27^{\prime} 07.3^{\prime \prime}$ S, 39¹9'52.4" W; 2018; R. Azevedo leg.; IBSP 218307 ,

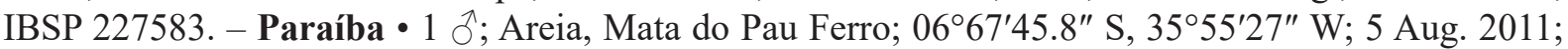
S.B.R. Alencar et al. leg.; CHNUFPI 0041. - Bahia • 1 ; ; Contendas do Sincorá, Floresta Nacional Contendas do Sincorá; $13^{\circ} 55^{\prime} 08,7^{\prime \prime}$ S, $41^{\circ} 07^{\prime} 13,8^{\prime \prime}$ W; 12-14 Jan. 2012; I.L.F. Magalhães et al. leg.; IBSP

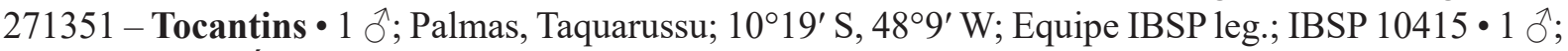
Porto Nacional, Área urbana, UHE Luiz Eduardo Magalhães; $10^{\circ} 42^{\prime} 28^{\prime \prime}$ S, $48^{\circ} 25^{\prime} 1^{\prime \prime}$ W; Equipe IBSP leg.;

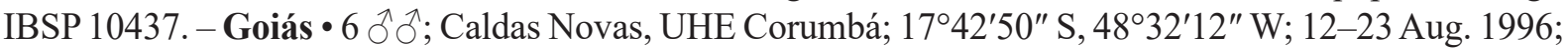
M.T.I. Rodrigues et al. leg.; MZSP 15656 - 2 o $^{\prime}$; Colinas do Sul, Serra da Mesa; $14^{\circ} 01^{\prime}$ S, $48^{\circ} 12^{\prime}$ W; 2-15 Dec. 1995; Silvestri, Dietz and Campaner leg.; MZSP 28851 • 1 đ̊; Catalão, Barragem, Serra do Facão; 17³1'51.07" S, 47³3'29.57" W; Jul. 2013; R.B. Martines and R.M.C. Silveira leg.; UFMG 4442

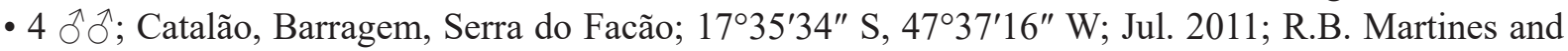
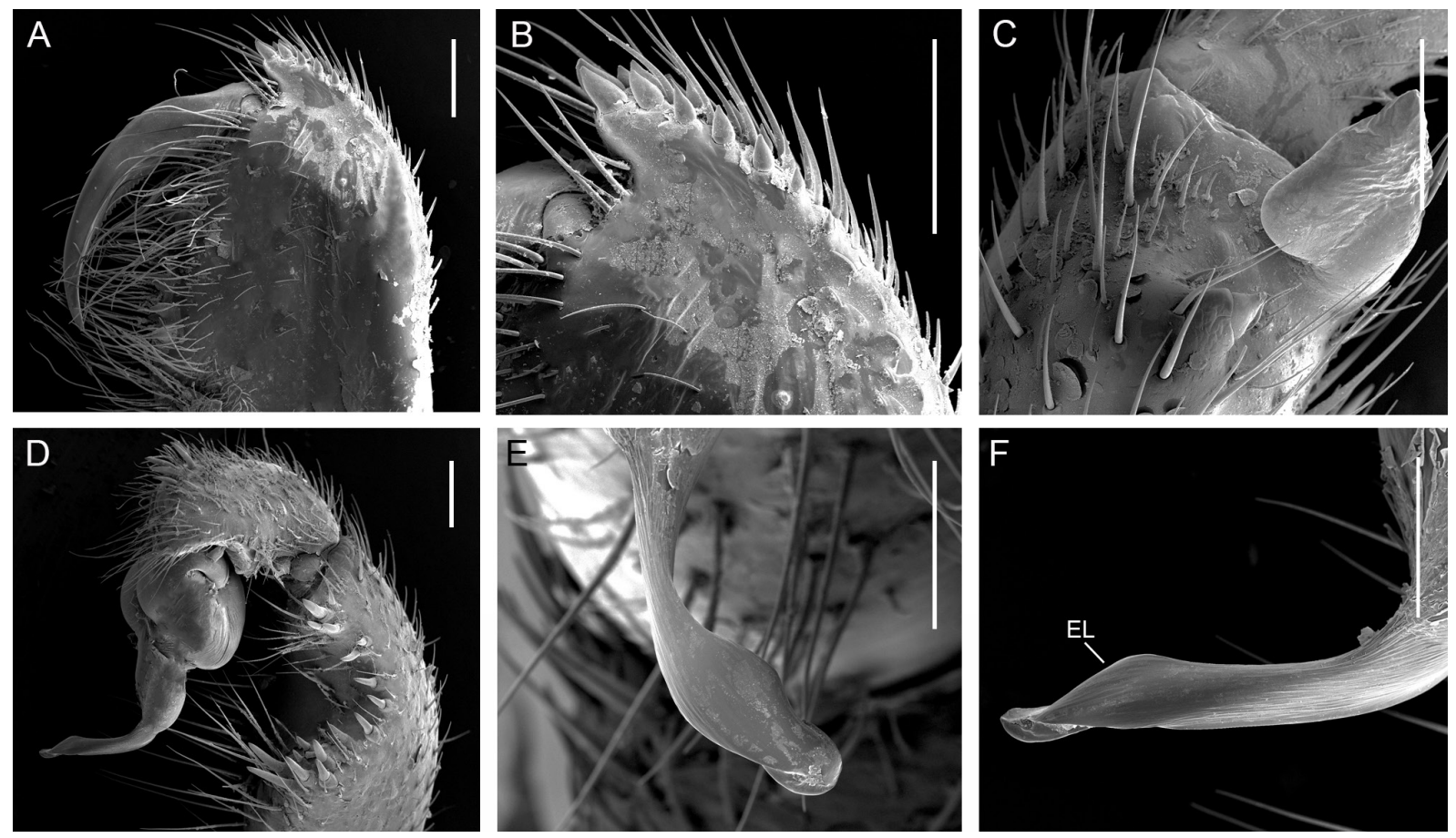

Fig. 33. Scanning electron micrographs of Idiops sertania Fonseca-Ferreira, Guadanucci \& Brescovit sp. nov., $\widehat{\partial}$ (CHNUFPI 0039). A. Chelicera (retrolateral view). B. Rastellum (retrolateral view). C. Tibial apophysis (dorsal view). D. Part of the palp, showing retrolateral depression. E-F. Detail of the final portion of the embolus. E. Retrolateral view. F. Prolateral view. Abbreviation: EL = embolar lamella. Scale bars: A-D $=0.5 \mathrm{~mm}$; E-F $=0.3 \mathrm{~mm}$. 


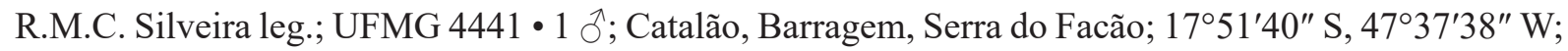
Jul. 2012; R.B. Martines and R.M.C. Silveira leg. UFMG 4440. - Mato Grosso - 1 o ; Chapada dos

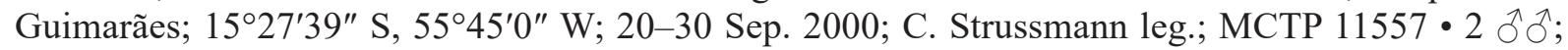
Chapada dos Guimarães; 20-29 Sep. 2000; C. Strussmann leg.; MCTP 13614 • 2 ふ઼; Chapada dos

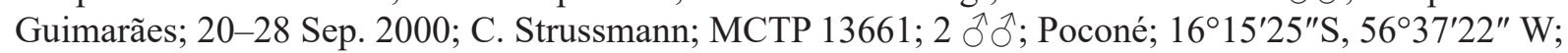
2010-2012; D. Batistella leg., Winkler extractor; IBSP 249196. - Mato Grosso do Sul • 1 ð’; Três

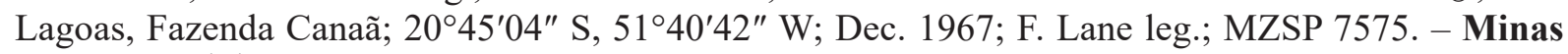
Gerais • $2 \overbrace{}^{\lambda} \partial^{\circledR}$; Januária, Parque Nacional Cavernas do Peruaçu; $15^{\circ} 10^{\prime} 0^{\prime \prime} \mathrm{S}, 44^{\circ} 22^{\prime} 0^{\prime \prime} \mathrm{W}$; 3-25 Jan. 2008; M. Teixeira-Junior and R.S. Recoder leg.; IBSP 15345, IBSP $15347 \cdot 2$ ठें; same collection data as for preceding; IBSP 210881.

\section{Description}

Male (holotype IBSP 15348)

Habitus. See Fig. 32A.

MeAsurements. TBL 15.6, CL 7.7, CW 6.9, LL 1, LW 1.3, SL 4, SW 3.7.

CoLOR. Dark brown carapace, reddish brown legs, light brown sternum, abdomen gray (Fig. 32A-B).

Prosoma. Carapace and ocular arrangement as shown in Fig. 32A. Eye tubercle: 0.9 long; 1.2 wide. AME-ALE distance 1. Eye diameters: AME 0.2, ALE 0.4, PME 0.2, PLE 0.5. Thoracic fovea procurved (Fig. 32A). Labium and sternum without cuspules (Fig. 32B). Basal segment of chelicerae with a prolateral row of 6 large teeth and 6 small retrolateral teeth, grouped in the basal half, proeminent rastellum with 11-12 spines, distals largest (Fig. 33A-B).

LEGs. Tibia, metatarsus and tarsus I as shown in Fig. 32G. Leg I with tibial apophysis with apical branch twice the size of basal branch (Figs 32H-I, 33C). Pseudoscopula: tarsus I weakly covered, II-IV totally covered.

PALP. Tibia with conspicuous retrolateral depression and large spines distributed along its entire length (Figs 32C, 33D); embolus with keel in retrolateral view (Fig. 32E).

PalP and leg measurements. Palp $=10.2(2.4,2.2,3.9,1.7), \mathrm{I}=23.6(7.2,3.3 .4 .9,5.4,2.8), \mathrm{II}=20.8$ $(6.4,2.9,4.4,4.8,2.3), \mathrm{III}=19.3(5.3,2.5,3.6,5,2.9), \mathrm{IV}=27(7.1,3.4,6,7,3.5)$.

Spination. Palp: Ti r20, Ta d0-0-2. Leg I: Pa v4, Ti v4-5-4, Mt r1-1-4, p0-0-4, Ta r3-4-3, p1-3-2. Leg II: Pa p0-0-4, r0-0-3, Ti v6-5-7, Mt p0-2-3, r2-3-4, Ta p2-2-3, r4-3-4. Leg III: Pa p1, Ti v1-2-2, p0-0-1, Mt v2-4-5, p2-2-2, Ta r4-4-5. Leg IV: Pa v0-0-2, Ti v1-1-3, Mt v9-9-11, d1-1-2, r0-0-3, p0-1-2, Ta p3-3-7, $\mathrm{r} 0-0-2$.

\section{Distribution}

Brazil. Widely distributed, with areas covering the phytophysiognomies of the Amazon, Caatinga and Cerrado. Records for Central-west region (Goiás, Mato Grosso and Mato Grosso do Sul), North region (Amazonas), Northeast region (Bahia, Ceará and Piauí) and Southeast region (northern part of Minas Gerais) (Fig. 4C).

\section{Idiops siolii (Bücherl, 1953)}

Figs 3B, 34

Pseudidiops siolli Bücherl, 1953: 126, figs 6-8.

Idiops siolii - Raven 1985: 158. 


\section{Diagnosis}

The female of Idiops siolii differs from that of other Neotropical species by having the spermathecae with ducts with a thickened median portion and mushroom-shaped receptacles (Fig. 34C).

\section{Type material}

\section{Holotype}

BRAZIL - Pará • +; Belém, Campus do IAN - Embrapa Amazônia Oriental; IBSP 3123. Lost in the fire of 2010.

Neotype (here designated)

BRAZIL - Pará • क; Melgaço, Flona Caxiuanã, Estação Científica Ferreira Penna, Plote B; $1^{\circ} 47^{\prime} 32.3^{\prime \prime}$ S, 51²6'2.5" W; 6-11 Apr. 2002; MPEG 1012.

Remark: The type specimen of Idiops siolii was lost in the 2010 IBSP fire. In accordance with the criteria of the ICZN Code (ICZN 1999), a neotype is designated here, because the type is lost and the original description is inadequate to stabilise the species. The neotype is a specimen collected near the type locality, in the state of Pará, Brazil.

\section{Description}

Male

Unknown.

Female (neotype MPEG 1012)

Habitus. See Fig. 34A.

Measurements. TBL 13.8, CL 7.6, CW 6.6, LL 1.1, LW 1.5, SL 4.4, SW 3.7.

CoLor. Carapace and legs reddish brown, with yellowish coxae, reddish brown sternum (Fig. 34A-B). Abdomen gray.

Prosoma. Carapace and ocular arrangement as shown in Fig. 34A. Eye tubercle: 0.7 long; 1.4 wide. AME-ALE distance 1. Eye diameters: AME 0.21, ALE 0.5, PME 0.2, PLE 0.4. Thoracic fovea procurved. Labium with 3 cuspules (Fig. 34B). Maxilla with 31 cuspules, distributed throughout anterior ventral
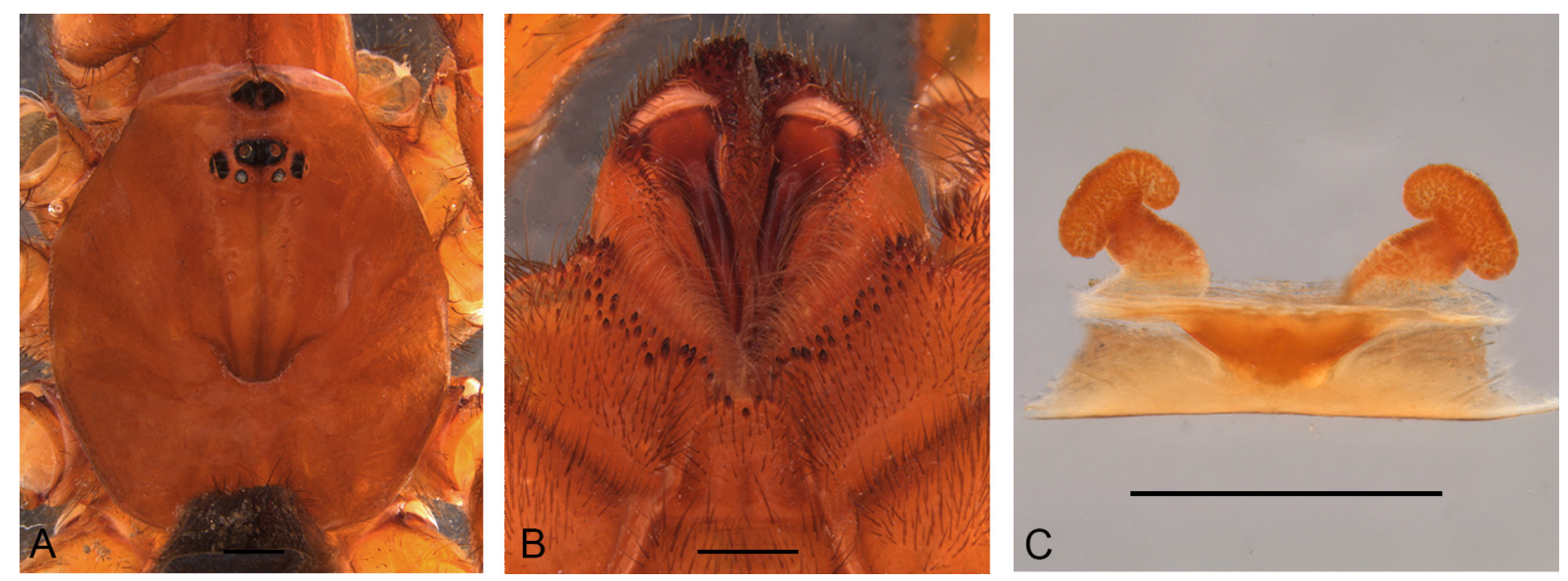

Fig. 34. Idiops siolii (Bücherl, 1953), + , neotype (MPEG 1012). A. Prosoma (dorsal view). B. Prosoma (ventral view). C. Genitalia (dorsal view). Scale bars $=1 \mathrm{~mm}$. 
half (Fig. 34B). Basal segment of chelicerae with a prolateral row of 8 large teeth and 4 small retrolateral teeth, grouped in basal half; rastellum with 20-22 short spines of same size (Fig. 34B).

Palp and leg measurements. Palp $=12.5(4.2,2.7,2.7,2.9), \mathrm{I}=13.8(4.5,2.8,3,2.1,1.4), \mathrm{II}=13.2$ $(4.3,3,2.5,2,1.4), \mathrm{III}=12(4.1,2.3,2.8,2,0.8), \mathrm{IV}=18.4(4.4,3.9,4,3.6,2.5)$.

SpinATIOn. Palp: Ti p7-9-12, r6-10-10, Ta p12-10-11, r14-12-9. Leg I: Ti p3-7-10, r9-8-10, Mt p11-8-11, r3-9-8, Ta v0-0-3, p5-4-3, r5-5-4. Leg II: Pa p5-5-12, Ti v1-1-1, p2-4-6, r0-2-3, Mt v2-2-3, d2-2-2, p64-2, r2-3-1, Ta v2-4-5, p0-2-5, r0-1-1. Leg III: Ti v1-1-0, r0-1-1, p2-6-7, Mt v2-2-4, p9-7-9, r2-2-2, Ta v0-0-3, p5-5-2, r1-0-0. Leg IV: Ti v0-0-1, Mt v0-1-2, p1-2-3, r0-1-1, Ta v2-1-2, p1-5-5, r0-2-2.

SPERMATHECAE. V-shaped ducts with sclerotized portion between duct and receptacles. Bilobed ducts sclerotized, with evident granules (Fig. 34C).

\section{Distribution}

Brazil. Known only for the region of Belém and Melgaço (Pará), in the phytophysiognomies of the Amazonian region (Fig. 3B)

\section{Species inquirenda}

\section{Idiops bonapartei Hasselt, 1888}

Idiops bonapartei Hasselt, 1888: 166.

\section{Remark}

The female holotype from Suriname is deposited in National Museum of Natural History of Leiden. It was not possible to obtain the type specimen for loan. The examination of the type through photos revealed it to be an immature female specimen of Idiopinae. Moreover, the original description does not warrant species recognition, as it includes only generic level characteristics. Because of this, we consider I. bonapartei species inquirenda.

\section{Distribution}

Suriname. Known only from the type locality (Fig. 5B).

\section{Discussion}

\section{Systematics and biogeography notes}

Trapdoor spiders belonging to the infraorder Mygalomorphae Pocock, 1892, which include the family Idiopidae, are usually associated with a sedentary lifestyle, high longevity, limited dispersion skills and high environmental specificity (Dippenaar-Schoeman 2002; Bond \& Stockman 2008; Pérez-Miles \& Perafán 2017; Mason et al. 2018). These characteristics, together with the strong morphological homogeneity contrasted with the high genetic diversification, make these spiders great evolutionary and biogeographic models (Bond et al. 2001; Satler et al. 2013; Opatova \& Arnedo 2014; Harrisson et al. 2017; Opatova et al. 2020).

The genus Idiops is the most diverse among idiopids, with 102 valid species hitherto, of which 24 occur in South America, corresponding to approximately a quarter of the known species (World Spider Catalog 2021). In this revision, an extensive number of specimens of Idiops was examined, revealing undescribed species (such as the widely distributed species I. sertania sp. nov.; Fig. 4C), indicating the existence of species with an apparently restricted distribution (such as I. nilopolensis and I. germaini) 
(Fig. 3D), and expanding the geographic distribution of several species (such as I. carajas, I. fuscus and I. pirassununguensis; Figs 3B, 4C). The latter had their distributions expanded by more than a thousand kilometers after the revision of this material.

As the only genus of Idiopinae with species distributed in the Neotropical and Paleotropical regions, with the other six genera restricted to the Paleotropical region (World Spider Catalog 2021), this revision of the Neotropical species is essential for a better evolutionary and biogeographic understanding of the genus, both in the context of deep and recent evolution and diversification. In an ancient context, it is unknown whether the species of Idiops of the Old and New World comprise a monophyletic clade. Despite the type species, Idiops fuscus, being from Brazil, previous molecular analyses included only Old World species (Hedin \& Bond 2006; Opatova et al. 2020). In an intraspecific context, we found morphologically similar widespread species, with disjunct distributions, which is poorly compatible with the sedentary habits of trapdoor spiders, suggesting the possible existence of a cryptic species complex.

The species Idiops pirassununguensis represents one of these cases: as a result of the present revision, the species had its distribution expanded to more than twice in size compared to the previous scenario, with records for phytophysiognomies in the Amazon, Caatinga and Cerrado. Presenting a conserved morphology and a continental distribution, uncommon in trapdoor spider (Buzzato et al. 2021), the current delimitation as a single species may actually be a complex of cryptic species. To solve this situation, an integrative approach is needed, involving molecular, morphometric, demographic and environmental data. Such an approach has already been taken to solve similar problems for species of the genera Aptostichus Simon, 1891 (Euctenizidae Raven, 1985) (Bond et al. 2001; Bond \& Stockman 2008), Aliatypus Smith, 1908 (Antrodiaetidae Gertsch,1940) (Satler et al. 2013), Ummidia Thorell, 1878 (Halonoproctidae Pocock, 1901) (Opatova et al. 2013, 2016), Titanidiops (Idiopidae) (Opatova \& Arnedo 2014) and Moggridgea O. Pickard-Cambridge, 1875 (Migidae Simon,1889) (Harrisson et al. 2017).

An alternative hypothesis that may explain such a wide distribution that cannot be ruled out is ballooning, which is a behavioral mechanism of aerial dispersion with the use of silk, widely reported for silkproducing arthropods such as spiders, mites and moth larvae (Bell et al. 2005). Although not commonly witnessed in Mygalomorphae (Colye 1983; Bell et al. 2005; Buzzato et al. 2021), as in Araneomorphae Pocock, 1892, cases of ballooning dispersion have already been recorded for at least nine species from four families, all of trapdoor spiders (Atypidae Thorell, 1870, Actinopodidae Simon,1892, Halonoproctidae and recently Idiopidae) (Buzzato et al. 2021; Rossi et al. 2021). In this type of aerial dispersion, called suspended ballooning, spiderlings climb to elevated positions in the vegetation, exposing themselves to be carried passively by the wind, with the help of a drag line (Colye 1983; Bell et al. 2005; Buzzato et al. 2021). According to Opatova et al. (2013), ballooning possibly contributed to the dispersion of spiders of the genus Ummidia across the Mediterranean and between Europe and North America, when the continents were not too far apart. The ballooning, which has already been recorded for several species of Ummidia, could also explain the presence of the genus on Caribbean islands (Opatova et al. 2013; Godwin \& Bond 2021).

For Idiopidae, ballooning was recently recorded for the species Neocteniza toba Goloboff, 1987 (Genysinae), a widely distributed trapdoor spider with records in Argentina, Brazil and Paraguay (Rossi et al. 2021). From laboratory experiments, the authors observed spiderlings being carried by gentle breezes reaching 20-30 cm from the dropping point, with some individuals reaching a distance of three meters from the initial point (Rossi et al. 2021). Although not directly registered, ballooning dispersion was inferred for species of the subfamily Arbanitinae with wide distribution, found in Australia (Buzzato et al. 2021). To explore the importance of ballooning in the distribution of Neotropical Idiops, such as I. pirassununguensis, we need studies that can infer, or even directly record, ballooning and investigate 
population genetic structuring, to estimate spiderling dispersal skills from their maternal burrows (Buzzato et al. 2021).

\section{Acknowledgements}

We wish to give our thanks to all curators for the loan of specimens, to Beatriz Maurício, for her help with SEM images, to Abel Pérez Gonzáles and John Kochalka for sending the male of I. rohdei, to our arachnologist friends Arthur Galetti Lima, David Candiani, Felipe Garcia, Hector Gonzales Filho, Júlia Ayumi Ando, Leandro Malta Borges, Leonardo Souza Carvalho, Millke Jasmini Morales, Pedro Henrique Martins, Rafael Prezzi Indicatti, Willian Fabiano Silva and Wolf Moeller for their assistance and critical suggestions for this work, and to Leonardo Carvalho, Pedro Henrique Martins and Rafael Prezzi Indicatti for sending us photos. We also thank to the reviewers for their comments and suggestions that improved our manuscript. This study was financed by the Fundação de Amparo à Pesquisa do Estado de São Paulo (FAPESP 2018/02842-2 to RFF) (FAPESP 2017/11985-9 to JPLG) (FAPESP 2008/10147-0 to FUY) and CNPq $(303903 / 2019-8$ to ADB).

\section{References}

Ausserer A. 1875. Zweiter Beitrag zur Kenntniss der Arachniden-Familie der Territelariae Thorell (Mygalidae Autor). Verhandlungen der Kaiserlich-Königlichen Zoologisch-Botanischen Gesellschaft in Wien 25: 125-206.

Bell J.R., Bohan D.A., Shaw E.M. \& Weyman G.S. 2005. Ballooning dispersal using silk: world fauna, phylogenies, genetics and models. Bulletin of Entomological Research 95 (2): 69-114. https://doi.org/10.1079/BER2004350

Bond J.E. \& Stockman A.K. 2008. An integrative method for delimiting cohesion species: finding the population-species interface in a group of Californian trapdoor spiders with extreme genetic divergence and geographic structuring. Systematic Biology 57 (4): 628-646. https://doi.org/10.1080/10635150802302443

Bond J.E., Hedin M.C., Ramirez M.G. \& Opell B.D. 2001. Deep molecular divergence in the absence of morphological and ecological change in the Californian coastal dune endemic trapdoor spider Aptostichus simus. Molecular Ecology 10 (4): 899-910. https://doi.org/10.1046/j.1365-294X.2001.01233.x

Bond J.E., Hendrixson B.E., Hamilton C.A. \& Hedin M. 2012. A reconsideration of the classification of the spider infraorder Mygalomorphae (Arachnida: Araneae) based on three nuclear genes and morphology. PloS One 7 (6): e38753. https://doi.org/10.1371/journal.pone.0038753

Buzatto B.A., Haeusler L. \& Tamang N. 2021. Trapped indoors? Long-distance dispersal in mygalomorph spiders and its effect on species ranges. Journal of Comparative Physiology A 207: 279-292. https://doi.org/10.1007/s00359-020-01459-x

Bücherl W. 1957. Sôbre a importância dos bulbos copuladores e das apófises tibiais dos machos na sistemática das aranhas caranguejeiras (Orthognatha). Anais da Academia Brasileira de Ciências 29: $377-416$.

Bücherl W., Timotheo da Costa A. \& Lucas S. 1971. Revisão de alguns tipos de aranhas caranguejeiras (Orthognatha) estabelecidos por Cândido de Mello-Leitão e depositados no Museu Nacional do Rio. Memórias do Instituto Butantan 35: 117-138.

Coyle F.A. 1983. Aerial dispersal by mygalomorph spiderlings (Araneae, Mygalomorphae). Journal of Arachnology 11 (2): 283-286.

Coyle F.A., Dellinger R.E. \& Bennet R. 1992. Retreat architecture and construction behavior of an East African idiopine trapdoor spider (Araneae, Idiopidae). Bulletin of the British Arachnological Society 9: 99-104. 
Dippenaar-Schoeman A.S. 2002. Baboon and Trapdoor Spiders of Southern Africa: An Identification Manual. Plant Protection Research Institute Handbook No. 13. Agricultural Research Council, Pretoria.

Dupérré N. \& Tapia E. 2021. The endless search for type specimens; illustrations of eleven spider (Araneae, Mygalomorphae) species described by Eugène Simon. Zootaxa 4951 (2): 259-282. https://doi.org/10.11646/zootaxa.4951.2.3

Ferretti N., Nime M. \& Mattoni C. 2017. Three new Idiops (Mygalomorphae: Idiopidae) from Argentina and redescription of the male of I. hirsutipedis Mello-Leitão, 1941. Journal of Natural History 51 (17-18): 975-994. https://doi.org/10.1080/00222933.2017.1319516

Fonseca-Ferreira R., de Almeida Zampaulo R. \& Guadanucci J.P.L. 2017. Diversity of iron cave-dwelling mygalomorph spiders from Pará, Brazil, with description of three new species (Araneae). Tropical Zoology 30 (4): 178-199. https://doi.org/10.1080/03946975.2017.1367590

Fukami C.Y. \& Lucas S.M. 2005. Notas sobre espécies de Idiops Perty, 1833 do sudeste Brasileiro com descrição de I. pirassununguensis sp. nov. (Mygalomorphae, Idiopidae, Idiopinae). Biota Neotropica 5 (18051a): 1-7. https://doi.org/10.1590/S1676-06032005000200016

Godwin R.L. \& Bond J.E. 2021. Taxonomic revision of the New World members of the trapdoor spider genus Ummidia Thorell (Araneae, Mygalomorphae, Halonoproctidae). ZooKeys 1027: 1-165. https://doi.org/10.3897/zookeys.1027.54888

Goloboff P.A. 1993. A reanalysis of mygalomorph spider families (Araneae). American Museum Novitates 3056:1-32.

Guérin F.E. 1838. Arachnides. In: Eydoux J.F.T. \& P. Gervais (eds) Voyage autour du monde par les mers de l'Inde et de Chine exécuté sur la corvette de l'Etat La Favorite pendant les années 1830/1831 et 1832 sous le commandement de M. Laplace capitaine de frégate: 5, 161-167, pl. 47-48. Paris.

Gupta N., Ganeshkumar M., Das S.K. \& Siliwal M. 2013. Three new species of Idiops Perty, 1833 (Araneae: Idiopidae) from India. Zootaxa 3635 (3): 237-250. https://doi.org/10.11646/zootaxa.3635.3.3

Gupta N., Das S.K. \& Siliwal M. 2015. Natural history of the trapdoor spider Idiops joida Gupta et al 2013 (Araneae: Idiopidae) from the Western Ghats in India. Journal of Asia-Pacific Biodiversity 8 (1): 38-42. https://doi.org/10.1016/j.japb.2015.01.004

Harrison S.E., Harvey M.S., Cooper S.J., Austin A.D. \& Rix M.G. 2017. Across the Indian Ocean: a remarkable example of trans-oceanic dispersal in an austral mygalomorph spider. PloS One 12 (8): e180139. https://doi.org/10.1371/journal.pone.0180139

Hasselt A.W.M. van. 1888. Araneae exoticae quas collegit, pro Museo Lugdunensi, Dr H. Ten Kate Jr. in Guyanâ Hollandicâ (Suriname). Tijdschrift voor Entomologie 31: 165-200.

Hedin M. \& Bond J.E. 2006. Molecular phylogenetics of the spider infraorder Mygalomorphae using nuclear rRNA genes (18S and 28S): conflict and agreement with the current system of classification. Molecular Phylogenetics and Evolution 41 (2): 454-471. https://doi.org/10.1016/j.ympev.2006.05.017

ICZN. 1999. International Code of Zoological Nomenclature. Fourth Edition. International Trust for Zoological Nomenclature, London, UK. Available from https://www.iczn.org/the-code [accessed 28 Jul. 2021].

Karsch F. 1886. Araneologisches aus Südamerika. Berliner Entomologische Zeitschrift 30: 92-94.

Mason L.D., Wardell-Johnson G. \& Main B.Y. 2018. The longest-lived spider: mygalomorphs dig deep, and persevere. Pacific Conservation Biology 24 (2): 203-206. https://doi.org/10.1071/PC18015.

Mello-Leitão C.F. 1923. Theraphosideas do Brasil. Revista do Museu Paulista 13: 1-438. 
Mello-Leitão C.F. 1930. Aranhas do Cuminá. Arquivos do Museu Nacional do Rio de Janeiro 32: 51-75. Mello-Leitão C.F. 1937. Aranhas novas ou raras. Anais da Academia Brasileira de Ciências 9: 1-12.

Mello-Leitão C.F. 1946. Nuevos aracnidos sudamericanos de las colecciones del Museo de Historia Natural de Montevideo. Comunicaciones Zoologicas del Museo de Historia Natural de Montevideo 2 (35): $1-10$.

Mirza Z.A. \& Sanap R.V. 2012. A new species of the genus Idiops and notes on Idiops bombayensis Siliwal et al. 2005 (Araneae: Idiopidae) from Northern Western Ghats of Maharashtra, India. The Journal of Arachnology 40 (1): 85-95. https://doi.org/10.1636/A11-37.1

Mirza Z.A., Vaze V.V. \& Sanap R.V. 2012. A new species of the trapdoor spiders genus Idiops Perty, 1833 (Araneae: Idiopidae) from the Western Ghats, with a key to the Idiops of India. Revista Ibérica de Aracnología 21: 9-14.

Moreira T.D.S., Baptista R.L., Kury A.B., Giupponi A.P., Buckup E.H. \& Brescovit A.D. 2010. Annotated check list of Arachnida type specimens deposited in the Museu Nacional, Rio de Janeiro. II - Araneae. Zootaxa 2588 (1): 1-91. https://doi.org/10.11646/zootaxa.2588.1.1

Opatova V. \& Arnedo M.A. 2014. Spiders on a hot volcanic roof: colonisation pathways and phylogeography of the Canary Islands endemic trap-door spider Titanidiops canariensis (Araneae, Idiopidae). PLoS One 9 (12): e115078. https://doi.org/10.1371/journal.pone.0115078

Opatova V., Bond J.E. \& Arnedo M.A. 2013. Ancient origins of the Mediterranean trap-door spiders of the family Ctenizidae (Araneae, Mygalomorphae). Molecular Phylogenetics and Evolution 69 (3): 1135-1145. https://doi.org/10.1016/j.ympev.2013.08.002

Opatova V., Bond J.E. \& Arnedo M.A. 2016. Uncovering the role of the Western Mediterranean tectonics in shaping the diversity and distribution of the trap-door spider genus Ummidia (Araneae, Ctenizidae). Journal of Biogeography 43 (10): 1955-1966. https://doi.org/10.1111/jbi.12838

Opatova V., Hamilton C.A., Hedin M., De Oca L.M., Král J. \& Bond J.E. 2020. Phylogenetic systematics and evolution of the spider infraorder Mygalomorphae using genomic scale data. Systematic Biology 69 (4): 671-707. https://doi.org/10.1093/sysbio/syz064

Pérez-Miles F. \& Perafán C. 2017. Behavior and biology of Mygalomorphae. In: Viera C. \& Gonzaga M.O. (eds) Behaviour and Ecology of Spiders. Springer International Publishing, Cham, Switzerland. https://doi.org/10.1007/978-3-319-65717-2

Perty M. 1833. Arachnides Brasilienses. In: de Spix J.B. \& Martius F.P. (eds) Delectus animalium articulatorum quae in itinere per Braziliam ann. 1817 et 1820 colligerunt: 191-209, pls 38-39. Munich.

Petrunkevitch A. 1911. A synonymic index-catalogue of spiders of North, Central and South America with all adjacent islands, Greenland, Bermuda, West Indies, Terra del Fuego, Galapagos, etc. Bulletin of the American Museum of Natural History 29: 1-791. https://doi.org/10.5962/bhl.title.23819

Petrunkevitch A. 1925. Arachnida from Panama. Transactions of the Connecticut Academy of Arts and Sciences 27: 51-248.

Pickard-Cambridge O. 1870. Monograph of the genus Idiops, including descriptions of several species new to science. Proceedings of the Zoological Society of London 1870: 101-108. Available from https://www.biodiversitylibrary.org/part/67316 [accessed 2 Nov. 2021].

Pickard-Cambridge O. 1889. On a new tree trap-door spider from Brazil. Proceedings of the Zoological Society of London 1889: 250-252.

Pickard-Cambridge F.O. 1896. On the Theraphosidae of the lower Amazons: being an account of the new genera and species of this group of spiders discovered during the expedition of the steamship 
"Faraday" up the river Amazons. Proceedings of the Zoological Society of London 64 (3): 716-766. https://doi.org/10.1111/j.1096-3642.1896.tb03076.x

Pocock R.I. 1837. On the spiders of the suborder Mygalomorphae from the Ethiopian Region, contained in the collection of the British Museum. Proceedings of the Zoological Society of London 65 (3): 724-774. https://doi.org/10.1111/j.1096-3642.1897.tb03116.x

Pocock R.I. 1893. Description of a new species of tree trap-door spider from Trinidad. Annals and Magazine of Natural History (6) 11: 407-409. https://doi.org/10.1080/00222939308677548

Pocock R.I. 1895. Notes on the identity of some of the types of Mygalomorphae in the collection of the British Museum. Journal of Natural History 16 (93): 223-230. https://doi.org/10.1080/00222939508680262

Raven R.J. 1985. The spider infraorder Mygalomorphae (Araneae): cladistics and systematics. Bulletin of the American Museum of Natural History 182: 1-180.

Rix M.G., Cooper S.J., Meusemann K., Klopfstein S., Harrison S.E., Harvey M.S. \& Austin A.D. 2017 a. Post-Eocene climate change across continental Australia and the diversification of Australasian spiny trapdoor spiders (Idiopidae: Arbanitinae). Molecular Phylogenetics and Evolution 109: 302-320.

https://doi.org/10.1016/j.ympev.2017.01.008

Rix M.G., Raven R.J., Main B.Y., Harrison S.E., Austin A.D., Cooper S.J. \& Harvey M.S. 2017b. The Australasian spiny trapdoor spiders of the family Idiopidae (Mygalomorphae: Arbanitinae): a relimitation and revision at the generic level. Invertebrate Systematics 31 (5): 566-634. https://doi.org/10.1071/IS16065

Roewer C.F. 1953. Araneae-Orthognatha (Arachnoidae). Exploration du Parc National de l'Upemba, Mission G. F. de Witte 22: 1-80.

Rossi G.F., Ghirotto V.M., Galleti-Lima A., Indicatti R.P. \& Guadanucci J.P.L. 2021. "Flying” or digging? The trapdoor spider genus Neocteniza Pocock, 1895: redescription of three species, new records from Brazil, notes on natural history and first record of ballooning for Idiopidae (Araneae, Mygalomorphae). Zootaxa 5023 (4): 451-485. https://doi.org/10.11646/zootaxa.5023.4.1

Satler J.D., Carstens B.C. \& Hedin M. 2013. Multilocus species delimitation in a complex of morphologically conserved trapdoor spiders (Mygalomorphae, Antrodiaetidae, Aliatypus). Systematic Biology 62 (6): 805-823. https://doi.org/10.1093/sysbio/syt041

Schiapelli R.D. \& Gerschman de Pikelin B.S. 1971. Estudio de algunas arañas descriptas por Mello-Leitão para el Uruguay. Revista de la Sociedad Entomológica Argentina 33 (1-4): 57-62.

Siliwal M., Hippargi R., Yadav A. \& Kumar D. 2020. Five new species of trap-door spiders (Araneae: Mygalomorphae: Idiopidae) from India. Journal of Threatened Taxa 12 (13): 16775-16794. https://doi.org/10.11609/jott.5434.12.13.16775-16794

Simon E. 1889. Voyage de M. E. Simon au Venezuela (Décembre 1887-Avril 1888). 4e Mémoire. Arachnides. Annales de la Société Entomologique de France 9 (6): 169-220.

Simon E. 1892. Histoire naturelle des Araignées. Deuxième édition, Tome premier, No 1. Roret, Paris. https://doi.org/10.5962/bhl.title.51973

Simon E. 1903. Histoire naturelle des Araignées. Deuxième édition, Tome second, No 4. Roret, Paris. https://doi.org/10.5962/bhl.title.51973

Soares B.A.M. 1944. Aracnideos de Monte Alegre. Papéis Avulsos do Departamento de Zoologia, Secretaria de Agricultura, Sao Paolo 4: 151-168.

Tucker R.W.E. 1917. On some South African Aviculariidae (Arachnida). Families Migidae, Ctenizidae, Diplotheleae and Dipluridae. Annals of the South African Museum 17 (2): 79-138. 
Walckenaer C.A. 1837. Histoire naturelle des insectes. Aptères. Tome premier, No. 2. Roret, Paris. https://doi.org/10.5962/bhl.title.61095

Wilson J.D., Hughes J.M., Raven R.J., Rix M.G. \& Schmidt D.J. 2018. Spiny trapdoor spiders (Euoplos) of eastern Australia: broadly sympatric clades are differentiated by burrow architecture and male morphology. Molecular Phylogenetics and Evolution 122: 157-165. https://doi.org/10.1016/j.ympev.2018.01.022

Wilson J.D., Raven R.J., Schmidt D.J., Hughes J.M. \& Rix M.G. 2020. Total-evidence analysis of an undescribed fauna: resolving the evolution and classification of Australia's golden trapdoor spiders (Idiopidae: Arbanitinae: Euoplini). Cladistics 36 (6): 543-568. https://doi.org/10.1111/cla.12415

World Spider Catalog. 2021. World Spider Catalog. Version 21.5. Natural History Museum Bern. https://doi.org/10.24436/2

Manuscript received: 28 April 2021

Manuscript accepted: 7 October 2021

Published on: 25 November 2021

Topic editor: Rudy C.A.M. Jocqué

Desk editor: Radka Rosenbaumová

Printed versions of all papers are also deposited in the libraries of the institutes that are members of the EJT consortium: Muséum national d'histoire naturelle, Paris, France; Meise Botanic Garden, Belgium; Royal Museum for Central Africa, Tervuren, Belgium; Royal Belgian Institute of Natural Sciences, Brussels, Belgium; Natural History Museum of Denmark, Copenhagen, Denmark; Naturalis Biodiversity Center, Leiden, the Netherlands; Museo Nacional de Ciencias Naturales-CSIC, Madrid, Spain; Real Jardín Botánico de Madrid CSIC, Spain; Zoological Research Museum Alexander Koenig, Bonn, Germany; National Museum, Prague, Czech Republic. 